\title{
A Shielding Calculational System for Plutonium
}

by

M. G. Zimmerman and

D. H. Thomsen

August 1975

Prepared for the U.S. Energy

Research and Development Administration under Contract E(45-1):1830 
NOTICE

This report was prepared as an account of work sponsored by the United States Government. Neither the United States nor the United States Energy Research and Development Administration, nor any of their emplovees. nor any of their contractors, subcontractors, or their employees, makes any warranty, express or implied, or assumes any legal liability or responsibility for the accuracy, completeness or usefulness of any information. apparatus, product or process disclosed, or represents that its use would not infringe privately owned rights.

\author{
PACIFIC NORTHWEST LABORATORY \\ operated by \\ BATTELLE \\ for the
}

U.S. ENERGY RESEARCH AND DEVELOPMENT ADMINISTRATION

Under Contract $E(45-1)-1830$

Printed in the United States of America

Available from

National Technical Information Service

U.S. Department of Commerce

5285 Port Royal Road

Springtield, Virginia 22151

Price: Printed Copy \$5.45; Microfiche \$2.25 
BNWL -1855

UC -32

Special

Distribution

A SHIELDING CALCULATIONAL SYSTEM FOR PLUTONIUM

by

M. G. Zimmerman and

D. H. Thomsen

August 1975

Battelle

Pacific Northwest Laboratories

Richland, Washington 99352 


\section{ACKNOWLEDGMENTS}

The project was initiated by the Advanced Operational Planning Group at Hanford. Further support for the proposed objectives was obtained from the AECOP at Oak Ridge. The support and advice of J. M. Morrison of AECOP and A. E. Smith of ARHCO were especially helpful. L. G. Faust and L. W. Brackenbush of PNL performed the experimental measurements and were especially helpful in making available experimental results from other AEC programs. Aid in obtaining and evaluating nuclear data was provided by D. A. Kottwitz, J. L. Carter, and D. L. Lessor, all of PNL. Further thanks are due for the advice and support of H. H. Van Tuyl of PNL and also with J. N. Strode and R. C. Smith of HEDL for helping make the code PUSHLD and experimental data available for this project. Useful suggestions and support were also supplied by E. A. Putzier and D. C. Coonfield of Rocky Flats. Personnel at AEC headquarters such as F. X. Murray have also been helpful with comments and suggestions. N. E. Carter was instrumental in getting the project completed because of his advice and encouragement. 


\section{ABSTRACT}

A computer calculational system has been developed and assembled specifically for calculating dose rates in AEC plutonium fabrication facilities. The system consists of two comnuter codes and all nuclear data necessary for calculation of neutron and gamma dose rates from plutonium. The codes include the multigroup version of the Battelle Monte Carlo code for solution of general neutron and gamma shielding problems and the PUSHLD code for solution of shielding problems where low energy gamma and $x$-rays are important. The nuclear data consists of built in neutron and gamma yields and spectra for various plutonium compounds, an automatic calculation of age effects and all cross-sections commonly used. Experimental correlations have been performed to verify portions of the calculational system. 
ACKNOH EDGMENTS

ACKNOWLEDGMENTS. . . . . . . . . . . . . . . . $i$

ABSTRACT . . . . . . . . . . . . . . . . . . 1 . 1 .

LIST OF TABLES . . . . . . . . . . . . . . . v v

LIST OF FIGURES ............................ vij

I. INTRODUCTION ...................... 1

II. SUMMARY AND RECOMMENDATIONS. . . . . . . . . . . 3

III. TECHNICAL APPROACH ................ 5

IV. NUCLEAR DATA . . . . . . . . . . . . . . 9

V. CALCULATIONS AND CORRELATIONS. . . . . . . . . 12

VI. REFERENCES ................. 16

APPENDIX A: DESCRIPTION OF THE MULTIGROUP VERSION OF THE BATTELLE MONTE CARLO COMPUTER CODE

APPENDIX B: DESCRIPTION OF THE PUSHLD COMPUTER CODE

APPENDIX C: DESCRIPTION OF A SIMPLE NEUTRON-GAMMA CROSS-SECTION COUPLING COMPUTER CODE 


\section{LIST OF TABLES}

Table

Page No.

1 Neutron Energy Group Structure Used Ey System. . . . . 18

227 Group Neutron Cross-Sections In System Library. . . . 19

3 Gamma Energy Group Structure Used By System. . . . . . 20

416 Group Gamma Cross-Section In System Library . . . . 21

5 Gamma Production Cross-Sections Available in System

Library...................... 22

6 Neutron Yields In Neutrons/Sec-gram of Pu. . . . . 23

7 Neutron Source Spectra Built Into System . . . . . . 24

8 Gamma Spectra And Yields By Energy Group . . . . . . 25

9 Ratio of Calculated Kerma To Experimental Kerma. . . . . 26

10 Isotopic Plutonium Composition for Source in Neutron Dose Rate Measurement Experiment. . . . . . . . . 26

11 Comparison of Calculations To Experimental Measurements of Neutron Kerma Rate From A Glove Box . . . . . . 27

i2 Comparison of Calculations To Experiment For Rocky Flats And Burlington Measurements............ 28

13 Plutonium Isotonic Composition For Rocky Flats And Burlington Measurements...............

14 a Activity From Am-241 As A Function of Plutonium Age For Plutonium Composition of Table 13........ 29

15 Initial Pu Isotopic Composition For Gamma Dose Rate Measurements............... 30

16 Comparison of Experimental Measurements And Calculational Gama Dose Rates From $\mathrm{PuO}_{2}$. . . . . . . . . . . 31

17 Source Descriptions For AECOP. . . . . . . . . 32

18 Shielding Cases For AECOP. . . . . . . . . . 33

19 Gama Dose Rate Calculational Result; For AECOP. . . . 34 
20 Gamma Dose Rate Calculational Results For AECOP. . . . 35

21 Gamma Dose Rate Calculational Results For AECOP. . . . 36

22 Calculated Neutron Dose Rates For AECOP. . . . . . 37

23 Plutonium Metal Isotopic Compositions Used For AECOP

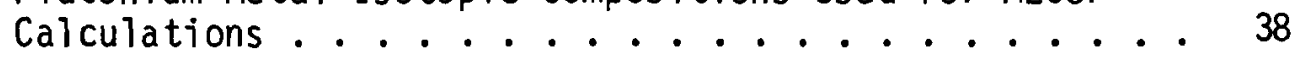




\section{LIST OF FIGURES}

Figure Page

1 The Shielding Calculational System For Plutonium. . . 39

2 Plutonium Isotopic Decay Schemes. . . . . . . . 40

3 View of Plutonium Hood In Configuration Used For

Experimental Dose Rate Measurements . . . . . . . 41

4 Glove Box Line As Modeled By The BMC-MG Code. . . . 42

5 Section of Glove Box Line As Modeled By The BMC-MG Code................... 43

6 Neutron Dose Rate Versus Distance From Source In A Glove Box Line. ............... 44

7 Gamma Dose Rate Measurement Experiment. . . . . . . 45 
Introduction

This report describes a project undertaken to satisfy the U. S. Atomic Energy Commission's need for a calculational system capable of predicting radiation dose rates from plutonium and its daughter products. The system as developed can predict dose rates as a function of age, mass, geometry, isotopic composition and shielding configuration. The calculational system was being developed primarily for use in the analytical study, "Combined Plutonium Production and Processing Operations." However, the system also has potential for continuing application in other AEC activities associated with plutonium processing.

An important use of a calculation system is in the realm of safety. The system can facilitate the prediction of safety problems before they occur and can contribute to better management of personnel dose by predicting changes in personnel dose due to facility and processing changes. Overall, the analytical capability provided by the system will permit the evaluation of many more operational and facility alternatives than were previously possible.

Some savings in exposure or dollars might be realized through use of the system to generate better predictions of dose rates and shielding requirements. These predictions would allow a more precise determination of the optimum balance between building costs and operating costs of plutonium processing facilities. In the design of new and modified facilities, considerable reliance is placed on previous experience with similar facilities together with all available experimental evidence. This computer system could be used to augment these design efforts by providing a means of identifying potential problem areas early in the design stages. Also, experimental evidence can be used to verify proper modeling by the system and, the system can then be used to perform parametric studies and consider many more alternatives than could easily be performed experimentally. This advantage applies likewise to the analysis of existing and possible alternative operations. 
Basically, the calculational system includes the computer codes and necessary nuclear data needed to calculate dose rates from the following sources:

1. Spontaneous fission neutrons.

2. Induced fission neutrons.

3. $(\alpha, n)$ neutrons.

4. Primary gamma rays.

5. Secondary gamma rays $(n, \gamma)$.

The computational system consists of a general geometry-threedimensional Monte Carlo code, a nuclear data manipulation code, necessary nuclear data libraries, and a special code developed to calculate plutonium gamma and $X$-ray dose rates through very thin shields such as gloves. The nuclear data include sources and spectra from both fission and radioactive decay for the plutonium isotopes and daughters. Also included are spectra and yields for $(\alpha, n)$ reactions and for several plutonium compounds.

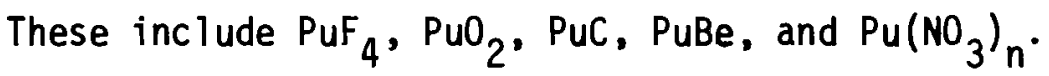


Summary and Recommendations

A computer calculational system has been developed and verified experimentally as being capable of calculating neutron and gamma dose rates from shielded plutonium sources as a function of plutonium mass, isotopic composition, and age as a function of source and shield geometry. The system makes use of a three-dimensional multigroup Monte Carlo code (BMC), a point kernel code specially designed for low energy gammas and $X$-rays through thin shields (PUSHLD) and the requisite nuclear data.

Experimental-calculational studies were performed to verify the neutron and gamma dose rate calculational capability of the system. These studies were reasonably successful. However, the $(n, y)$ calculation portion has not been verified experimentally except by reference to the work of others using similar data. Verification of this portion should be performed.

Recentlv a disagreement was discovered between the qamma dose rates that the PUSHLD ${ }^{(1)}$ code predicts and what personnel at Rocky Flats expect from hand calculations. For a large plutonium metal source shielded by a $30 \mathrm{mil}$ leaded glove, PUSHLD indicates a lower proportion of the total dose due to ${ }^{241} \mathrm{Am}$ than Rocky Flats obtains. The behavior of the ${ }^{241} \mathrm{Am}$ dose rate with time is the same in both cases. Further efforts should be made to resolve the reasons for this.

Some additions to the Monte Carlo code which are probably desirable were not added because of lack of time and funds. This includes a diffusion kernel for handling the thermal neutrons, which would reduce computer time. Also, a more efficient way of handling the cross-sections to allow more materials to be used in coupled neutron-gamma calculations would be useful. There are several other smaller additions which would add to convenience in using the system.

Also, the nuclear data should be checked and replaced as new and better data become available. This is expecially true in regard to $(n, \gamma)$ source data and to a lesser but significant extent for primary gamma yields and spectra. Perhaps nuclear data for more isotopes would be desirable in the future, too. 
The PUSHLD portion of the system is at this time 1 imited by the different types of shield materials built into the code. This is due to the seeming lack of data on low energy buildup factors. Conceivably these low energy bulldup factors could be developed analytically. More shielding matertals would add to the flexibility of the PUSHLD code.

The system is now operational and available to those desirina it. At present a CYBER 74 version is avallable. The code will be sent to the Radiation Shielding Information Center at Oak Ridge. The components available with this document include:

1. Battelle Monte Carlo Code

2. PUSHLD Code

3. Neutron cross-sections

4. Gamma cross-sections

5. Neutron-gamma production cross-sections

6. Code to combine cross-sections into coupled neutron-gamma cross-sections

7. Sample problem input and output for the codes. 
Terhnira 1 Annmarh

Determining the technical approach to the problem of calculating dose rates in plutonium processing facilities requires consideration of the types of problems expected, the accuracy desired, ease of operation, and computer time economy.

Upon examination of the types of problems expected, it becomes apparent that a wide range of problem types might need solution in the facility. These range from vaults containing large aillouints of $\mathrm{Pu}$ to glove boxes containing relatively small amounts of $\mathrm{Pu}$ or a series of glove boxes containing a variety of $\mathrm{Pu}$ sources. Other examples of possible complicated geometries are dissolver columns and pipes containing solutions. Dose rates to specific parts of the body might be required such as to hands and whole body. In many cases a very significant proportion of the dose rate will be contributed by source multiplication of the neutrons and by scatter around shields. Very thin shields such as gloves must be considered as well as several foot thicknesses of concrete.

Another requirement is that the system should be usable with confidence. This means that assumptions to be made in order to calculate a problem should be kept to a reasonable minimum. Trying to obtain reasonable accuracy in a system which requires gross assumptions as to shield geometry, scatter, or multiplication would seem to be self-defeating. In such a system, the possible reasons for large disagreement with experimental results or experience would of necessity be ill-defined.

From the foregoing, a list of requirements for a computer code system can be developed. First of all the code system must be able to calculate source multiplication of neutrons with sufficient accuracy to determine reasonably accurate neutron dose rates. This seems fairly obvious since in many cases the induced fission neutrons can easily contribute a major share of the dose rate.

The next requirement is that the system be able to handle three dimensional shielding and source geometries. There are several good reasons for this. As pointed out above the source multiplication can be an important part of the dose rate calculation. To accurately calculate 
the multiplication for the shapes of sources expected requires that the sources be modeled in three dimensions. Such sources include shells, discs, cylinders and others of various densities and dimensions. The multiplication effects would vary greatly among these sizes, shapes, and densities. Another reason for the three dimensional capability is that shield geometry is often such that there are streaming voids such as glove ports and it is often quite important to know the amount of scattering around the shields from walls, floors etc. This would be difficult to model in less than three dimensions. If two dimensions were used, questionable assumptions would be necessary and in one dimension, gross assumptions would be necessary. This should not be construed as indicating that one and two dimensional codes are not completely adequate for many problems. However, for most of the situations covered it is preferable to be able to model the geometry in three dimensions and it is often necessary in order to obtain accurate results. Therefore, provided other penalties due to complexity and computer time are not over-riding, a three dimensionai code is the proper choice.

Of the types of radiation transport codes available, three were seriously considered. These were point kernel, discrete ordinates, and Monte Carlo. Of these codes only Monte Carlo and point kernel codes are available in practical three dimensional versions. Because of the problem of modeling realistic geometries and calculating scattering contributions with the one and two dimensional discrete ordinates codes, they were not utilized. Also, for some of the types of problems which need to be solved, the computer core area needed for two-dimensional discrete ordinate codes is larger then that availabie on many of the widely used computers.

The point kernel type of code was not chosen for several reasons. Some point kernel codes 11ke QADP5A are avallable which calculate neutron transport. However, there is probably no practical way to calculate neutron multiplication with the code and the code at present cannot calculate scattering of neutrons from floors, walls, etc. around shields. The code also lacks a methodology for calculating the $(n, \gamma)$ contribution. The code can calculate primary 
gamma transport, but even here another way to handle buildup factors of laminated shields is needed such as presented in reference $2 .{ }^{(2)}$ For very low energy gammas and $x$-rays shielded by shields such as gloves, the buildup factor and attenuation factor methodology is not adequate.

The three dimensional, general geometry, multigroup Monte Carlo(3) code was chosen. The code is able to realistically model practically any of the problems which will be encountered. The code can calculate neutron transport, primary gamma transport, neutron multiplication, and secondary gamma production and transport in a single calculation for a particular problem. The code will also calculate scattering off of walls, floors etc. This type of code is capable of doing most of the calculations necessary for plutonium fabrication facilities. Even so, any choice is a compromise.

For simple problems, the Monte Carlo program can be easily set up and run to produce calculated dose rates in reasonably short computer times. The set up is straight forward and simple. By a simple problem is meant a source of given size and shape shielded by several shields less than about several mean free paths thick and including source multiplication and secondary gammas. For more complex problems the setup time is longer and more tedious and not so straight forward. Even for complex problems, setting up the geometry, detectors, and sources is straight forward, if tedious. However, for complex problems where scattering off of walls is important and with thick shields of several feet of hydrogenous material, computer times can be unrealistic, especially if various options are not used correctly. These options are the biasing and importance weighting options described in the code description section. To some extent the proper use of these options must be determined through experience.

Another possible problem in using the Monte Carlo code is confusion over interpretation of the statistical errors printed out with the results. These are errors for one sigma deviation from the mean. The size of these statistical errors bears directly on the combuter running times since they are indirectly proportional to the square root of the number of particles followed by the code. Hence to decrease the statistical error from 10\% to $1 \%$ would require an increase in running time of approximately 100 . The reason the code is viable for plutonium facility shielding problems is that 
low statistical errors are not generally needed. Generally, we consider our calculations adequate at $10 \%$ statistical error. Depending on the uses to be made of the results of the problem, even larger statistical errors may be tolerated.

Another code is also part of this system. The Monte Carlo code and $i$ ts associated nuclear data file does not adequately calculate very low energy gamma and $x$-ray transport through thin shields such as gloves. Instead a code which was developed under another AEC program has been provided. This is a point kernel code called PUSHLD $(1)$ developed specifically to calculate dose rates from these low energy photon radiations. Basically the major improvement over previous point kernel codes is a more precise interpolation of attenuation factors and buildup factors as a function of energy. The PUSHLD code is limited both as to geometries allowed and the number of materials provided. This is due to the lack of appropriate buildup and attentuation factors at these low energies, approximately $10 \mathrm{kev}$ to $100 \mathrm{kev}$. For relatively thick shields for which these low energy radiations are relatively unimportant, the Monte Carlo code can be used, although PUSHLD is not limited to thin shields.

The calculational system is displayed in Figure 1 . The components above the dotted line are used to produce cross-section data and are described in the following section. The portion below the line is calculational system to be reieased for aistribution. This consists of the following:

1. Battelle Multi-Group Monte Carlo Code (PMC-MG)

2. PUSHLD Code

3. Neutron Cross-Sections

4. Gamma Cross-Sections

5. Neutron-Gamma Production Cross-Sections

6. Code to Combine Cross Sections Into a Coupled Neutron-Gamma Cross-Section

7. Sample Problem Input and Output 
Nuclear Data

The nuclear data needed for the system consists of the following items:

1. Neutron cross-sections

2. Gamma cross-sections

3. Neutron-gamma production cross-sections

4. Neutron yields and spectra

5. Gamma yields and spectra

The neutron cross-sections are produced from the $\mathrm{DLC}-2 \mathrm{D}(4)$ set of 100 group $\mathrm{P}_{8}$ cross-sections based on ENDFB-version III data, obtained from the Radiation Shielding Information Center (RSIC) at the Oak Ridge National Laboratory.

The one dimensional transport code ANISN ${ }^{(5)}$ was used to collapse the cross-sections down to 27 groups. The neutron group structure is shown in Table 1 and is a standard structure used at PNL and elsewhere for many shielding problems. The $\mathrm{P}_{3}$ legendre expansion representation was used for the cross-sections. The ANISN collapse was performed over a combination plutonium fission and $(\alpha, n)$ source spectra moderated through a small plutonium source for plutonium and other source isotopes and through about eight centimeters of hydrogenous shield for shield isotopes. Experience indicates this methodology to be adequate for the types of problems considered and the accuracy necessary. Neutron cross-sections available with the system are shown on Table 2.

The gamma cross-sections were obtained from the Stom-Israel 1ibrary (6) obtained from RSIC and the code MUG ${ }^{(7)}$ was used to produce multigroup $\mathrm{P}_{3}$ cross-sections from the Storm-Israel data. The gamma energy group structure is shown in Table 3. This group structure was obtained from reference $8(8)$ minus the top two groups. The top two groups were not used because the coupled neutron-gamma cross sections use so much computer memory as to 1 imit significantly the number of materials that can be handled per calculation. Also there are practically no gammas produced in these two groups by the sources considered. Gamma cross-sections available with the system are shown on Table 4. 
Neutron-gamma production cross-sections are produced by combining neutron gamma yields and cross-sections. Secondary gamma rays can be produced from many neutron interactions including $(n, \gamma),(n, \alpha),(n, 2 n)$, and inelastic scatter, for example. Only those yields obtainable from the POPOP4 ${ }^{(9)} 1$ ibrary and for which cross-sections were readily obtainable are available in the system. Those secondary gamma cross-sections available with the system and the reactions included are shown in Table 5 . The secondary gamma yield and some inelastic gamma production cross-sections were obtained from the POPOP4 1 ibrary and the $(n, \gamma)$ cross-sections were obtained from GAM-II.

Essentially, the same procedures were followed in producing the $(n, \gamma)$ production cross-sections as are described in the document CTC-20(10) available from RSIC. Much the same approximations and questionable techniques described as such in the document are used. These include the use of thermal neutron induced $(n, \gamma)$ yields for all neutron energies, spectrum weighting of the $(n, \gamma)$ cross-sections not precisely consistent with the weighting used for the neutron cross-sections, and some inelastic gamma production cross-sections used directly from the POPOP4 1ibrary, which may not have been properly spectrum weighted for our purpose.

A small code is provided with the system to combine neutron, gamma and neutron-gamma production cross-sections to obtain a coupled neutron-gamma cross-section. (10) The steps followed and described above are shown in Figure 1 .

A special subroutine has been developed to automatically calculate the neutron and gamma yields and spectra for a given plutonium mass, isotopic composition and age in the Monte Carlo program. The machine solution to the nuclide chain equations is based on the method described by Vondy in ORNL-TM-361. (11) The decay chains (12) are shown in Figure 2.

Yields and spectra for (alpha, neutron) reactions in some common plutonium compounds are also included. These compounds are $\mathrm{PuO}_{2}, \mathrm{PuC}, \mathrm{PuF}_{4}$, $\mathrm{PuBe}$, and $\mathrm{Pu}\left(\mathrm{NO}_{3}\right)$. The sources for this data consist of theoretical 
calculations and experimental measurements performed by PNL and other sources from the open 1iterature. $(13,14,15,16)$ Table 6 1ists the compounds and the yields available in the system. The neutron fission and $(\alpha, n)$ spectra are shown in Table 7. The yields are based on experimental measurements and theoretical calculations performed at PNL and described in BNWL-1259(13) and BNWL-B-109. (14) The same standard U-235 fission spectra is used for all isotopes.

The PUSHLD code is completely separate and has its own set of yields and spectra for gamma sources from plutonium. This code also calculates dose rates as a function of plutonium mass, geometry and age. A more detailed description of this code is found in the document HEDL-TME 73-89.

Gamna yields and spectra built into the BMC code were taken from PUSHLD. The pointwise spectral data was converted to the proper multigroup form. Spectral and yield data for gamma sources built into the code are given in Table 8. 


\section{Calculations and Correlations}

Various calculations have been performed with all parts of the system in order to ensure that the various options are operating correctly and for comparison with experimental results. Most of these calculations were performed for neutron sources, some for gamma sources and less for secondary neutron-gamma sources. Generally, the results when compared with experiments are quite satisfactory for both neutron and gamma dose from plutonium sources. Unfortunately, very little experimental correlation has been performed with the neutron-gamma reaction sources except to ensure that the option works. The data for the neutron-gamma reaction calculations is also the weakest portion of the nuclear data built into the code.

The majority of the calculations performed have concerned neutron dose rate calculations. These have included a simple one dimensional comparison calcuiation with the one dimensional transport code ANISN and with corresponding experimental results, and the modeling of a fairly complex three-dimensional experiment. Also, calculations were performed to compare with experimental measurements performed at Rocky Flats and Burlington for the Division of Operational Safety. Also, calculations have been performed on the model of a glove box line in a concrete building to evaluate the possible affects of neutron scatter off of walls and floor on neutron dose rates.

The first experiment consisted of a $\hat{C} \hat{T}-252$ source centered in spinerical shells of polyethylene of varying thicknesses. A tissue equivalent proportional counter (TEPC) was used to measure the dose rate outside of the shield shells. Calculations of the problem were performed with both ANISN and BMC. Results are shown on Table 9. Both sets of experimental results are well within possible experimental error.

The next experimental setup is shown in Figure 3. This consists of a glove box in a large concrete floured and walled room with removable shields of varying thicknesses of polyethylene surrounding the box. Sources used included a point $\mathrm{Cf}-252$ source and six cans of $\mathrm{PuO}_{2}$ with the isotopic composition shown in Table 10 . The six cans were stacked in two columns 
parallel to the face of the glove box and centered relative to the face as shown in Figure 3. Each can contained about $1 \mathrm{~kg}$ of $\mathrm{PuO}_{2}$ and the total amount consisted of $5.7 \mathrm{~kg}$. A TEPC was used for the dose rate measurements. $\mathrm{A} \mathrm{PuO}_{2}$ source was chosen for its availability and higher source strength which allowed better measurement accuracy to be achieved with shorter counting times.

BMC-MG is capable of modeling the experimental glove box geometry, sources and room geometry in detail. The source strengths and yields are based on data fed into the code concerning the isotopic composition and mass. The limiting considerations are the nuclear data and how well the isotopic composition and masses of the sources are known. Shield composition is also important but is generally well known.

The comparison of calculated and experimental results is shown in Table 11. The calculated results are within nossible experimental errors. The results are in reasonable agreement.

Further calculations were performed in conjunction with experimental measurements of neutron yields and dose rates from relatively simple geometry unshielded plutonium sources at Rocky Flats and Burlington. A Long Counter was used to measure neutron yield and the TEPC for dose rates. The results are shown in Table 12 .

The sources labeled as runs $2,3,4$ and 5 consisted only of Pu metal. The sources for runs $6,7, B-2, B-3$, and B-4 consisted of a combination of fission neutrons and $(\alpha, n)$ neutrons from beryllium. The composition of sources $B$ and $B-1$ were assumed to consist of uranium and plutonium although the composition was not known very well.

The BMC code vias able to model the geometries reasonably accurately except for run 1 and calculate the source yield reasonably accurately for those sources consisting of $\mathrm{Pu}$ metal only. This is shown in Table 1?. The results for runs $B$ and $B-1$ can be expected to contain errors because of probable inaccurate basic information relative to size, mass and composition.

The run 5 source is similar in shape to those of runs $6,7, B-2, B-3$ and $B-4$ however the latter have a beryllium component which produces $(\alpha, n)$ neutrons from the a decay of the plutonium. Since no previous experience 
or data was available to estimate the $(\alpha, n)$ yield for the geometries considered, the total yield could not be calculated. However, by assuming the fission yield could be calculated accurately as shown by the results from runs 2 through 5, it was possible to estimate the $(\alpha, n)$ yields for the several sources.

By calculating the multiplication effect of neutrons born in the plutonium and those born in the beryllium and normalizing to the total measured yield, the $(\alpha, n)$ yields shown in Table 12 were obtained. These should prove useful for assigning $(\alpha, n)$ yields when similar problems need to be calculated without the aid of previous experiments. It is obvious that the $(\alpha, n)$ yields vary greatly. Some of this can be explained by the age of the sources. The sources 6 and 7 were but recently fabricated, B-2 is about two years old and B-3 and B-4 are about ten years old. Assuming a nominal starting isotopic composition as shown in Table 13, the per cent of the $\alpha$ activity accounted for by the buildup of Am-241 increases with time. This is illustrated by Table 14. The BMC-MG code can calculate the build in of Am-241 with time and the effect on $(\alpha, n)$ yields for the plutonium compounds listed in the nuclear data section. For special cases as above the yields would have to be added mechanically.

Other reasons for the greater source strength of B-3 and B-4 can be postulated. One possiblity is that we do not know the isotopic composition accurately. Another possibility is that metallurgical processes have taken place over the years which have effectively increased the surface area where the plutonium and beryllium are in contact and thereby increased the $(\alpha, n)$ source. For instance, this might be caused by the migration of one material into the other.

Calculations were also performed to evaluate the possible effect of scattering off of walls, floors, etc. on dose rates from a glove box 1 ine in a concrete building. The glove box line is shown in Figures 4 and 5 . Polyethylene shielding was placed on the fronts of the boxes only. The results based on calculated dose rates at the detector points shown on Figure 5 are presented in Figure 6 . This shows that in some cases at least, the effect of scattered neutrons is large. 
Some gamma calculations have also been performed with the BMC code and many more with PUSHLD. As an example the experimental set up shown in Figure 7 was modeled with both BMC and PUSHLD. The isotopic composition is shown in Table 15 and the plutonium age from the time the isotopic composition was determined as 40 months. The results for a few shields, shown in Table 16 are not as encouraging as the neutron results. However, the nuclear data such as gamma spectra are now known as well. Also, the experimental results for lead are expected to be high by $10-20 \%$ because of over reaction of the detector to $x$-rays produced in the lead.

PUSHLD has been tested extensively against experimental data. This data is available in the document HEDL-TME 73-89.

Dose rate calculations were performed for use in the analytical study "Combined Plutonium Production and Processing Operations" performed by the AECOP. These calculations were for relatively simple sources shielded by thin shields expected to be encountered in glove box facilities. The sources and shields are described in Tables 17 and 18. Calculated dose rates are shown in Tables 19-22 and the source isotopic composition of the metal in Table 23. The gamma dose rates were calculated with PUSHLD and the neutron dose rates with $B M C-M G$.

Recently a disagreement between our PUSHLD results and Rocky Flats hand calculations has come to our attention. Rocky Flats expects a larger proportion of the dose rate to be due to Am-241 than the PUSHLD calculations predict. The time dependence is the same. The results provided to AECOP have been adjusted, as requested, to reflect this difference in order to ensure conservatism. The results accompanying this report have not been adjusted.

Essentially, there are no comparisons of $(n, \gamma)$ source calculations with experimental results. This portion has been operationally tested however, generally the data used are similar to that used by others and have the same weaknesses. Basically, there is a lack of data needed for $(n, \gamma)$ calculations in many instances. The need to use $(n, \gamma)$ thermal neutron yields for fast neutrons is one example. 


\section{REFERENCES}

1. H. H. Van Tuyl and J. N. Strode, PUSHLD: A Code for Calculation of Gamma Dose Rates from Plutonium in Various Geometries, HEDL-TME 73-89, 1974.

2. D. L. Broder, et a1., "Transmission of Gamma Radiation Through Heterogeneous Media," translated from Atomnaya Energiya, vol. 12, no. 1, pp. 30-35, Jan., 1962.

3. D. H. Thomsen and T. M. Trayer, BMC-1: The Battelle Monte Carlo Code, BNWL-1433, June, 1970. (The multigroup version used in the system was developed from this code.)

4. 100 Group Neutron Cross-section Data Based on ENDF/B, DLC-2D, RSIC Data Library Collection, Oak Ridge, Tenn.

5. W. W. Engle, Jr. A Users Manual For ANISN, K1693, Oak Ridge National Laboratory, AEC contract W7405 eng. 26.

6. Storm-Israel Evaluated Photon Interaction Library, DLC-15, RSIC Data Library Collection, Oak Ridge, Tenn.

7. J. R. Knight and F. R. Mynatt, Mug: A Program For Generating Multigroup Photon Cross-sections, CTC-17, RSIC, Jan, 1970.

8. W. E. Ford, III and D. H. Wallace, POPOP4: A Code For Converting Gamma-Ray Spectra to Secondary Gamma-Ray Production Cross-sections, CTC-12, May, 1969.

9. W. E. Ford, III, The POPOP4 Library of Neutron Induced Secondary Gamma-Ray Yield and Cross-section Data, CTC-42, September, 1970.

10. W. E. Ford, III, and D. H. Wallace, The Use and Testing of AT, Fe, $\mathrm{Ni}, \mathrm{Cu}$, and $\mathrm{Pb}$ Secondary Gamma Ray Production Data Sets From the POPOP4 Library, CTC-20, JuTy 1, 1970.

11. D. R. Vondy, Development of a General Method of Explicit Solution to the Nuclide Chain Equations for Digital Machine Calculations, ORNLTM-361, 1963.

12. H. H. Van Tuyl, Calculation of Gamma Dose Rates at the Surface of Plutonium 0xide Sources, BNWL-1259, January 1970.

13. L. W. Brackenbush and L. G. Faust, Neutron Spectra of Plutonium Compounds, Part 1: He-2 and Li-6 Spectrometer Measurements, BNWL-1262, February, 1970. 
14. D. L. Lessor and R. E. Schenter, Neutron Spectra From $(\alpha, n)$ Reactions in Plutonium Compounds Calculated From Hauser-Feshbach Reaction Theory, BNWL-B-109, June 1971 .

15. T. R. Herold, "Neutron Spectrum of ${ }^{238}$ PuF $_{4}$," Nuclear Instruments and Methods, N, 40-44, 1969.

16. L. Van Der Zwan, "Calculated Neutron Spectra from ${ }^{9} B(\alpha, n)$ Sources," Canadian Journal of Physics, 46, 1527, 1968. 


\section{Table 1}

Neutron Energy Group Structure Used by System

\begin{tabular}{c|c}
\begin{tabular}{c|c} 
Group \\
No.
\end{tabular} & $\begin{array}{c}\text { Upper } \\
\text { Energy (ev) }\end{array}$ \\
\hline 1 & $14.620 \times 10^{6}$ \\
2 & $12.210 \times 10^{6}$ \\
3 & $10.000 \times 10^{6}$ \\
4 & $8.187 \times 10^{6}$ \\
5 & $6.703 \times 10^{6}$ \\
6 & $5.488 \times 10^{6}$ \\
7 & $4.493 \times 10^{6}$ \\
8 & $3.679 \times 10^{6}$ \\
9 & $3.012 \times 10^{6}$ \\
10 & $2.466 \times 10^{6}$ \\
11 & $2.019 \times 10^{6}$ \\
12 & $1.653 \times 10^{6}$ \\
13 & $1.353 \times 10^{6}$ \\
14 & $1.108 \times 10^{6}$ \\
15 & $9.072 \times 10^{5}$ \\
16 & $6.081 \times 10^{5}$ \\
17 & $4.076 \times 10^{5}$ \\
18 & $1.119 \times 10^{5}$ \\
19 & $1.503 \times 10^{4}$ \\
20 & $3.355 \times 10^{3}$ \\
21 & $5.829 \times 10^{2}$ \\
22 & $1.013 \times 10^{2}$ \\
23 & $2.902 \times 10^{1}$ \\
24 & $1.068 \times 10^{1}$ \\
25 & 3.059 \\
26 & 1.125 \\
27 & .4139 \\
&
\end{tabular}


$-19-$

Table 2

27 Group

Neutron Cross-Sections

in System Library

\begin{tabular}{c|l} 
ID No. & Isotope \\
\hline 1 & PU-238 \\
2 & Pu-239 \\
3 & PU-240 \\
4 & Pu-241 \\
5 & Pu-242 \\
6 & Cr \\
7 & Ni \\
8 & Fe \\
9 & Pb \\
10 & Be-9 \\
11 & H \\
12 & 0 \\
13 & C \\
14 & Concrete (ARH-600) \\
15 & B-10 \\
16 & Si \\
17 & Ca \\
18 & Na \\
19 & Al \\
20 & Cl \\
21 & N \\
22 & U-235 \\
23 & U-?.38 \\
24 & Li-6 \\
25 & Li-7 \\
26 & F \\
\end{tabular}




\section{$\underline{T a b l e ~} 3$}

Gamma Energy Group Structure Used By System

\begin{tabular}{c|l} 
No. & $\begin{array}{l}\text { Upper } \\
\text { Energy (ev) }\end{array}$ \\
\hline 1 & \\
2 & $7.0 \times 10^{6}$ \\
3 & $6.0 \times 10^{6}$ \\
4 & $5.0 \times 10^{6}$ \\
5 & $4.0 \times 10^{6}$ \\
6 & $3.5 \times 10^{6}$ \\
7 & $3.0 \times 10^{6}$ \\
8 & $2.5 \times 10^{6}$ \\
9 & $2.0 \times 10^{6}$ \\
10 & $1.6 \times 10^{6}$ \\
11 & $1.2 \times 10^{6}$ \\
12 & $0.9 \times 10^{6}$ \\
13 & $0.6 \times 10^{6}$ \\
14 & $0.4 \times 10^{6}$ \\
15 & $0.21 \times 10^{6}$ \\
16 & $0.12 \times 10^{6}$ \\
17 & $0.07 \times 10^{6}$ \\
& $0.01 \times 10^{6}$
\end{tabular}


$-21-$

Table 4

16 Group Gamma Cross-Sections in System Library

\begin{tabular}{|c|c|}
\hline ID No. & Element \\
\hline 1 & $\mathrm{Pu}$ \\
\hline 2 & $\mathrm{Cr}$ \\
\hline 3 & $\mathrm{Ni}$ \\
\hline 4 & $\mathrm{Fe}$ \\
\hline 5 & $\mathrm{~Pb}$ \\
\hline 6 & $\mathrm{Be}$ \\
\hline 7 & $H$ \\
\hline 8 & 0 \\
\hline 9 & C \\
\hline 10 & B \\
\hline 11 & Si \\
\hline 12 & $\mathrm{Ca}$ \\
\hline 13 & $\mathrm{Na}$ \\
\hline 14 & Al \\
\hline 15 & $\mathrm{Cl}$ \\
\hline 16 & $N$ \\
\hline 17 & U \\
\hline 18 & $\mathrm{Li}$ \\
\hline 19 & $F$ \\
\hline
\end{tabular}


Table 5

Gamma Production Cross-Sections

Available in System Library

\begin{tabular}{|c|c|c|c|}
\hline & Isotope or & Reactions I & luded \\
\hline No. & Element & Inelastic & $(n, Y)$ \\
\hline 1 & $\mathrm{Pu}$ & & $\checkmark$ \\
\hline 2 & $\mathrm{Pu}-240$ & & $\checkmark$ \\
\hline 3 & $\mathrm{Cr}$ & & $\checkmark$ \\
\hline 4 & $\mathrm{Ni}$ & & \\
\hline 5 & $\mathrm{Fe}$ & $\checkmark$ & $\checkmark$ \\
\hline 6 & $\mathrm{~Pb}$ & $\checkmark$ & $\checkmark$ \\
\hline 7 & $\mathrm{Be}-9$ & & $\checkmark$ \\
\hline 8 & $H$ & & $\checkmark$ \\
\hline 9 & 0 & & $\checkmark$ \\
\hline 10 & C & & $\checkmark$ \\
\hline 11 & $B-10$ & & $\checkmark$ \\
\hline 12 & Si & $\checkmark$ & $\checkmark$ \\
\hline 13 & $\mathrm{Ca}$ & $\checkmark$ & $\checkmark$ \\
\hline 14 & $\mathrm{Na}$ & $\checkmark$ & $\checkmark$ \\
\hline 15 & A1 & & $\checkmark$ \\
\hline 16 & Cl & $\checkmark$ & $\checkmark$ \\
\hline 17 & N & $\checkmark$ & $\checkmark$ \\
\hline 18 & $U-235$ & & $\checkmark$ \\
\hline 19 & $U-238$ & & $\checkmark$ \\
\hline 20 & Li-6 & & $\checkmark$ \\
\hline 21 & $\mathrm{~F}$ & & $\checkmark$ \\
\hline
\end{tabular}




\section{Table 6}

Neutron Yields

$\mathrm{n} / \mathrm{sec}$ gram of $\mathrm{Pu}$

$(a, n)$ yields

\begin{tabular}{|c|c|c|c|c|c|c|}
\hline Isotope & $\mathrm{PuO}_{2}$ & $\mathrm{PuF}_{4}$ & PuC & PuBe $(n)$ & $\mathrm{Pu}\left(\mathrm{NO}_{3}\right)^{(\mathrm{b})}$ & $\begin{array}{c}\text { Spontaneous } \\
\text { Fission } \\
\end{array}$ \\
\hline Pu 236 & - & - & - & - & - & $3.7 \times 10^{4}$ \\
\hline Pu 238 & $1.34 \times 10^{4}$ & $2.04 \times 10^{6}$ & $1.49 \times 10^{4}$ & $4.38 \times 10^{7}$ & $2.7 \times 10^{4}$ & $2.62 \times 10^{3}$ \\
\hline Pu 239 & 38 & $6.35 \times 10^{3}$ & 37.3 & $1.36 \times 10^{5}$ & 76 & .03 \\
\hline Pu 240 & $1.45 \times 10^{2}$ & $2.35 \times 10^{4}$ & 137.8 & $5.02 \times 10^{5}$ & $2.9 \times 10^{2}$ & $1.02 \times 10^{3}$ \\
\hline Pu 241 & - & - & - & - & - & - \\
\hline Pu 242 & 2.13 & $2.83 \times 10^{2}$ & - & - & 4.2 & $1.7 \times 10^{3}$ \\
\hline Am 241 & $2.68 \times 10^{3}$ & $4.08 \times 10^{5}$ & $2.98 \times 10^{3}$ & $8.76 \times 10^{6}$ & $5.46 \times 10^{3}$ & - \\
\hline
\end{tabular}

(a) Estimated from Reference 16.

(b) A rough estimate indicates an approximate doubling of $(\alpha, n)$ yields compared to $\mathrm{PuO}_{2}$. 
$\underline{\text { Table } 7}$

Neutron Source Spectra Built Into System

\begin{tabular}{|c|c|c|c|c|c|c|}
\hline \multirow[b]{2}{*}{ Group No. } & \multirow{2}{*}{$\begin{array}{l}\text { Spontaneous } \\
\text { Fission }\end{array}$} & \multicolumn{4}{|c|}{$(\alpha, n)$ Spectra } & \multirow[b]{2}{*}{$\mathrm{Pu}\left(\mathrm{NO}_{3}\right)_{\mathrm{n}}$} \\
\hline & & $\mathrm{PuO}_{2}$ & $\mathrm{PuF}_{4}$ & PuC & PuBe & \\
\hline 1 & $1.502 \times 10^{-4}$ & 0.0 & 0.0 & 0.0 & 0.0 & 0.0 \\
\hline 2 & $8.866 \times 10^{-4}$ & 0.0 & 0.0 & 0.0 & .009 & 0.0 \\
\hline 3 & $3.455 \times 10^{-3}$ & 0.0 & 0.0 & 0.0 & 0.0746 & 0.0 \\
\hline 4 & $9.806 \times 10^{-3}$ & 0.0 & 0.0 & 0.0451 & 0.137 & 0.0 \\
\hline 5 & $2.151 \times 10^{-2}$ & 0.0 & 0.0 & 0.169 & 0.105 & 0.0 \\
\hline 6 & $3.830 \times 10^{-2}$ & 0.0 & 0.0 & 0.322 & 0.162 & 0.0 \\
\hline 7 & $5.748 \times 10^{-2}$ & 0.026 & 0.0 & 0.317 & 0.133 & 0.026 \\
\hline 8 & $7.534 \times 10^{-2}$ & 0.133 & 0.0 & 0.146 & 0.145 & 0.133 \\
\hline 9 & $2.834 \times 10^{-2}$ & 0.254 & 0.015 & 0.0006 & 0.097 & 0.254 \\
\hline 10 & $9.477 \times 10^{-2}$ & 0.232 & 0.100 & 0.0 & 0.050 & 0.232 \\
\hline 11 & $9.466 \times 10^{-2}$ & 0.159 & 0.198 & 0.0 & 0.027 & 0.159 \\
\hline 12 & $8.935 \times 10^{-2}$ & 0.089 & 0.246 & 0.0 & 0.012 & 0.089 \\
\hline 13 & $8.023 \times 10^{-2}$ & 0.049 & 0.114 & 0.0 & 0.012 & 0.049 \\
\hline 14 & $6.965 \times 10^{-2}$ & 0.026 & 0.094 & 0.0 & 0.009 & 0.026 \\
\hline 15 & $1: 068 \times 10^{-1}$ & 0.020 & 0.123 & 0.0 & 0.020 & 0.020 \\
\hline 16 & $6.962 \times 10^{-2}$ & 0.006 & 0.061 & 0.0 & 0.006 & 0.006 \\
\hline 17 & $8.513 \times 10^{-2}$ & 0.005 & 0.046 & 0.0 & 0.0 & 0.005 \\
\hline 18 & $1.555 \times 10^{-2}$ & 0.0 & 0.003 & 0.0 & 0.0 & 0.0 \\
\hline 19 & $7.494 \times 10^{-4}$ & 0.0 & 0.0 & 0.0 & 0.0 & 0.0 \\
\hline 20 & $8.237 \times 10^{-5}$ & 0.0 & 0.0 & 0.0 & 0.0 & 0.0 \\
\hline 21 & $5.972 \times 10^{-6}$ & 0.0 & 0.0 & 0.0 & 0.0 & 0.0 \\
\hline 22 & $3.950 \times 10^{-7}$ & 0.0 & 0.0 & 0.0 & 0.0 & 0.0 \\
\hline 23 & $5.556 \times 10^{-8}$ & 0.0 & 0.0 & 0.0 & 0.0 & 0.0 \\
\hline 24 & $1.352 \times 10^{-8}$ & 0.0 & 0.0 & 0.0 & 0.0 & 0.0 \\
\hline 25 & $1.902 \times 10^{-9}$ & 0.0 & 0.0 & 0.0 & 0.0 & 0.0 \\
\hline 26 & $4.242 \times 10^{-10}$ & 0.0 & 0.0 & 0.0 & 0.0 & 0.0 \\
\hline 27 & $1.218 \times 10^{-10}$ & 0.0 & 0.0 & 0.0 & 00 & 0.0 \\
\hline
\end{tabular}


Table 8

Gamma Spectra and Yields by Group

gammas/100 disintegrations

\begin{tabular}{|c|c|c|c|c|c|c|c|c|c|c|c|c|c|c|}
\hline $\begin{array}{l}\text { Group } \\
\text { No. } \\
\end{array}$ & $P u-238$ & $\underline{P u-239}$ & $P u-240$ & $P_{u-241}$ & $\underline{P u-242}$ & Am-241 & $\underline{U}-237$ & $\underline{P u-236}$ & $\underline{U}-232$ & Th-228 & $\underline{R a-224}$ & $\underline{\mathrm{Pb}-212^{(\mathrm{a})}}$ & $\underline{B i-212^{(b)}}$ & $\begin{array}{l}\text { Fission } \\
\text { Spectra }(c)\end{array}$ \\
\hline 1 & 15.25 & 6.65 & 13.24 & $3.6-4$ & 13.27 & 93.14 & 80.71 & $3.1-3$ & 30.2 & 25.0 & 0.0 & 0.0 & 1.5 & $1.4-3$ \\
\hline 2 & $8.2-3^{(d)}$ & $6.8-3$ & $1.2-2$ & $1.7-3$ & 0.0 & 0.047 & 56.6 & $1.2-2$ & 0.0 & 1.6 & 0.0 & 33.7 & 0.0 & $2.8-3$ \\
\hline 3 & $1.0-3$ & $4.9-3$ & $1.2-3$ & $1.6-4$ & 0.0 & $5.9-3$ & 25.0 & $6.6-5$ & $8.2-2$ & 0.30 & 0.0 & 0.24 & 0.0 & $8.3-3$ \\
\hline 4 & 0.0 & $2.88-3$ & 0.0 & 0.0 & 0.0 & $1.7-3$ & 2.4 & 0.0 & $7.2-3$ & 0.29 & 4.2 & 50.6 & 4.6 & $9.1-3$ \\
\hline 5 & 0.0 & $1.23-3$ & 0.0 & 0.0 & 0.0 & $9.0-5$ & 0.0 & $2.8-4$ & 0.0 & 0.0 & 0.0 & 0.16 & 40.3 & $1.6-2 N$ \\
\hline 6 & $5.5-5$ & $9.4-5$ & $1.8-5$ & 0.0 & 0.0 & $1.0-3$ & 0.0 & $2.4-4$ & 0.0 & 0.0 & 0.0 & 0.0 & 13.5 & $2.7-2$ \\
\hline 7 & $8.0-7$ & 0.0 & 0.0 & 0.0 & 0.0 & 0.0 & 0.0 & 0.0 & 0.0 & 0.0 & 0.0 & 0.0 & 1.0 & $4.7-2$ \\
\hline 8 & 0.0 & 0.0 & 0.0 & 0.0 & 0.0 & 0.0 & 0.0 & 0.0 & 0.0 & 0.0 & 0.0 & 0.0 & 0.29 & $6.1-2$ \\
\hline 9 & 0.0 & 0.0 & 0.0 & 0.0 & 0.0 & 0.0 & 0.0 & 0.0 & 0.0 & 0.0 & 0.0 & 0.0 & 1.7 & $9.9-2$ \\
\hline 10 & 0.0 & 0.0 & 0.0 & 0.0 & 0.0 & 0.0 & 0.0 & 0.0 & 0.0 & 0.0 & 0.0 & 0.0 & 0.0 & $1.2-1$ \\
\hline 11 & 0.0 & 0.0 & 0.0 & 0.0 & 0.0 & 0.0 & 0.0 & 0.0 & 0.0. & 0.0 & 0.0 & 0.0 & 36.0 & $1.9-1$ \\
\hline 12 & 0.0 & 0.0 & 0.0 & 0.0 & 0.0 & 0.0 & 0.0 & 0.0 & 0.0 & 0.0 & 0.0 & 0.0 & 0.0 & $1.6-1$ \\
\hline 13 & 0.0 & 0.0 & 0.0 & 0.0 & 0.0 & 0.0 & 0.0 & 0.0 & 0.0 & 0.0 & 0.0 & 0.0 & 0.0 & $1.7-1$ \\
\hline 14 & 0.0 & 0.0 & 0.0 & 0.0 & 0.0 & 0.0 & 0.0 & 0.0 & 0.0 & 0.0 & 0.0 & 0.0 & 0.0 & $8.6-2$ \\
\hline 15 & 0.0 & 0.0 & 0.0 & 0.0 & 0.0 & 0.0 & 0.0 & 0.0 & 0.0 & 0.0 & 0.0 & 0.0 & 0.0 & $5.0-2$ \\
\hline 16 & 0.0 & 0.0 & 0.0 & 0.0 & 0.0 & 0.0 & 0.0 & 0.0 & 0.0 & 0.0 & 0.0 & 0.0 & 0.0 & $5.1-2$ \\
\hline & & 0.0 & & & & & & & & & & & & \\
\hline 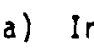 & des gamma & $s$ from $P$ & 16 and & $1-220$ & & & & & & & & & & \\
\hline b) In & Jdes gamma & $s$ from $P$ & 12 and & 208 & & & & & & & & & & \\
\hline $\begin{array}{l}\text { c) } \frac{S}{W} \\
\text { d) } R\end{array}$ & $\begin{array}{l}\text { tra normal } \\
\text { includes } \\
\text { as } 8.2 \times\end{array}$ & $\begin{array}{l}\text { zed to } \\
\text { prompt } \\
0^{-3}\end{array}$ & $\begin{array}{r}\text { yfelc } \\
\text { ftssic }\end{array}$ & $\begin{array}{l}n \text { BMC- } \\
\text { roduct }\end{array}$ & $\begin{array}{l}\text { qual t } \\
\text { mas. }\end{array}$ & & ssion & & & & & & & \\
\hline
\end{tabular}




\section{Table 9}

Ratio of Calculated Kerma to Experimental Kerma

\begin{tabular}{l|c|c}
${ }^{252}$ Cf Source with & \\
Spherical Shield & ANISN & BMC \\
\hline 2" Poly Sphere & 0.998 & $1.036 \pm 0.015$ \\
3" Poly Sphere & 1.000 & $1.016 \pm 0.012$ \\
4" Poly Sphere & 1.080 & $1.125 \pm 0.013$
\end{tabular}

\section{Table 10}

Isotopic Plutonium Composition (a) For Source in Neutron Dose Rate Measurement Experiment

\begin{tabular}{l|c} 
Isotope & wt\% \\
\hline Pu 236 & negligible \\
Pu 238 & 0.092 \\
Pu 239 & 70.567 \\
Pu 240 & 23.909 \\
Pu 241 & 4.174 \\
Pu 242 & 1.258 \\
Am 241 & 20.098 \\
U 237 & -
\end{tabular}

(a) Plutonium from reprocessed LWR fuel 
Table 11

Comparison of Calculations To Experimental

Measurements of Neutron Kerma Rate From a Glove Box

\begin{tabular}{|c|c|c|c|c|}
\hline & \multicolumn{2}{|c|}{$C f-252$} & \multicolumn{2}{|c|}{$\mathrm{PuO}_{2}$} \\
\hline $\begin{array}{c}\text { Thickness } \\
\text { of Polyethylene }\end{array}$ & $\begin{array}{r}\text { Experi } \\
\downarrow\end{array}$ & Calculation & Experim & Calculation \\
\hline 2 inches & 0.473 & $0.528 \pm 0.002$ & 0.464 & $0.466 \pm 0.0015$ \\
\hline 4 inches & 0.228 & $0.227 \pm 0.014$ & 0.192 & $0.212 \pm 0.022$ \\
\hline $\begin{array}{l}4 \text { inches on front } \\
2 \text { inches on back, } \\
\text { sides and bottom }\end{array}$ & 0.210 & $0.207 \pm 0.03$ & & \\
\hline
\end{tabular}




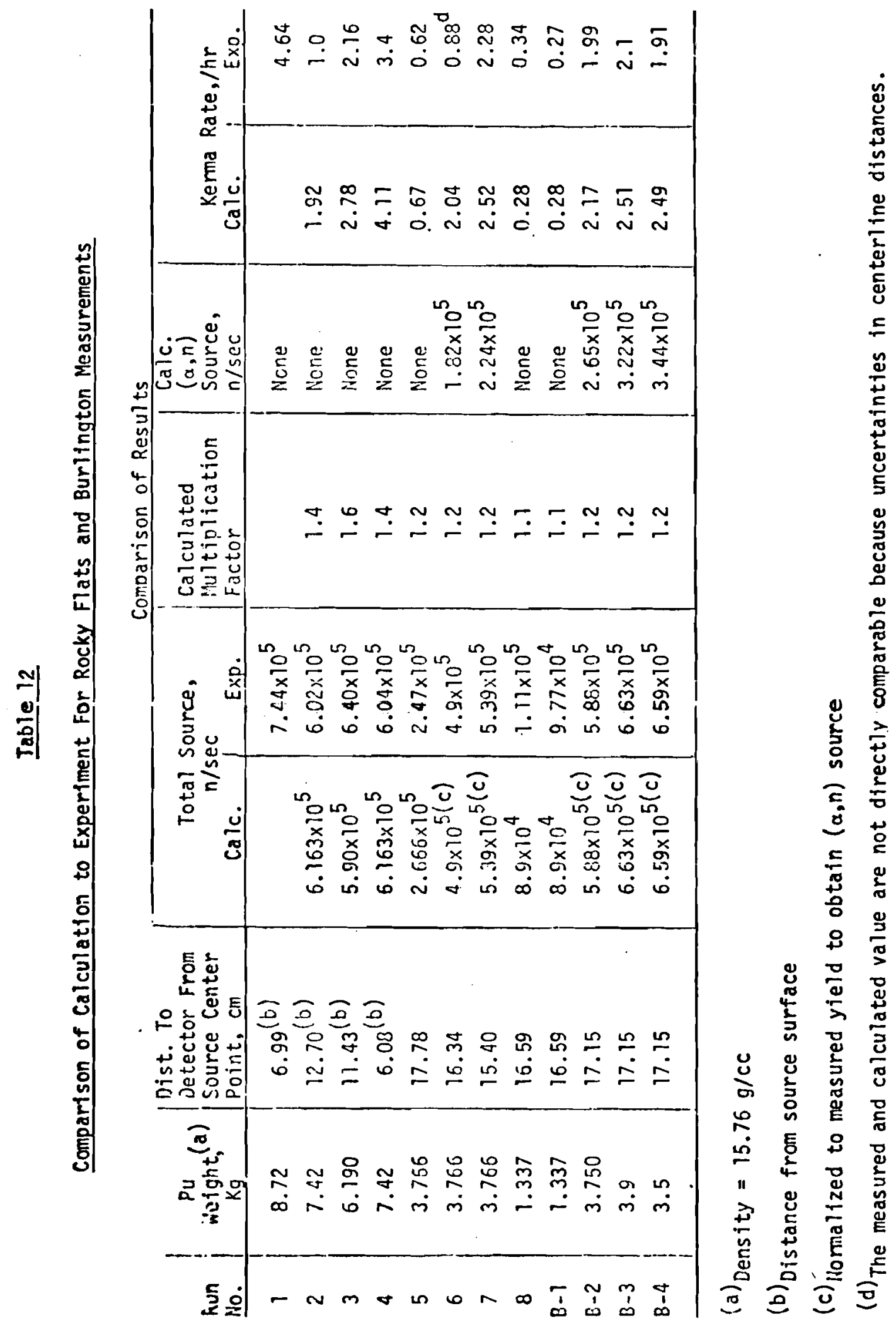


Table 13

Plutonium Isotopic Composition For Rocky Flats and Burlington Measurements

\begin{tabular}{c|c} 
Isotope & wt $\%$ \\
\hline Pu-239 & 93.48 \\
Pu-240 & 6.0 \\
Pu-241 & 0.5 \\
Am-241 & 0.02 \\
TOTAL & 100.
\end{tabular}

Table 14

a Activity From Am-241 as a Function of Plutonium Age For Plutonium Composition of Table 13

\begin{tabular}{c|c|c}
$\begin{array}{c}\text { Plutonium } \\
\text { Age, } \\
\text { years }\end{array}$ & $\begin{array}{c}\text { Am-241 Cortent, } \\
\text { wt\% }\end{array}$ & $\begin{array}{c}\text { Percent of Total } \\
\text { Activity in the Fu } \\
\text { Due to Am-241 }\end{array}$ \\
\hline 0 (a) & 0.02 & 0.9 \\
1 & 0.04 & 1.8 \\
2 & 0.06 & 2.7 \\
5 & 0.11 & 4.8 \\
10 & 0.17 & 7.3 \\
20 & 0.32 & 12.8
\end{tabular}
(a) The isotopic composition at time zero is assumed to be as
shown in Table 13. 


\section{Table 15}

Inftial Pu Isotopic Composition For

Gamma Dose Rate Measurements (a)

\begin{tabular}{l|c} 
Isotope & wt\% \\
\hline Pu-236 & $0.66 \times 10^{-6}$ \\
Pu-238 & 0.37 \\
Pu-239 & 71.3 \\
Pu-240 & 20.8 \\
Pu-241 & 5.97 \\
Pu-242 & 1.58 \\
Am-241 & 0.47 \\
$U-237$ & 0.0
\end{tabular}

(a) Measurements were made 40 months after this composition was experimentally determined. 
Table 16

Comparison of Experimental Measurements and Calculations of PuO 2 Gamma Dose Rates

\begin{tabular}{ccccc} 
Shield & Thickness $(\mathrm{cm})$ & Exp. & PUSHLD & BMC -MG \\
\hline $\mathrm{Pb}$ & 1.35 & 8.4 & 7.1 & $6.0 \pm .4$ \\
$\mathrm{~Pb}$ & 2.52 & 2.8 & 2.0 & $1.9 \pm .1$ \\
$\begin{array}{c}\text { Stainless } \\
\text { Steel }\end{array}$ & 1.35 & 67 & 79 & $56 \pm 4$ \\
$\begin{array}{c}\text { Stainless } \\
\text { Steel }\end{array}$ & 2.57 & 30 & 33 & $29 \pm 4$
\end{tabular}


Table 17

Source Descriptions for AECOP

\begin{tabular}{l|c|c|c} 
Source & Shape & $\begin{array}{c}\text { Dimensions }(\mathrm{cm}) \\
\text { (Dia } \times \text { Thickness) }\end{array}$ & WT $(\mathrm{Kg})$ \\
\hline 1 & Disc & $30.061 \times .760$ & 8.5 \\
2 & Disc & $26.467 \times .346$ & 3.0 \\
3 & Disc & $26.676 \times .193$ & 1.7
\end{tabular}


$\underline{\text { Table } 18}$

Shielding Cases For AECOP

\begin{tabular}{l|c|c|c} 
Case & Material & Thickness $($ in) & Dose Point \\
\hline 1 & Steel & .1225 & 1 foot from source \\
2 & Steel & .1225 & 1 foot from source \\
& Lead & .1250 & \\
3 & Lead Glove & .030 & Shield Surface \\
& & $.1 \mathrm{~mm}$ Pb Equiv. &
\end{tabular}


Table 19

Gamma Dose Rate Calculational Results For AECOP

Dose Rate at 1 Foot

(mrem/hr)

Stainless Steel and Lead (Case No. 2, Table 18)

\begin{tabular}{|c|c|c|c|c|c|c|c|c|c|}
\hline \multirow[b]{2}{*}{ Composition } & \multicolumn{3}{|c|}{ Source $I$ (a) } & \multicolumn{3}{|c|}{ Source 2} & \multicolumn{3}{|c|}{ Source 3} \\
\hline & 0 & $5 \mathrm{Yr}$. & $10 \mathrm{Yr}$ & 0 & $5 \mathrm{Yr}$ & $10 \mathrm{rr}_{r}$ & 0 & $5 Y_{r}$ & $10 \mathrm{Vr}$ \\
\hline Base $P$ & 6.86 & 7.30 & 7.67 & 4.54 & 4.80 & 4.99 & 3.45 & 3.61 & 3.74 \\
\hline$P-1$ & 6.85 & 7.27 & 7.62 & 4.53 & 4.78 & 4.96 & 3.44 & 3.60 & 3.72 \\
\hline P-2 & 6.83 & 7.24 & 7.58 & 4.52 & 4.76 & 4.94 & 3.43 & 3.58 & 3.70 \\
\hline$P-3$ & 6.82 & 7.21 & 7.53 & $4.51^{\circ}$ & 4.73 & 4.91 & 3.42 & 3.57 & 3.67 \\
\hline$P-4$ & 6.80 & 7.17 & 7.49 & 4.50 & 4.71 & 4.88 & 3.41 & 3.55 & 3.65 \\
\hline$P-5$ & 6.79 & 7.14 & 7.44 & 4.49 & 4.69 & 4.85 & 3.40 & 3.53 & 3.63 \\
\hline$P-6$ & 6.76 & 7.08 & 7.35 & 4.46 & 4.65 & 4.79 & 3.38 & 3.50 & 3.59 \\
\hline RA-1 & 6.86 & 7.30 & 7.67 & 4.54 & 4.80 & 4.99 & 3.45 & 3.61 & 3.74 \\
\hline RA-2 & 6.86 & 7.30 & 7.67 & 4.54 & 4.80 & 4.99 & 3.45 & 3.61 & 3.74 \\
\hline RA-3 & 6.86 & 7.30 & 7.66 & 4.54 & 4.79 & 4.99 & 3.45 & 2.61 & 3.73 \\
\hline RA-4 & 6.85 & 7.29 & 7.66 & 4.54 & 4.79 & 4.99 & 3.45 & 3.61 & 3.73 \\
\hline RA-5 & 6.85 & 7.29 & 7.66 & 4.53 & 4.79 & 4.99 & 3.44 & 3.61 & 3.73 \\
\hline$R A-6$ & 6.85 & 7.29 & 7.66 & 4.53 & 4.79 & 4.98 & & & \\
\hline MA-I & 6.86 & 7.30 & 7.67 & 4.54 & 4.80 & 4.99 & 3.45 & 3.61 & 3.74 \\
\hline MA-2 & 6.86 & 7.30 & 7.67 & 4.54 & 4.80 & 4.99 & 3.45 & 3.61 & 3.74 \\
\hline$M A-3$ & 6.86 & 7.30 & 7.66 & 4.54 & 4.80 & 4.99 & 3.45 & 3.61 & 3.73 \\
\hline$M A-4$ & 6.86 & 7.30 & 7.66 & 4.54 & 4.79 & 4.99 & 3.45 & 3.61 & 3.73 \\
\hline MA-5 & 6.86 & 7.29 & 7.66 & 4.54 & 4.79 & 4.99 & 3.45 & 3.61 & 3.73 \\
\hline$M A-6$ & 6.86 & 7.29 & 7.66 & 4.54 & 4.79 & 4.99 & 3.45 & 3.61 & 3.73 \\
\hline RA-HI & 6.88 & 7.32 & 7.69 & 4.55 & 4.81 & 5.00 & 3.46 & 3.62 & 3.74 \\
\hline RMA- 1 & 6.85 & 7.29 & 7.68 & 4.53 & 4.79 & 4.99 & 3.44 & 3.61 & 3.73 \\
\hline RMA-2. & 6.84 & 7.28 & 6.85 & 4.53 & 4.79 & 4.98 & 3.44 & 3.60 & 3.73 \\
\hline
\end{tabular}

(a) Source description - Table 17 
Table 20

Gamma Dose Rate Calculational Results For AECOP

Dose Rate At 1 Foot

(mrem/hr)

Stainless Stee1 (Case No. 1, Table 18)

\begin{tabular}{|c|c|c|c|c|c|c|c|c|c|}
\hline \multirow[b]{2}{*}{ Conoosition } & \multicolumn{3}{|c|}{ Source 7 (a) } & \multicolumn{3}{|c|}{ Source 2} & \multicolumn{3}{|c|}{ Source 3} \\
\hline & 0 & $5 \mathrm{Yr}$. & $10 \mathrm{rr}$ & 0 & $\underline{5 \mathrm{Yr}}$ & $10 \mathrm{Yr}$ & 0 & $5 \mathrm{Yr}$ & $10 \mathrm{Yr}$ \\
\hline Base $P$ & 15.7 & 17.8. & 19.6 & 11.1 & 12.9 & 14.3 & 9.29 & 11.1 & 12.4 \\
\hline$P-1$ & 15.5 & 17.5 & 19.3 & 10.9 & 12.7 & 14.1 & 9.17 & 10.9 & 12.2 \\
\hline$P-2$ & 15.4 & 17.3 & 18.9 & 10.8 & 12.5 & 13.8 & 9.05 & 10.7 & 11.9 \\
\hline P-3 & 15.2 & 17.0 & 18.6 & 10.7 & 12.3 & 13.6 & 8.92 & 10.5 & 11.7 \\
\hline P-4 & 15.1 & 16.8 & 18,3 & 10.6 & 12.1 & 13.3 & 8.80 & 10.3 & 11.4 \\
\hline$P-5$ & 14.9 & 16.6 & 18.0 & 10.4 & 11.9 & 13.1 & 8.68 & 10.1 & 11.2 \\
\hline$P-6$ & 14.6 & 16.1 & 17.4 & 10.2 & 11.5 & 12.6 & 8.44 & 9.73 & 10.7 \\
\hline$P A-1$ & 15.7 & 17.7 & 19.6 & 11.1 & 12.9 & 14.3 & 9.28 & 11.1 & 12.4 \\
\hline$R A-2$ & 15.7 & 17.7 & 19.5 & 11.1 & 12.9 & 14.3 & 9.27 & 11.1 & 12.4 \\
\hline$R A-3$ & 15.7 & 17.7 & 19.5 & 11.0 & 12.9 & 14.3 & 9.26 & 11.0 & 12.4 \\
\hline$R A-4$ & 15.6 & 17.7 & 19.5 & 11.0 & 12.9 & 14.3 & 9.25 & 11.0 & 12.4 \\
\hline$R A-5$ & 15.6 & 17.7 & 19.5 & 11.0 & 12.9 & 14.3 & 9.24 & 11.0 & 12.4 \\
\hline RA-6 & 15.6 & 17.7 & 19.5 & 11.0 & 12.9 & 14.3 & 9.22 & 11.0 & 12.3 \\
\hline$M A-1$ & 15.7 & 17.7 & 19.6 & 11.1 & 12.9 & 14.3 & 9.28 & 11.7 & 72.4 \\
\hline$\because A-2$ & 15.7 & 17.7 & 19.6 & 11.1 & 12.9 & 14.3 & 9.28 & 11.1 & 12.4 \\
\hline$\because A-3$ & 15.7 & 17.7 & 19.5 & 11.0 & 12.9 & 14.3 & 9.27 & 11.1 & 12.4 \\
\hline$\because \dot{\lambda}-4$ & 15.7 & 17.7 & 19.5 & 11.0 & 12.9 & 14.3 & 9.26 & 11.0 & .12 .4 \\
\hline$M A-5$ & 15.7 & 17.7 & 19.5 & 11.0 & 12.9 & 14.3 & 9.26 & 11.0 & 12.4 \\
\hline : & 15.6 & 17.7 & 19.5 & 11.0 & 12.9 & 14.3 & 9.25 & 11.0 & 12.4 \\
\hline RA-HI & 15.8 & 17.9 & 19.7 & 11.2 & 13.0 & 14.4 & 9.37 & 11.2 & 12.5 \\
\hline R'AA-1 & $15.6^{\circ}$ & 17.7 & 19.5 & 11.0 & 12.9 & 14.3 & 9.24 & 11.0 & 12.4 \\
\hline BNA-2 & 15.6 & 17.7 & 19.5 & 11.0 & $12.9^{\circ}$ & 14.3 & 9.24 & 11.0 & $12.4^{\circ}$ \\
\hline
\end{tabular}

(a)Source description - Table 17 
Table 21

Gamma Dose Rate Calculational Results For AECOP

Dose Rates

(Rad/hr)

Lead Glove (No. 3 Shield, Table 18)

\begin{tabular}{|c|c|c|c|c|c|c|c|c|c|}
\hline \multirow[b]{2}{*}{ Comsosition } & \multicolumn{3}{|c|}{ Source 1 (a) } & \multicolumn{3}{|c|}{ Source 2} & \multicolumn{3}{|c|}{ Source 3} \\
\hline & 0 & $5 \gamma r$. & $10 \mathrm{Yr}$ & 0 & $5 \mathrm{Yr}$ & $10 \mathrm{Yr}$ & 0 & $5 \mathrm{Yr}$ & $10 \mathrm{Yr}$ \\
\hline Base P & .178 & .352 & .483 & .169 & .341 & .471 & .154 & .325 & .454 \\
\hline$P-1$ & .176 & .343 & .470 & .167 & .332 & .458 & .153 & .316 & .441 \\
\hline$P-2$ & .175 & .335 & .456 & .165 & .324 & .444 & .151 & .308 & .427 \\
\hline$P-3$ & .173 & .327 & .443 & .164 & .316 & .431 & .149 & .300 & .414 \\
\hline$P-4$ & .171 & .318 & .430 & .162 & .307 & .418 & .148 & .292 & .401 \\
\hline$P-5$ & .170 & .310 & .416 & .160 & .299 & .404 & .146 & .284 & .388 \\
\hline$P-6$ & .166 & .293 & .389 & .157 & .283 & .378 & .143 & .267 & $: 362$ \\
\hline$R A-1$ & .178 & .351 & .483 & .168 & .340 & .470 & .154 & .324 & .453 \\
\hline$R A-2$ & .177 & .351 & .482 & .168 & .340 & .470 & .153 & .324 & .453 \\
\hline$R A-3$ & .176 & .350 & .481 & .167 & .339 & .469 & .153 & .323 & .457 \\
\hline RÁ-G & .175 & .349 & .480 & .166 & .338 & .468 & .152 & .322 & .451 \\
\hline RA-5 & .174 & .348 & .480 & .165 & .337 & .467 & .151 & .321 & .450 \\
\hline RA -6 & .174 & .347 & .479 & .164 & .336 & .466 & .150 & .320 & .449 \\
\hline 1 & .178 & .351 & .483 & .169 & .340 & .471 & .154 & .324 & .453 \\
\hline$B A-2$ & .178 & .351 & .483 & .168 & .340 & .470 & .154 & .324 & .453 \\
\hline$\because A-3$ & .177 & .351 & .482 & .168 & .340 & .470 & .153 & .324 & .453 \\
\hline$\because \dot{3}$ & .176 & .350 & .482 & .167 & .339 & .469 & .153 & .323 & .452 \\
\hline$\because A-5$ & .176 & .350 & .481 & .167 & .339 & .469 & .152 & .323 & .452 \\
\hline Yiti-5 & .175 & .349 & .480 & .166 & .338 & .468 & .152 & .322 & .451 \\
\hline PA-LII & .184 & .358 & .489 & .175 & .347 & .477 & .161 & .331 & .460 \\
\hline RU:LA- & .174 & .348 & .480 & .165 & .337 & .467 & .151 & .321 & .450 \\
\hline Pixid-2 & 171. & .345 & .476 & .162 & .334 & .464 & .147 & .318 & .447 \\
\hline
\end{tabular}

(a) Source description - Table 17 
Table 22

Calculated Neutron Dose Rates For AECOP

(mrem/hr)

\begin{tabular}{|c|c|c|c|c|}
\hline Source & $\begin{array}{l}\text { S.F. } \\
\text { n/sec }\end{array}$ & $\begin{array}{l}\text { Calc. } \\
\text { Mult. } \\
\text { Factor }\end{array}$ & $\begin{array}{l}\text { Total } \\
\text { Source } \\
\text { n/sec }\end{array}$ & $\begin{array}{l}\text { Dose Rates } \\
\text { Surface } \\
\text { Pb Glv (a) }\end{array}$ \\
\hline 1 & $5.20 \times 10^{5}$ & 1.34 & $6.97 \times 10^{5}$ & $105 \pm 17$ \\
\hline 2 & $1.84 \times 10^{5}$ & 1.17 & $2.15 \times 10^{5}$ & $39 \pm 3$ \\
\hline 3 & $1.04 \times 10^{5}$ & $\sim 1.10$ & $1.14 \times 10^{5}$ & $\begin{array}{c}27 \pm 3 \\
\text { Dose Rates at }(\mathrm{a}) \\
1 \mathrm{ft} ; \mathrm{SS} \text { and } \mathrm{PB}\end{array}$ \\
\hline 1 & $5.20 \times 10^{5}$ & 1.69 & $8.79 \times 10^{5}$ & $5.67 \pm .06$ \\
\hline 2 & $1.84 \times 10^{5}$ & 1.24 & $2.29 \times 10^{5}$ & $1.72 \pm .01$ \\
\hline 3 & $1.04 \times 10^{5}$ & 1.13 & $1.18 \times 10^{5}$ & $\begin{array}{l}.90 \pm .01 \\
1 \text { foot } \\
\text { No shields }\end{array}$ \\
\hline 1 & $5.20 \times 10^{5}$ & 1.35 & $7.0 \times 10^{5}$ & $4.92 \pm .06$ \\
\hline 2 & $1.84 \times 10^{5}$ & 1.16 & $2.13 \times 10^{5}$ & $1.46 \pm .01$ \\
\hline 3 & $1.04 \times 10^{5}$ & - & - & 1.2 (est.) \\
\hline
\end{tabular}

(a) For source and shield descriptions see Tables 17 and 18. 
Table 23

Plutonium Metal Isotopic Compositions (a) Used for AECOP Calculations

\begin{tabular}{l|l|l} 
Case & \multicolumn{2}{|c|}{ Weight } \\
& Pu 241 & Am 241 \\
\hline & & \\
Base P & 0.5080 & 0.0190 \\
P-1 & 0.4886 & 0.0187 \\
P-2 & 0.4692 & 0.0184 \\
P-3 & 0.4498 & 0.0182 \\
P-4 & 0.4304 & 0.0179 \\
P-5 & 0.4109 & 0.0177 \\
P-6 & 0.3721 & 0.0172 \\
RA-1 & 0.5080 & 0.0187 \\
RA-2 & 0.5080 & 0.0184 \\
RA-3 & 0.5080 & 0.0178 \\
RA-4 & 0.5080 & 0.0172 \\
RA-5 & 0.5080 & 0.0166 \\
RA-6 & 0.5080 & 0.0160 \\
MA-1 & 0.5080 & 0.0188 \\
MA-2 & 0.5080 & 0.0186 \\
MA-3 & 0.5080 & 0.0183 \\
MA-4 & 0.5080 & 0.0179 \\
MA-5 & 0.5080 & 0.0176 \\
MA-6 & 0.5080 & 0.0172 \\
RA-HI & 0.5080 & 0.0231 \\
RMA-1 & 0.5080 & 0.0166 \\
RMA-2 & 0.5080 & 0.0143 \\
& &
\end{tabular}

(a) Rest of the composition is 6 wt\% Pu-241 plus Pu-239 needed to make $100 \mathrm{wt} \%$ 


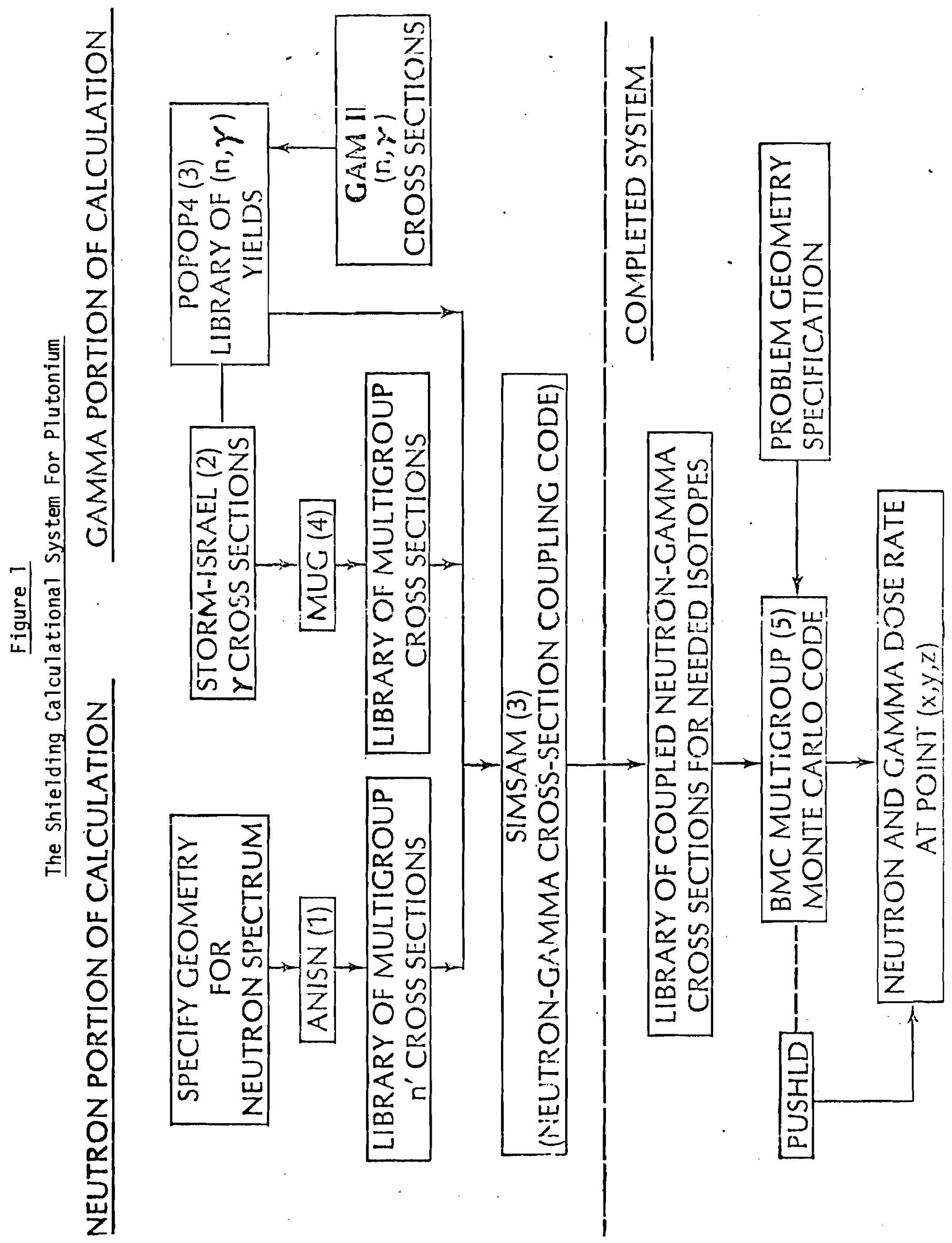


Figure 2

Plutonium Isotopic Decay Daughters (a)

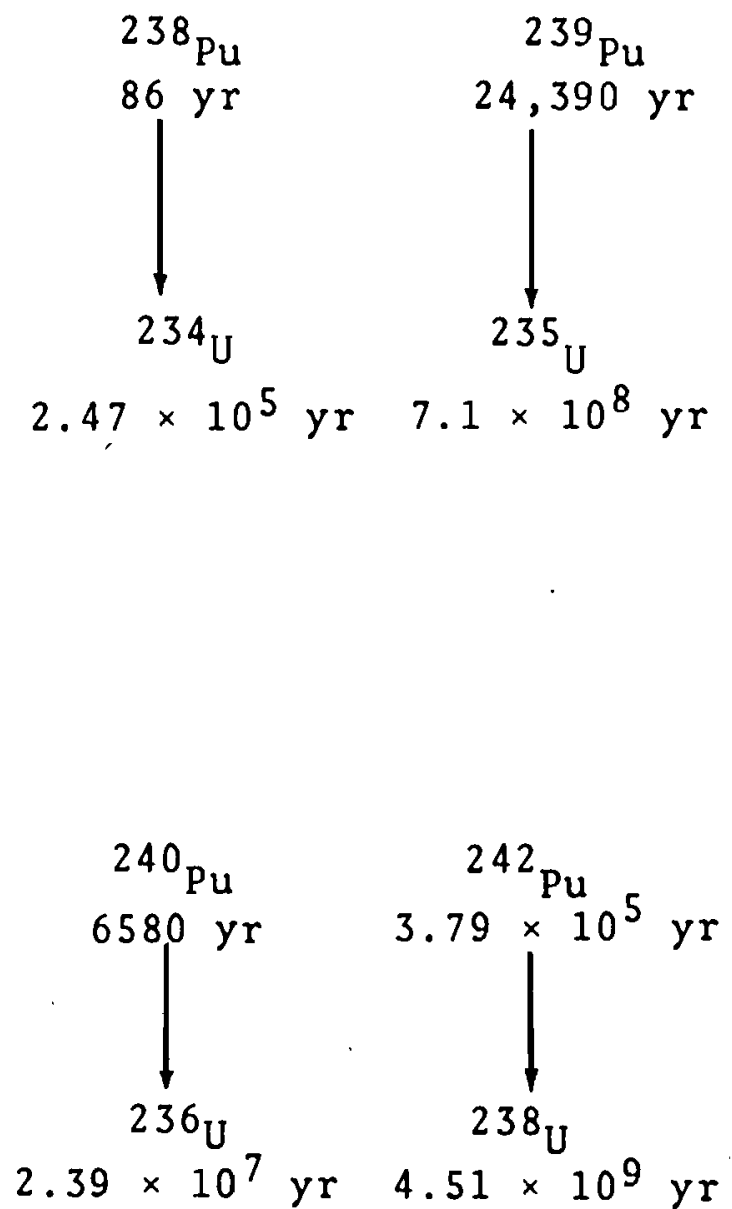

$236 \mathrm{Pu}$

$2.85 \mathrm{yr}$

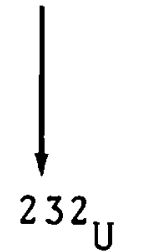

$72 \mathrm{yr}$

$2.39 \times 10^{7} \mathrm{yr} 4.51 \times 10^{9} \mathrm{yr}$
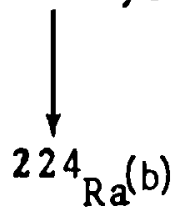

3.64 day

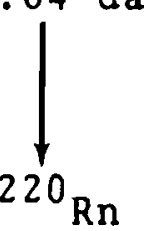

$55.3 \mathrm{sec}$
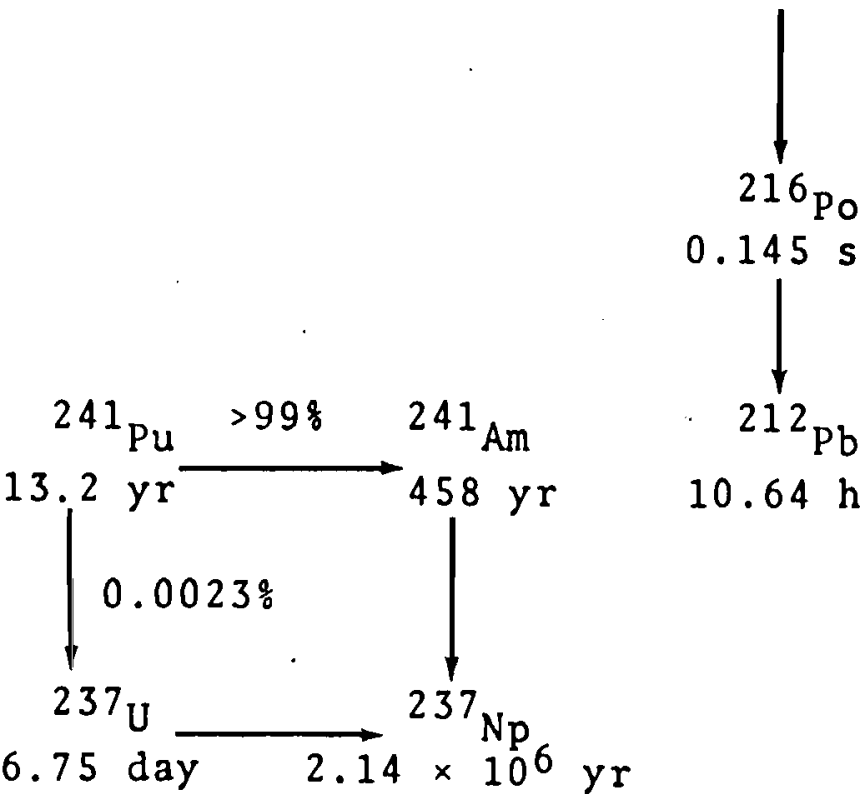

$0.145 \mathrm{sec}$
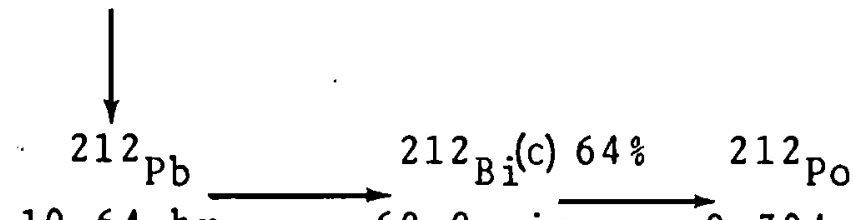

$10.64 \mathrm{hr} \quad 60.0 \mathrm{~min} \quad 0.304 \mu \mathrm{s}$

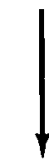

208

$36 \%$

$3.10 \mathrm{~min}$

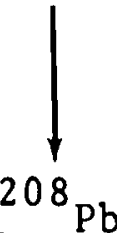
$208 \mathrm{~Pb}$

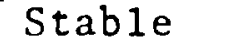

\footnotetext{
(a) From B!!!L-1259 - reference 12.

(b) The codes include aammas from Po-216 and $\mathrm{Rn}-220$ with this isotope.

(c) The codes include aammas from $\mathrm{Po}-212$ and $\mathrm{T} 1-208$ with this isotope.
} 
Figure 3

View of Plutonium Hood in Configuration Used

For Experimental Neutron Dose Rate Measurements

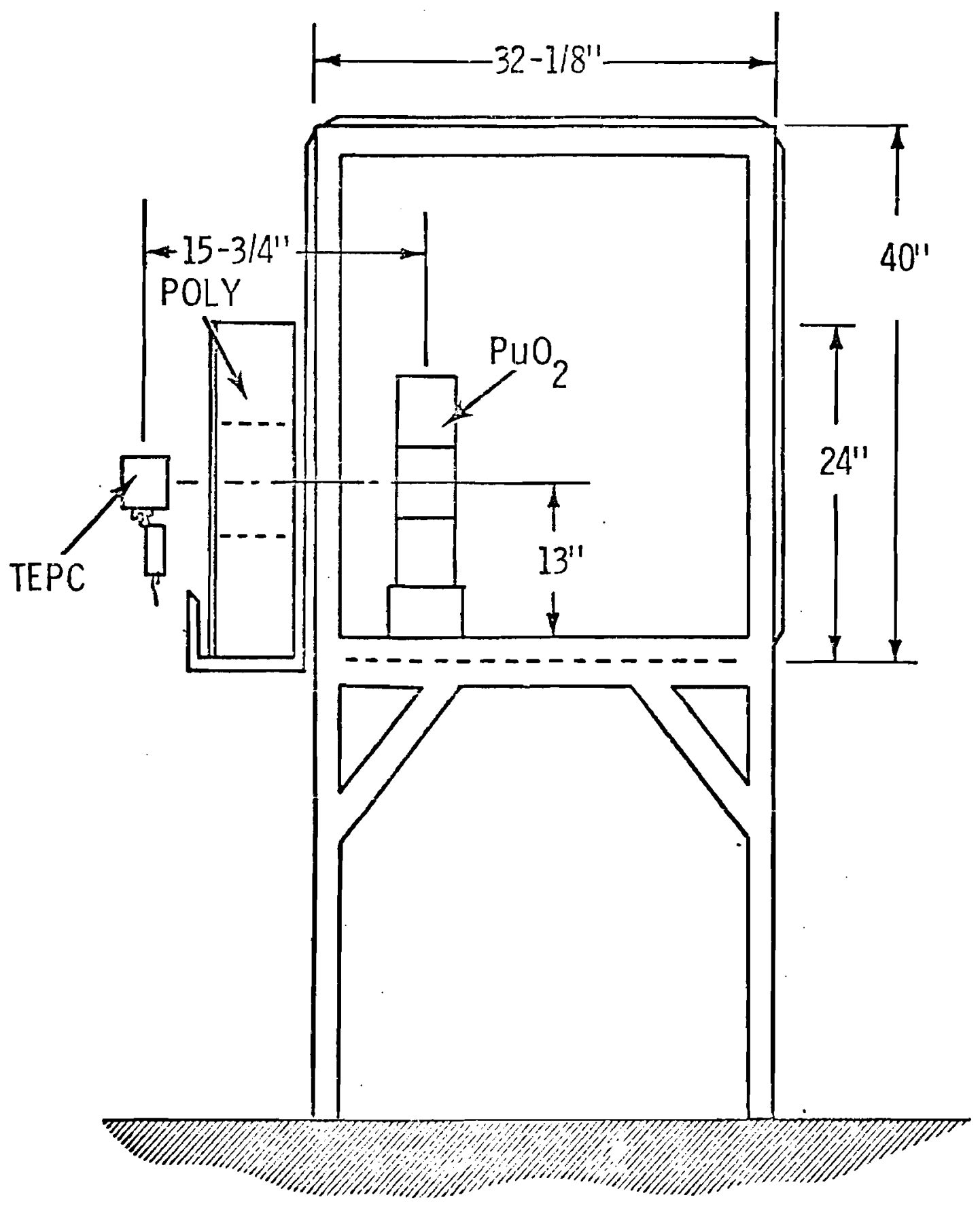


Figure 4

Glovebox Line As Modeled By the BMC-MG Code ${ }^{(a)}$

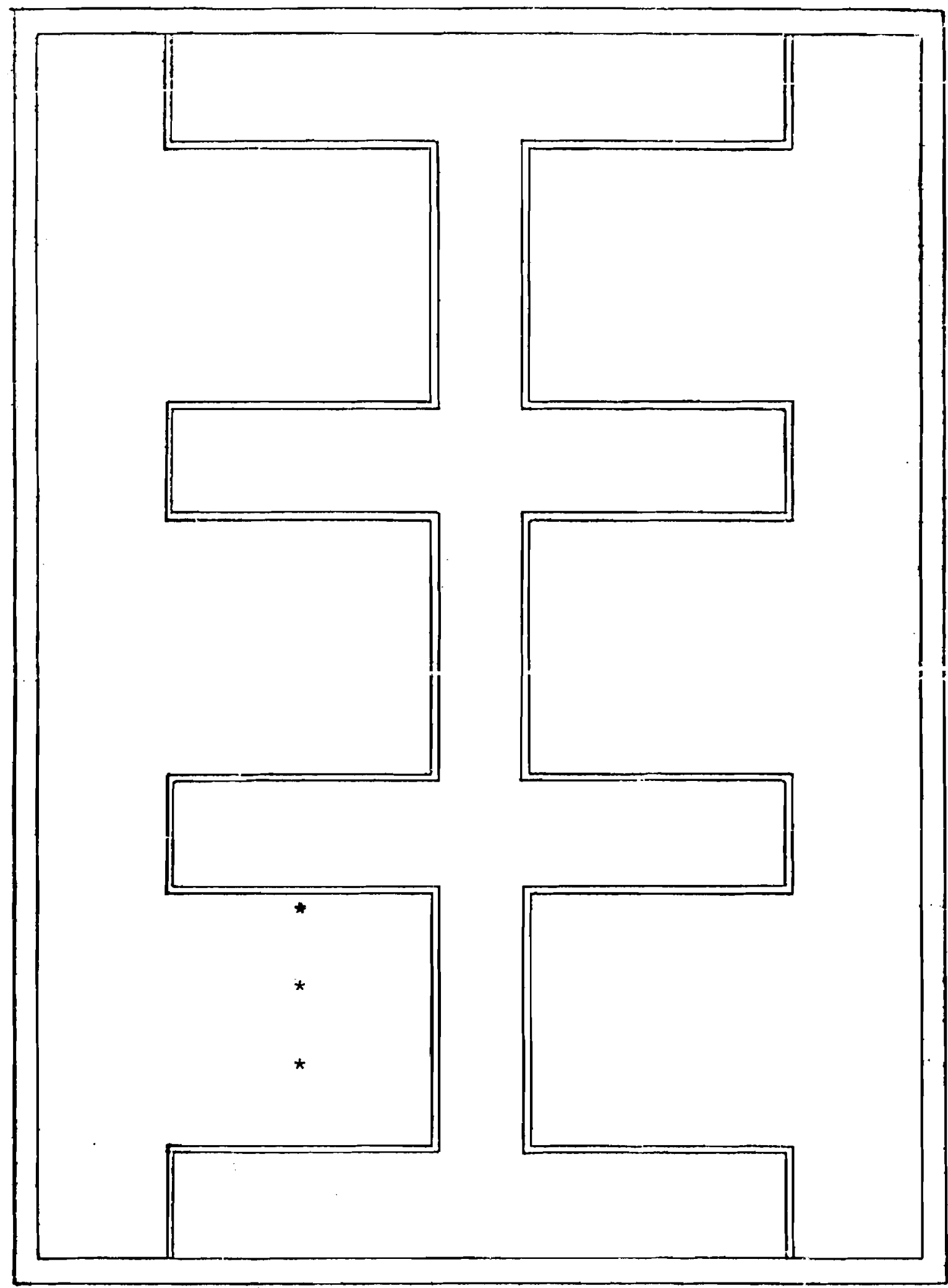

* Detector point locations. 
Figure 5

Section of Glove Box Line

As Modeled By The BMC-MG Code
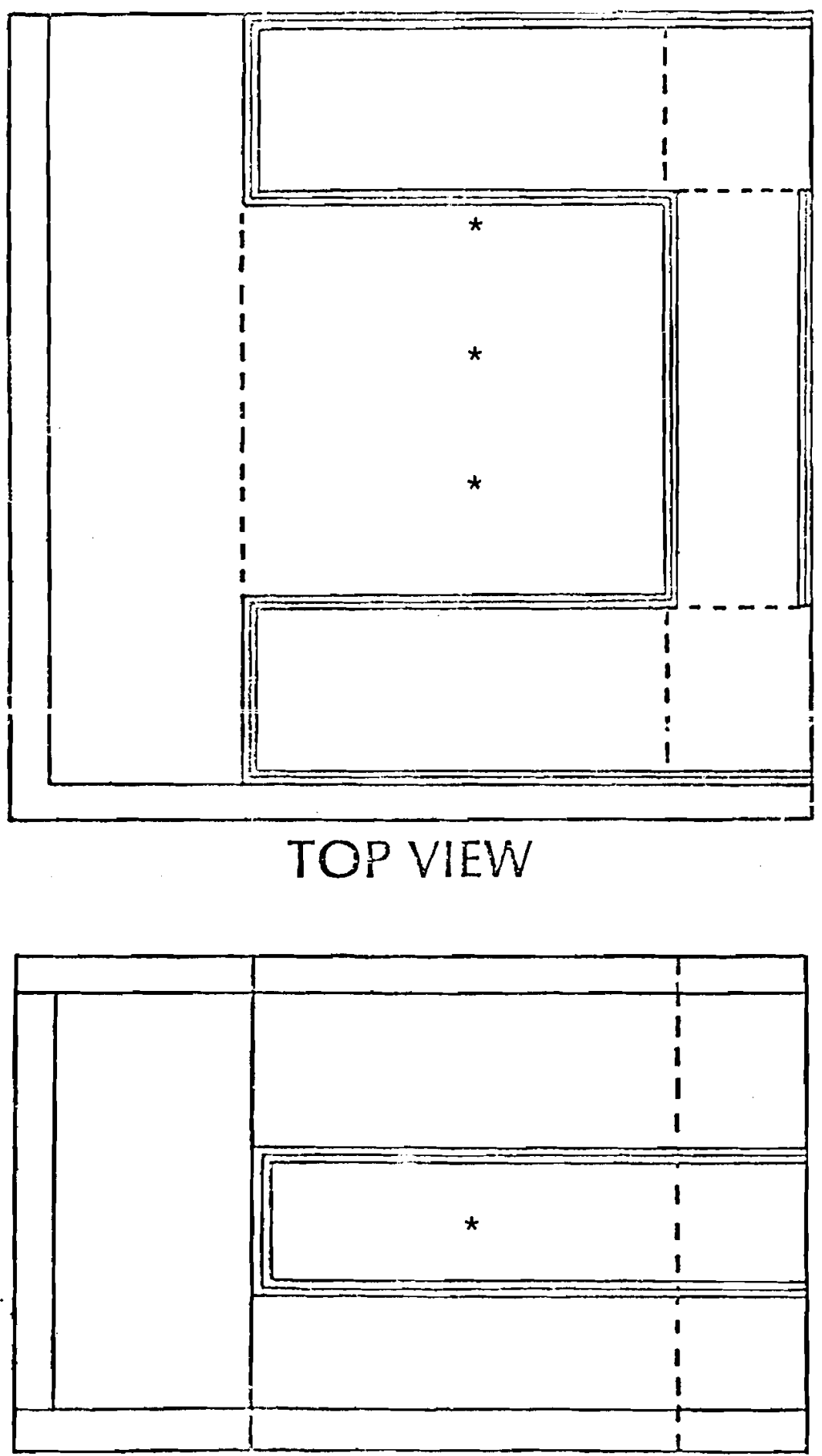

* Detector point locations 


\section{Figure 6}

Relative Neutron Dose Rate Versus Distance

From Source in A Glovebox Line

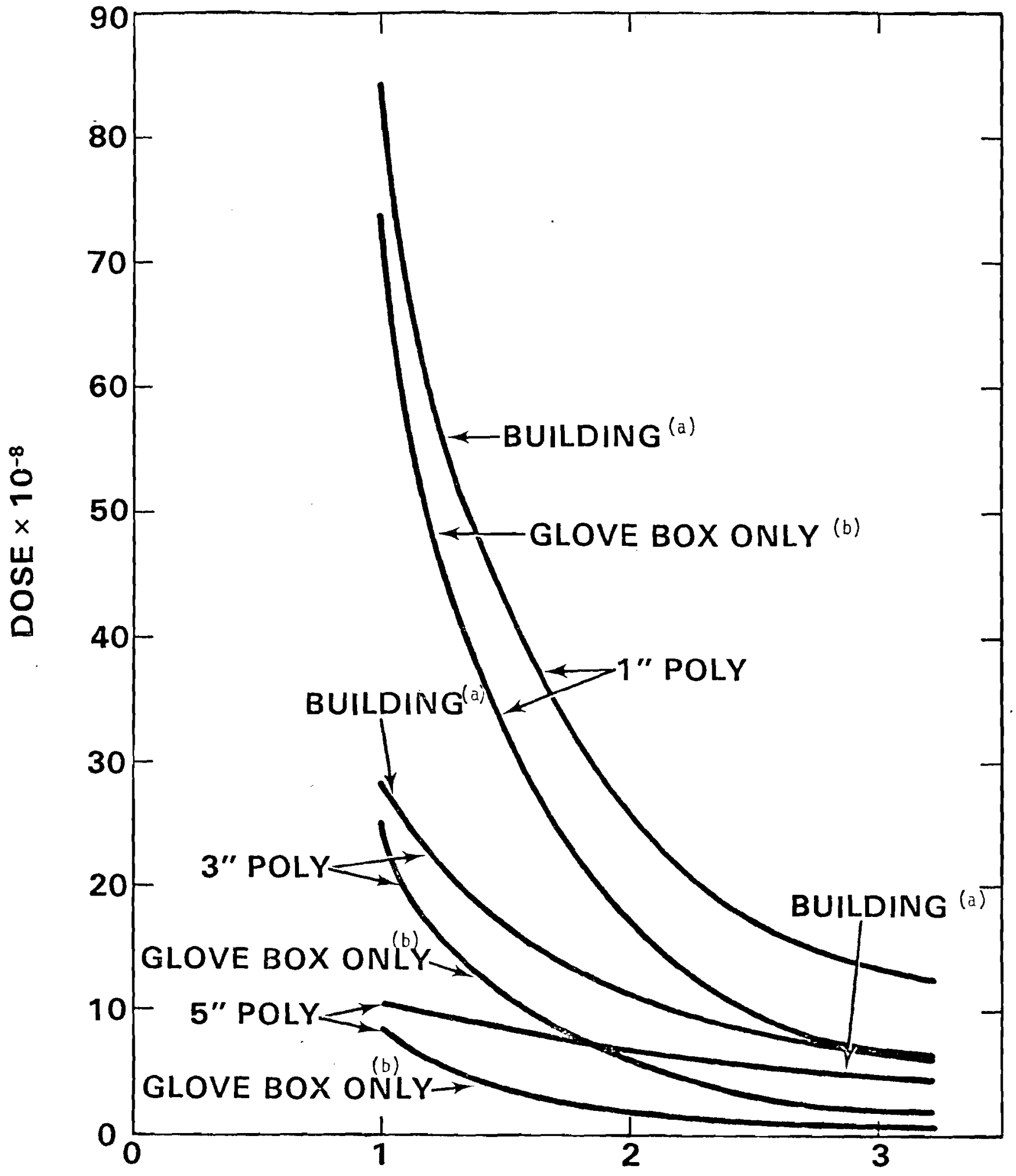

DISTANCE FROM SOURCE $\times 32$ INCHES

(a) Includes wall and floor scattering.

(b) Does not include wä1l and floor scattering. 
Figure 7

Gamma Dose Rate Measurement Experiment

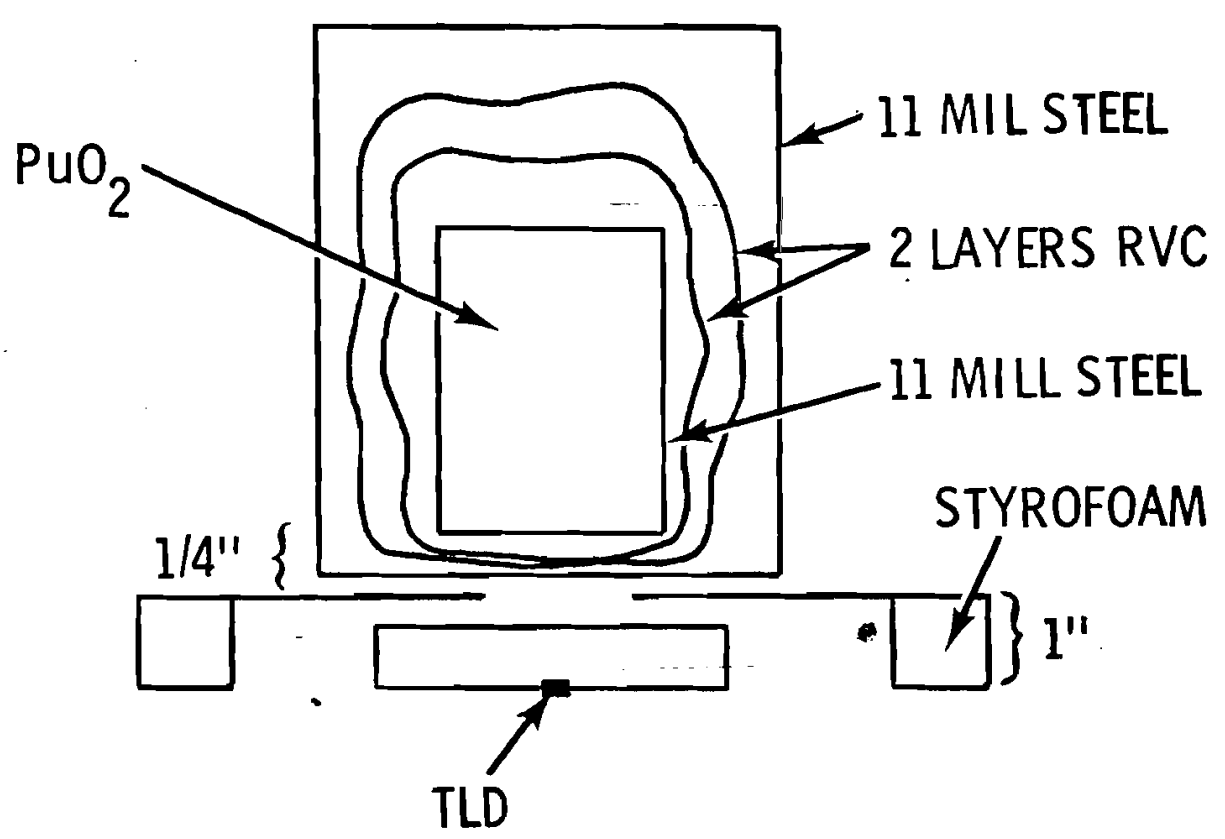


APPENDIX A 


\section{TABLE OF CONTENTS}

Page

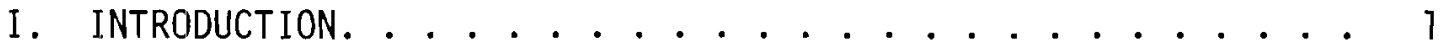

I I. THE BMC-MG MONTE CARLO ROUTINES . . . . . . . . . . . 4

III. THE MAJOR BMC-MG MONTE CARLO ROUTINES . . . . . . . . 15

IV. THE BMC-MG CODE: ORGANIZATION AND LAYOUT . . . . . . . . 28

V. THE BMC-MG OUTPUT ROUTINES. .................. 32

VI. THE BMC-MG INPUT ROUTINES . . . . . . . . . . 35

VII. DESCRIPTION OF CARD INPUT TO THE BMC-MG CODE. ....... 41

VIII. SAMPLE INPUT. .................... 45

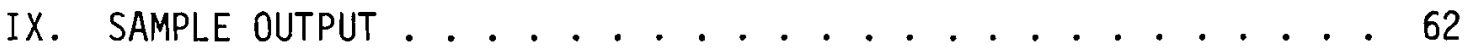




\section{BMC-MG: The Battelle Monte Carlo Code - Multi Group Version}

D. H. Thomsen, T. M. Traver and M. G. Zimmerman

\section{INTRODUCTION}

A. PHILOSOPHY AND HISTORY OF THE DEVELOPMENT

There are many neutronics problems encountered in reactor design studies, shielding, dosimetry studies, etc., which can not be adequately solved using the presently developed diffusion and/or transport theory methods. Moreover, experimental solutions to certain problems are either not feasible or practical. The Monte Carlo method is a powerful tool for solving these classes of problems because it provides a reliable calculational technique with a minimum of approximations.

The BMC-MG code is a multigroup version of the Battelle Monte Carlo (BMC). As the BMC code began being more in demand for shielding use, modifications were found desirable. In order to increase the flexibilitv for running neutron and gamma transport calculations the cross-section input was converted to a multigroup format. Also, a point detector capability was added. Other options have also been added to improve the codes efficiency. The major options include new biasing and importance weighting procedures. This is the version of the code developed for use in the calculational system. These changes are what make up the BMC-MG version of the BMC code. 
B. GENERAL FEATURES OF THE BMC CODE

The Battelle Monte Carlo Code - Multigroup Version (BMC-MG) is a general purpose Monte Carlo code and is primarily designed for use in shielding calculations and criticality calculations.

The neutron flux, isotopic reaction rates, group-averaged cross sections, and leakages are calculated in three-space dimensions and over any energy range. The cross sections are input in either the ANISN or the DTF format.

The BMC code geometry routines can handle any region which can be enclosed by a set of boundaries of the general form

$$
A\left(x-x_{0}\right)^{2}+B\left(y-y_{0}\right)^{2}+C\left(z-z_{0}\right)^{2}-K=0 .
$$

Provisions are included for seven special forms of the above equations including planes, cylinders and spheres. Also, spectal region geometry routines may be used; for example, one for a rectangular lattice of clad fuel rods has al ready been coded.

Statistics for the values calculated are obtained by making a series of calculations on equal sized sets of neutron histories called "batches" and averaging the results from each batch. The initial space-group-angle coordinates for each neutron of a batch are either picked from a random source distribution or from the fission particles produced by the previous batch.

Neutron absorption is accounted for by reducing the weight of the neutrons at each collision. When the weight is reduced sufficiently the neutrons are terminated by Russian Roulette. A special method of termination is available for thermal neutrons; that is, only part of the 
neutrons entering the thermal energy range are followed. The results are tallied into a second tally block. The results of the second tally set are then multiplied by the correct ratio and added to the complete tally set. This method allows a control on the relative amount of time spent calculating in the thermal range to that spent in the ep ithermal range.

The results calculated by the Monte Carlo routine are printed out by energy group by the output routine. The values obtained, along with their standard deviations, are the region-energy fluxes, the isotopic reaction rates and flux-averaged cross-sections, cell reaction rates, and leakages.

The BMC-MG code is coded in FORTRAN (95\%) and COMPASS (5\%) for use on the CYBER 74 computer system. Compatible cross-section libraries for neutron, gamma, and combined neutron-gamma cross section sets are available.

C. THE BMC CODE PACKAGE

The BMC code is made up of three segments or overlays comprised of the input routines, the Monte Carlo routines and the output routines. The input routines use card input and the CCT library tape to prepare the data needed by the Monte Carlo and output routines. The Monte Carlo routines are then loaded and the Monte Carlo calculation performed. The output routines are then used to process and print, the Monte Carlo output. Statistics are also calculated for the various output values. 
Section II of the report describes the theories and techniques which are used in the Monte Carlo calculation. In Section 1II, some of the major Monte Carlo routines are described. The output routines, and the problem input are discussed in following sections.

\section{THE BMC MONTE CARLO ROUTINES}

A flow diagram is shown in Figure 2 which describes the sequence of events in following the histories of a "batch" of neutrons. This is the path followed if importance weighting is not being used. The BMC code uses a beam type instead of a particle type Monte Carlo technique. It starts each tract with a beam. Carried along with the beam is one or more neutrons. The beam is used for tallying the flux, leakage, and point detector reaction rates. The neutrons are used for producing collisons. The beam strength is reduced by the negative exponential of the mean free path traveled between each boundary. A beam is followed until all of the neutrons have either collided or leaked. It can then be terminated either by leakage or by Russian Roulette.

A neutron beam is obtained by subroutine GETN. The neutron is then 10cated and the distance to the next boundary is calculated by subroutine LOCPRT which also supplies the material and tally region numbers. Subroutine MFPTH obtains the mean free path for this material. The distance-to-collision is compared to the number of mean free paths to the boundary. If a collision occurs, subroutine CQLLSN is called to perform the collision mechanics. COLLSN checks to see if a fission occurred and stores the fission neutrons in the fission bank. It uses the probability of nonabsorption to determine if a new scattered neutron will be produced. It then determines the 


\section{FIGURE 1}

THE BMC-MG CODE PACKAGE

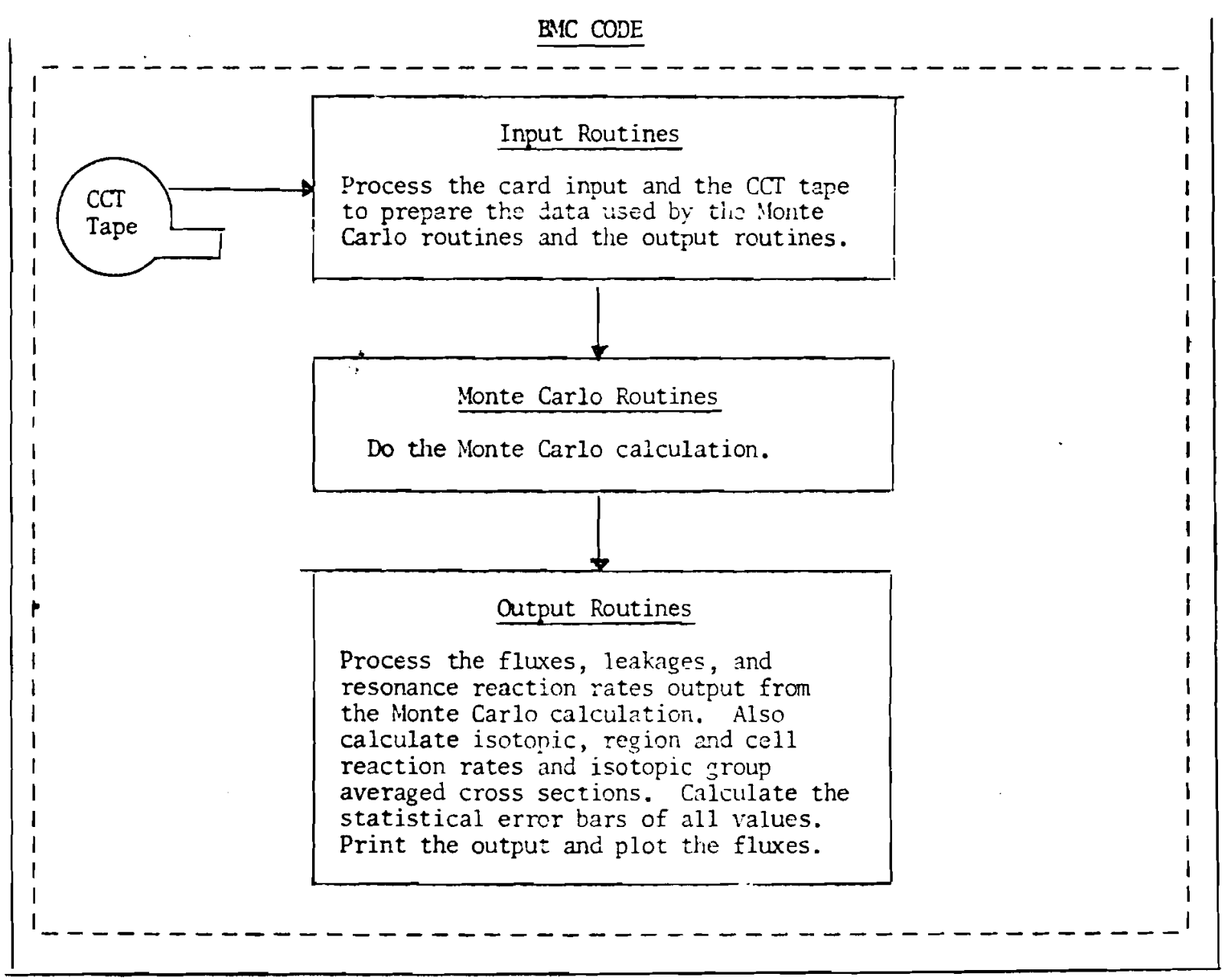


FIGURE 2

GENERAL FLOW DIAGRAM OF THE MONTE CARLO CODE

FOR NEUTRONS. IMPORTANCE WEIGHTING NOT BEING USED.

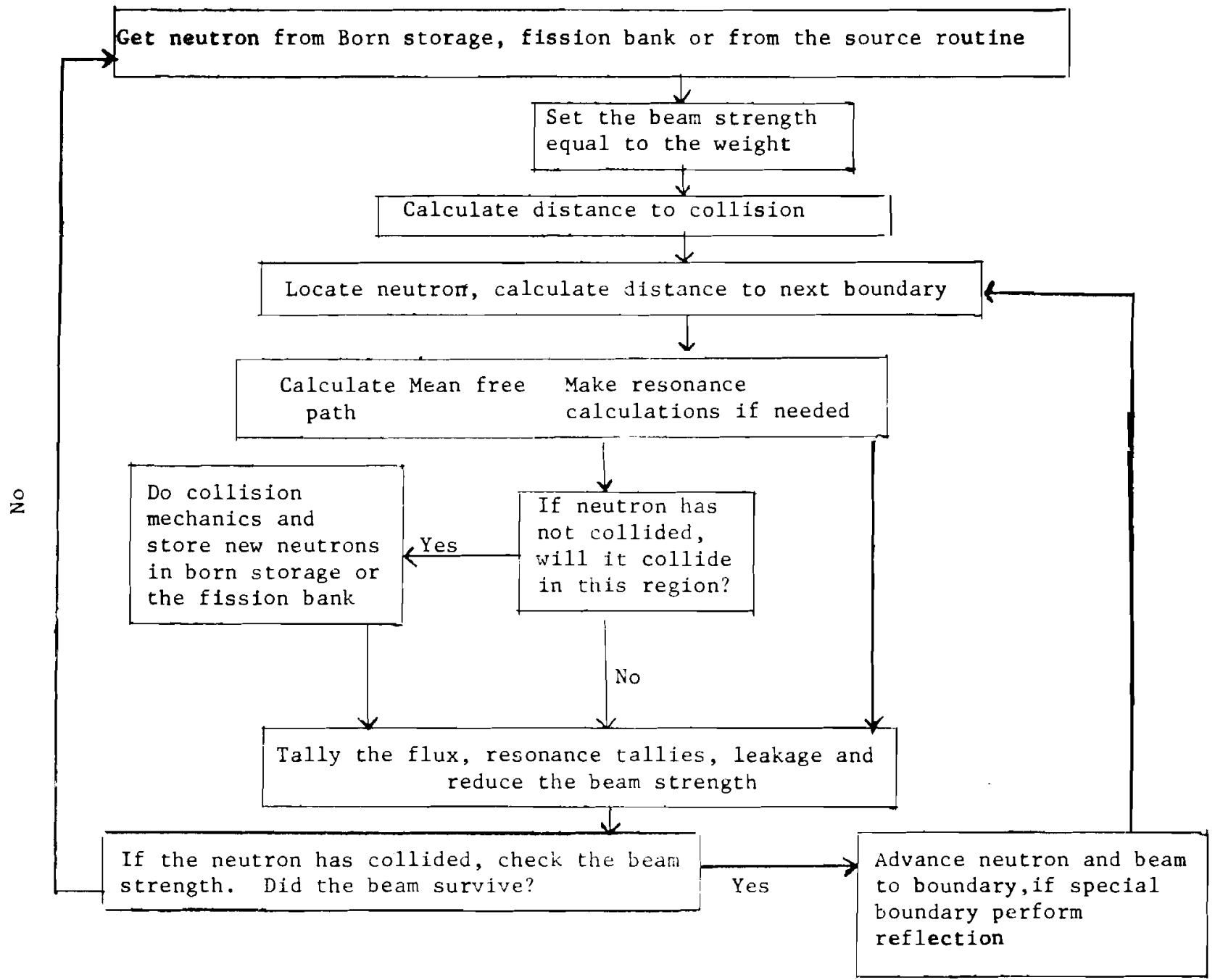


scattering nuclide and scattering event. A new direction and energy group are calculated and the scattered neutrons are stored in born storage.

Subroutine FXTLY is called to tally the flux, leakage, and resonance tallies. The beam strength is adjusted for the distance traveled in the region and tested to determine if it should be followed into the next region. The beam is then advanced to the next boundary where it crosses into the next region or is reflected. Subroutine LOCPRT is called agair: and the process is repeated. Note that each beam is followed until it is terminated by leakage or by Russian Roulette.

The flow of the calculation is altered slightly if region and energy importance weighting is being used. The main difference is that the calculation-of-distance to collision is made after the mean free path is obtained and must be made again in each region.

\section{A. TREATMENT OF NEUTRON ABSORPTION}

Neutron absorption is accounted for by reducing the weight of a neutron by the absorption probability at each collision. The reduction is done bv multiplying the weight before the collision by the non-absorption probability (Pna) for the collision. This process can be written as $W t^{\prime}=W t x$ Pna. The nonabsorption probability can be defined as the total scattering neutron production divided by the total cross section or

$$
\text { Pna }=\frac{\sum_{S}}{\sum_{T}}
$$

It is seen that no matter how many collisions the neutron suffers, the weight will never become zero. There has to be some way of killing the neutrons with low weight while conserving the total neutron weight. 


\section{Terminating the Neutron History}

Several obvious ways of terminating a neutron history are: let it escape, let it slow down past a lower cut-off energy, or to kill it when the weight is reduced below a certain minimum. The second method works fine for calculations of nonthermal parameters. The last method works for some problems but it does not conserve the total neutron absorption. The BMC code can use any of the above three methods to kill a neutron or it can use two other methods which are designed to statistically conserve the absorption. The two other methods are Russian Roulette and "weight ratioing".

Russian Roulette. Russian Roulette, as the name implies, uses chance to determine if a neutron survives. Given a minimum weight $\left({ }^{W t} \cdot \min \right)$ and a survival weight ( $t_{s}$ ), Russian Roulette is performed by picking a random number $(\xi)$ between 0 and 1 and if the ratio of the neutron weight to the survival weight is greater than the random number, then the neutron is given the survival weight. Russian Roulette is only played if the weight has been reduced below the minimum weight. This can be written as

$$
\begin{aligned}
& \text { if } w t<W t_{\min } \text { and if } \xi \leq \frac{W t}{W t} \text { then set } w t=W t_{s} \\
& \text { but if } \xi>\frac{W t}{W t} \text { then set } W t=0 \text { and } k i 11 \text { the neutron. On the average }
\end{aligned}
$$
with a large number of samples, the weight will be preserved by this method.

The RBU Monte Carlo code and some other codes do not use the weight reduction method to account for absorption; instead they sample a random number against the nonabsorption probability at each collision. This is the same as setting $W t_{\min }$ and $W t_{s}$ both equal to unity. 
Particle Splitting. A value $W t_{\max }$ may also be input. If the particle has a weight greater than $W t_{\max }$ then the particle is split into several particles using the equation $N_{p}=E W t / W t_{\max }+\xi \xi$ where $\xi$ is a random number and $E \quad \exists$ is the greatest interger.

Weight Ratioing. For very thermal systems, it may take a very large number of collisions to kill neutrons by using Russian Roulette. This is because the weight reduction at each collision is very small (Pna $\approx 1$ ). Often this results in obtaining answers concerning thermal parameters which are statistically very accurate while the accuracy of the epithermal parameters is very poor. A method called "weight ratioing" was devised to allow control of the time spent in the thermal conpared to the epithermal calculations.

Weight ratioing uses two flux tally sets: one tally set for neutrons slowing down and one for neutrons which have been slowed down past an entry energy $\left(E_{t h}\right)$. The neutrons reaching the thermal tally range (second tally set) can have their energy increased above $E_{\text {th }}$ but the results are still tallied into the thermal tally set.

The way weight ratioing works is to tally the results of a neutron history into tally set 1 until it is slowed down into the energy group $E_{\text {th }}$. Russian Roulette is then played with each neutron so that $R_{t h}$ neutrons entering the thermal tally set are rejected for each one entering the thermal tally range. The ones which survive are followed and the results tallied into a second tally set. The final tallies are the sums of the values from the first tally set plus the values from the second tally set times a weighting. This ratio is the weight entering the thermal tally range divided by the weight lost by neutrons followed in the thermal tally 
range or approximately $1 /\left(1-R_{t h}\right)$. This method statistically conserves the neutron absorption and allows a way of controlling the statistics obtained for epi-thermal parameters relative to those obtained for thermal parame ters.

This method has not been thoroughly tested to determine the correct values of the different parameters involved or when it should or should not be used. However, it has been observed that a value for $E_{\text {th }}$ slightly higher than $6 \mathrm{kT}$ works fairly well. Figure 3 is a graph which is useful in trying to decide the values to use for $R_{\text {th }}$ and for Russian Roulette. It shows the number of collisions needed on the average to produce one absorption using Russian Roulette as a function of the average nonabsorption probability.

B. THE NEUTRON FLUX AND THE NEUTRON BEAM

The neutron flux at energy $E$ integrated over volume and energy is the total neutron track length in the volume, or

$$
\phi_{\text {if }}=\int_{V_{f}} \int_{E_{i}} \phi(E, V) d E d V=\sum_{n=1}^{N} \ell_{n} W t_{n}
$$

where $V_{f}$ is the volume of region $f, \ell_{n}$ is the track length and $W t_{n}$ is the weight of the nth neutron in region $f$ and energy group $i$. Note that the reaction rate for the $\underline{k}$ th event if $R_{i, f, k}=\Sigma_{i, f, k} \phi_{i f}$ where $\Sigma_{i, f, k}$ is the macroscopic cross section.

Instead of tallying the track length of individual neutrons the BMC code tallies the estimated track length for a beam of neutrons going in the same direction as the individual neutron. The flux contribution to a region and energy group can be written as 


\section{FIGURE 3}

THE RELATIONSHIP BETWEEN THE NUMBER OF COLLISIONS AND THE NONABSORPTION PROBABILITY AS A FUNCTION OF THE MINIMUM AND SURVIVAL WEIGHT

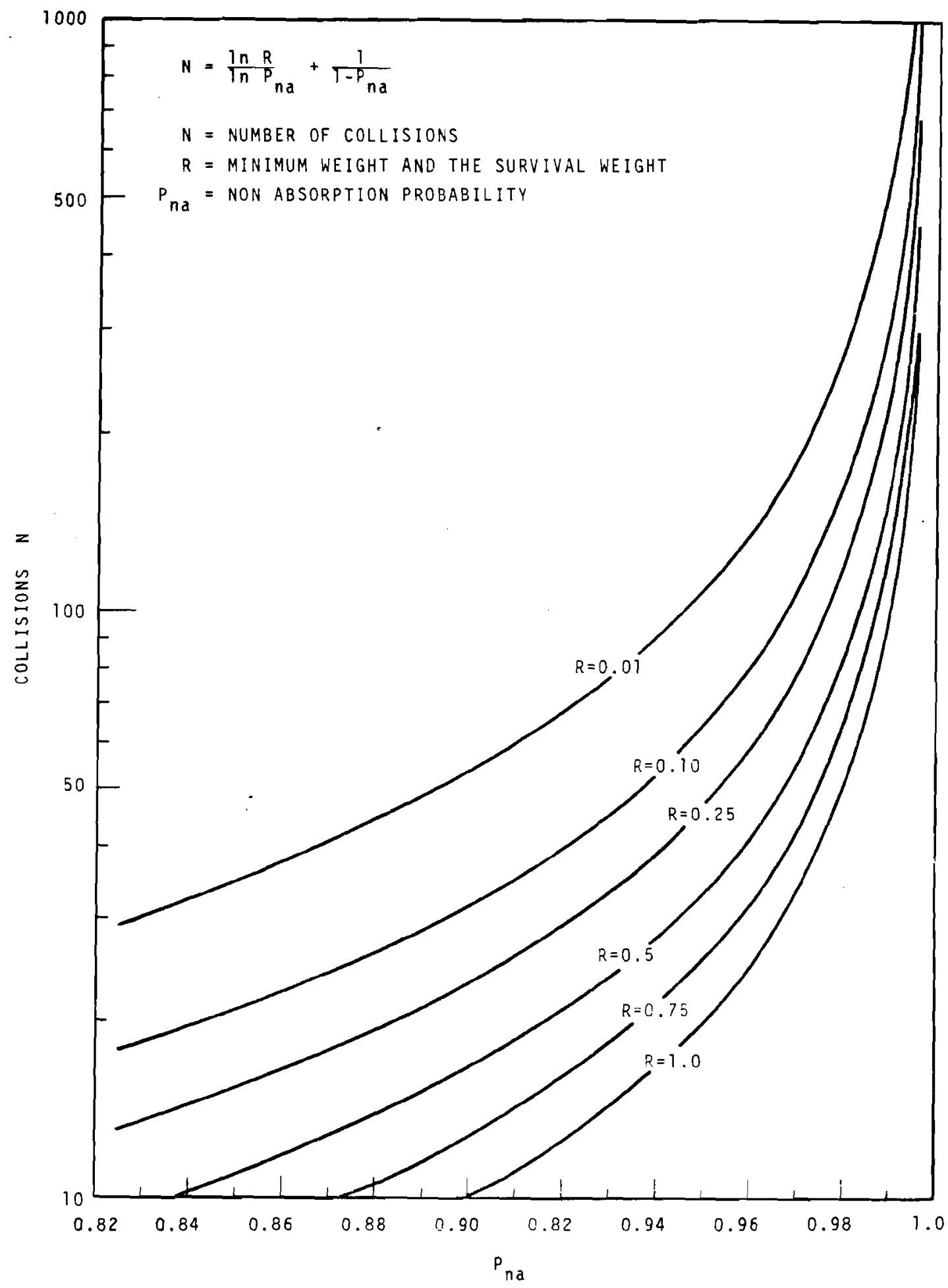




$$
\phi_{\text {if }}=\sum_{n=1}^{N} \lambda_{\text {if }} \pi_{n}\left(1-e^{-S_{b n} / \lambda_{i f}}\right) \text { where } \lambda_{\text {if }} \text { is the mean free path }
$$

in the $i$ th energy group of region $f, S_{b n}$ is the distance to the out side boundary of the region for the nth beam, and $\pi_{n}$ is the beam strength at the beginning of the nth flight of the beam. The beam strength is set equal to the weight of the neutron at the start of the neutron path. It is then diminished by the factor $\mathrm{e}^{-\mathrm{S}_{\mathrm{bn}} / \lambda}$ if at each boundary crossing. Note that the neutron is followed until it has a collision or leaks from the cell while the beam is followed even after the collision. If the neutron leaks then the beam is also terminated; otherwise the beam is terminated by using Russian Roulette.

\section{LEAKAGE ESTIMATE}

The leakage tallied by the beam crossing a boundary is just the beam strength, $\pi$, at the boundary. The leakage is tallied as a function of energy group.

D. IMPORTANCE WEIGHTING

Provisions have been added to the BMC multigroup code for both zone and energy importance weighting. Zone and energy importance weighting provides a method for spending more time in regions (zones) or energy groups of greater importance while reducing the time spent in regions or energy groups of low importance. When importance weighting is used the number of neutrons in the beam and the weight of each neutron is modified when the beam passes between regions or energy groups of different importance. 
If region 1 or energy group 1 has importance ZWT 1 and region 2 or energy group 2 has importance $\mathrm{ZWT}_{2}$ then as a neutron beam of $\mathrm{PN}_{1}$ neutrons and weight $W_{1}$ passes to region 2 or downscatter to energy group 2 the new weight and number of neutrons become

$$
W t_{2}=\frac{2 W T_{1}}{2 W T_{2}} \cdot W t_{1}
$$

and

$$
\mathrm{PN}_{2}=\left[\frac{Z W T_{2}}{Z W T_{1}} \cdot \mathrm{PN}_{1}+\S \neq\right.
$$

where $\S$ is a random number and [] means greatest integer. Both importance weighting functions can operate at the same time.

E. NEUTRON BATCHES

A BMC calculation is comprised of a set of calculations called batches. Each batch contains the same number of neutron histories. The neutron histories can start as neutrons born at fission or as source neutrons put into the system using any desired source distribution. The results from the batches are then averaged to obtain the final calculated values.

F. FISSION DESCENDANT OR DIRECT SOURCE PROBLEMS

The BMC code can be used in three problem modes. One mode, the fission descedant problem, allows fission neutrons to be born as a resuit of collisions. These fission progeny are stored and used as starting neutrons for the next batch. The second mode, called the direct source problem, obtains all starting neutrons from the source routine. In this 
case, there is no need to save the fission neutrons, so the fisston reaction is not sampled. The third mode is a combination of the first two modes. In thismode the initial neutrons are obtained from the source routine but fission neutrons are also allowed as a result of collisions and used as part of the source. The direct source and fission neutrons may have different spectra.

\section{G. THE NEUTPON POSITION-DIRECTION-VELOCITY PARAMETERS}

Associated with each neutron is a set of parameters which describe its position, direction, and energy group. The position is described by the position vector $x, y, z$ where the units are in centimeters, and an index $I_{h}$ which tells which geometrical region the neutron is in. The direction is defined by the three normalized direction cosines $\alpha, \beta$, and $\gamma$. The energy group is defined by the index, $I_{V}$.

The neutron beam also has several parameters associated with it. They are the weight, $W t$, the number of particles, $\mathrm{Np}$, and the beam strength $(\pi, B P I)$.

\section{H. ENERGY GROUP STRUCTURES}

The energy range studied by the $B M C$ code is dependent only on the energy range of the cross-sections. The neutron energy-velocity distribution is modeled in multigroup form. There are two different energy group structures. They are the input multigroups and the broad groups.

The cross sections are input as group averaged multigroup cross-sections. The fluxes are also tallied for each multigroup.

Most calculations with the BMC code have been made using 27 neutron energy groups and 16 gamma energy groups. 


\section{CODE CHECK TALLIES}

A set of values are tallied as the Monte Carlo calculation is being made which serve as code check tallies. They are a set of values which are not needed as output, but serve as a means of checking the performance and characteristics of the Monte Carlo code. The code check tallies are obtained directly from the neutron histories and are not results obtained using the flux estimator. As a note of caution, the answers obtained using the code check tallies are usually not as accurate as those obtained using the flux estimators.

The code check tallies include such things as the number of initial fission or source neutrons, the number and weight of neutrons to and from collision, the number of neutrons leaking from the system, the loss of beam strength and weight by application of Russian Roulette, and the number and weight of neutrons entering, followed in, and leaving the thermal tally range, etc.

The code check tallies are written out at the completion of each Monte Carlo batch. Average values with associated statistical errors are also written by the output routines.

\section{THE MAJOR BMC MONTE CARLO ROUTINES}

This section will describe the functions of each of the major routines and sections of the BMC Monte Carlo Code. The order described in Figure 1 will be followed. First the random number routines will be discussed briefly. 
A. THE BMC RANDOM NUMBER ROUTINES

The random number routines originally used in the BMC code are those used in the IBM-7090 version of the 05R Monte Carlo code. They were converted to SLEUTH for use on the Univac-1108 computer. These routines have now been converted to FORTRAN and use the COMPASS routine RANF to generate random numbers. Table 1 gives the names of the random number routines along with their distribution.

\section{TABLE I}

RANDOM NUMBER ROUTINES USED IN THE BMC CODE

Fortran Calling Statement

$\mathrm{X}=\mathrm{RNF}(\mathrm{IDUMMY})$

$X=\operatorname{SRNF}($ IDUMMY $)$

$X=R N L \theta G(I D U M M Y)$

CALL AZIRN(SIN,COS)

CALL POLRN(SIN,COS)

CALL RNCES (ALPHA, BETA, GAMMA)
Random Number Distribution

Uniform distribution on the interval $0 \leq x \leq 1$.

Uniform distribution on the interval $-1 \leq \mathrm{x} \leq 1$.

Exponential distribution $\mathrm{P}(\mathrm{x}) \mathrm{dx}=\mathrm{e}^{-\mathrm{x}} \mathrm{dx} \quad 0 \leq \mathrm{x} \leq \infty$.

The sine and cosine of $\phi$ where $\phi$ is uniformly distributed on the interval $0 \leq \phi \leq 2 \pi$. A random azimuthial angle.

The $\sin$ and $\cos$ ine of $\theta$ where $\cos \theta$ is uniformly distributed on the interval $-1 \leq \cos \theta \leq 1$. A random polar angle.

An isotropic unit vector with direction cosines ALPHA, BETA, and GAMMA. 
B. GETN - ROUTINE TO GENERATE, SAVE, AND RETRIEVE NEUTRONS

The GETN subroutine is used to obtain source or fission neutrons or to retrieve collision neutrons. Other entries to GETN are used to store neutrons from fission or scattering.

1. Storage Blocks Used By GETN

GETN uses two storage blocks: born storage and the fission bank. Born storage is used to save the neutrons born from collision or slowed down while the fission bank is used to store the neutrons from fission. The neutron parameters which are saved in born storage are $x, y, z, \alpha, \beta, \gamma$, $I_{v}, I_{h}$, and $W t$. Only spatial parameters $x, y, z$, and $I_{h}$ are saved in the fission bank. When a neutron is taken from the fission bank the other parameters are obtained by GETN using an isotropic distribution for the direction cosines, and a random fission distribution for the velocity. The built in fission distribution is presently that for uranium-235; however, the fission distribution can be specified via input.

When GETN is called, born storage is checked first. If it is empty then the fission bank is checked or the source routine is called. If the broad group and/or batch is finished, the return from GETN is via the call list.

\section{The source Routine}

The source routine is used to pick the initial parameters for the neutron histories. If the calculation is a fission descendant problem, the source routine will only be used for the first batch and only the spatial parameters will be picked. For a direct source problem, all the parameters are generated by the source routine. The user can write a source routine for a particular problem or use the one built into the BMC code. 
The source routine is very versatile. It allows the selection of the source spatial distribution using combinations of point, equal volume, $\operatorname{cosine}\left(\frac{\pi}{3} \frac{r}{R}\right)$, Jo $\left(2.405 \frac{r}{R}\right)$ and $(\operatorname{Sin}(t r) / \pi r$ distributions. The energy spectrum can be picked from built in source spectra from fission and various $(\alpha, n)$ interactions or the spectra can be input via cards. The angular distribution can vary from isotopic to nondirectional and can be biased using several built in bias functions.

C. CALCULATION OF DISTANCE TO COLLISION

The distance to collision in units of mean free paths is obtained by sampling from the distribution $P(\lambda) d \lambda=e^{-\lambda} d \lambda$.

D. LOCPRT - THE BMC GEOMETRY ROUTINE

The geometry routine, LOCPRT, is used to locate which region a neutron-beam is in and to calculate the distance to the next boundary. A control (IBFN) is used to guide the calculation. If IBFN $=1$ the neutron is a source, fission, or collision neutron. If IBFN $=2$ the neutron-beam has just crossed the boundary into another region. If IBFN $=3$ the neutron-beam has just been reflected back into the same region. LOCPRT also contains the boundary reflection routines for reflecting a neutronbeam either isotropically or with a mirror image reflection.

\section{Region Definition}

Regions are identified by an index $I_{h}$. For each region one specifies the number of boundaries $\left(\mathrm{N}_{\mathrm{b}_{h}}\right)$, an index $\left(\mathrm{L}_{\mathrm{s}_{\mathrm{p}_{h}}}\right)$ telling if this is a special 
region, the number of the material $\left(M_{h}\right)$ contained in the region, and the tally region $\left(f_{h}\right)$ that this region is part of.

For each of the $\mathrm{N}_{\mathrm{b}_{h}}$ boundaries one specifies four quantities. They include the index of the boundary $\left(\mathrm{I}_{\mathrm{b}_{\mathrm{h}}}\right)$, a word (IGD) which contains two bits of information, and two indexes $\left(\mathrm{IQ}_{1}\right.$ and $\left.\mathrm{IO}_{2}\right)$ that te 11 which region to look in for the neutron if it leaves region $I_{h}$ across boundary $i_{b_{h}}$. Region $\mathrm{IQ}_{1}$ is first checked and if the neutron is not in that region a complete region search is made starting in region $\mathrm{IQ}_{2}$.

The two bits of information in IGD are $G_{b}$ and $D_{b} \cdot G_{b}$ is a sign that tells which side of the boundary the neutron is on to be inside of the region. $D_{b}$ gives the number of the leakage tally set to use if a neutron crosses this boundary. The way that $G_{b}$ and $D_{b}$ are combined in IGD is illustrated by the equations:

$$
G_{b}=\text { sign of IGD, } D_{b}=|I G D|-1 \text {. }
$$

If the value of $D_{b}>50$ then the neutron beam strength crossing the boundary will be tallied but the beam will not be treated as if it were leaking from the system.

The regions must be combined in a way so that a neutron can always be located. Also the regions should not overlap each other. It is not necessary to define all of space if the cell is surrounded by reflecting or leakage boundaries and all initial neutrons are inside the cel1.

\section{Special Regions}

The LOCPRT routine allows the use of special regions. Special regions are regions containing geometrical designs which are to be treated by a 
separate geometry routine. One special geometry routine for a rectangular lattice of clad fuel rods has already been written.

LOCPRT first finds the distance to the outer boundary of the special region. The special routine is then called to calculate the distance to the nearest boundary of the special regions.

3. Boundary Definitions

There are eight types of boundaries which can presently be used by the LOCPRT routine. The type of each boundary is specified by an index $I_{d}$. Another index, IOM ${ }_{b}$ is used to determine if the neutron will cross boundary $I_{b}$ or will be reflected from the boundary.

Each boundary defines a boundary function $r$. When $r=0$ the neutron is on the boundary. The sign of $r$ when the neutron is not on the boundary tells which side of the boundary the neutron is on. A point is said to be inside of a region if the product of $G_{b} \cdot r$ is negative for all the boundaries in the region.

Table II gives the boundary index, boundary function and description of the eight boundaries now included in BMC. It would also be easy to add other type boundaries to the code. 
TABLE II

BMC CODE BOUNDARY FUNCTIONS

$\begin{array}{lll}\frac{I_{d}}{1} & \begin{array}{l}\text { Boundary Function } \\ r \equiv x-x_{0}\end{array} & \begin{array}{l}\text { Description } \\ \text { Plane at } x=x_{0}\end{array} \\ 2 & r \equiv y-y_{0} & \text { Plane at } y=y_{0} \\ 3 & r \equiv z-z_{0} & \text { Plane at } z=z_{0} \\ 4 & r \equiv y-a x-b & \text { Plane on } y=a x+b \\ 5 & r \equiv x^{2}+y^{2}-R^{2} & \begin{array}{r}\text { Cylinder of radius } R \\ \text { centered on } z \text { axis }\end{array} \\ 6 & r \equiv\left(x-x_{0}\right)^{2}+\left(y-y_{0}\right)^{2}-R^{2} & \begin{array}{r}\text { Cylinder of radius } R \\ \text { centered at } x_{0}, y_{0}\end{array} \\ 7 & r \equiv x^{2}+y^{2}+z^{2}-R^{2} & \begin{array}{r}\text { Sphere of radius } R \text { centered } \\ \text { at the origin }\end{array} \\ 8 & r \equiv A\left(x-x_{0}\right)^{2}+B\left(y-y_{0}\right)^{2}+C\left(z-z_{0}\right)^{2}-k & \begin{array}{r}\text { General boundary function }\end{array}\end{array}$


Determination of $G_{b}$. Remember that for a particle to be inside of $a$ region that the product $G_{b} \cdot r$ must be negative for all of the boundaries of a region. To determine which sign to use for $G_{b}$ just pick a point inside the region and solve the boundary functions for the region boundaries. For spheres and cylinders, $G_{b}$ is positive inside and negative outside. For the $x_{0}, y_{0}$ and $z_{0}$ planes, $G_{b}$ is positive for the region below the plane and negative above.

\section{Sample Geometry Description}

A sample of the geometry description for a first flight collision probability calculation is given in Figure 4 . The boundary data and region data are also given.

\section{Calculation of Distance to Boundary}

The distance to boundary $\left(S_{b}\right)$ is calculated using the equations shown in Table III. For boundary types 5 to 8 the distance to boundary is obtained by solving the quadratic equation $f\left(S_{b}\right)-h S_{b}^{2}+2 e S_{b}+r=0$. Now apply Sturm's theorem to this equation to see if there are any roots between zero and infinity. The three Sturm's functions for the quadratic equation $f\left(S_{b}\right)$ are:

$$
\begin{aligned}
& f\left(s_{b}\right) \\
& f^{-}\left(s_{b}\right)=2 h S_{b}+2 e \\
& F_{1}=e^{2} / h-r .
\end{aligned}
$$


FIGURE 4

EXAMPLE OF GEOMETRY DESCRIPTION

FIRST FLIGHT COLLISION PROBABILITY CALCULATION

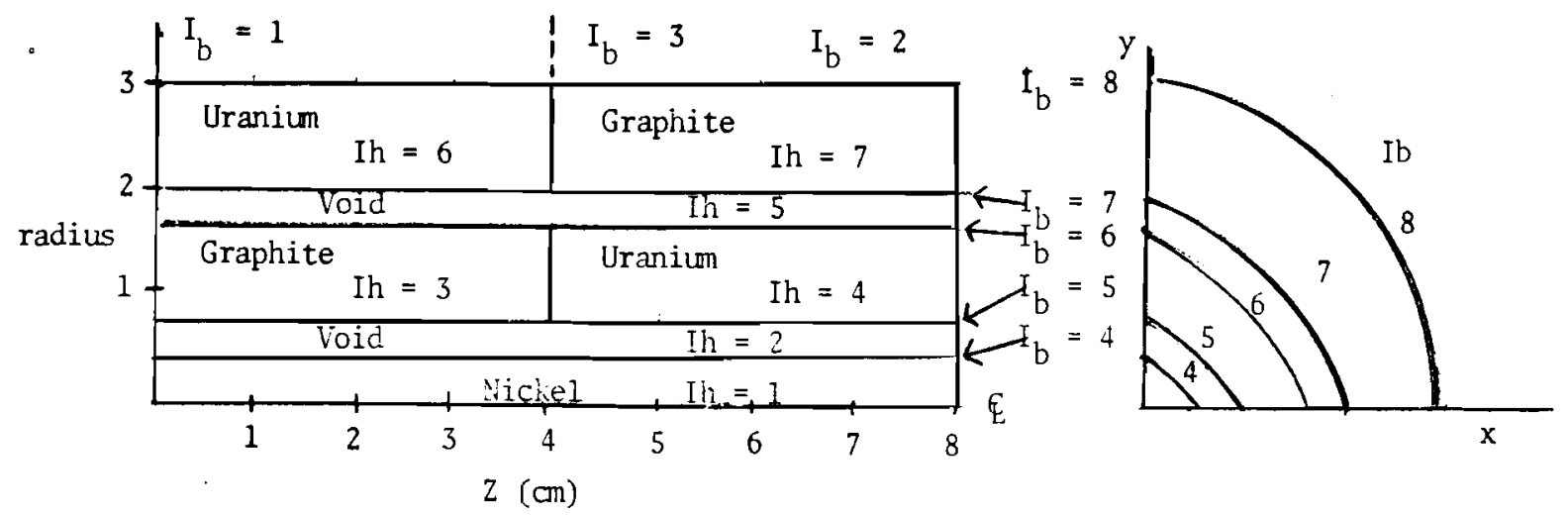

Material

$\begin{aligned} 1 & \text { Nickel } \\ 2 & \text { Graphite } \\ 3 & \text { Uranium } \\ -1 & \text { Interior Void }\end{aligned}$

Let the tally region number, $f_{h}$, be the same as the region number, Ih.

BOUNDARY DESCRIPTION

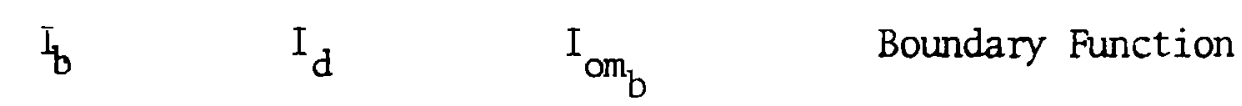

$\begin{array}{llll}1 & 3 & 3 * & r \equiv z-0 \\ 2 & 3 & 3 & r \equiv z-8 \\ 3 & 3 & 0 & r \equiv z-4 \\ 4 & 5 & 0 & r \equiv x^{2}+y^{2}-(0.5)^{2} \\ 5 & 5 & 0 & r \equiv x^{2}+y^{2}-(0.75)^{2} \\ 6 & 5 & 0 & r \equiv x^{2}+y^{2}-(1.75)^{2} \\ 7 & 5 & 0 & r \equiv x^{2}+y^{2}-(2.00)^{2} \\ 8 & 5 & 0 & r \equiv x^{2}+y^{2}-(3.00)^{2}\end{array}$

${ }^{*} \mathrm{I}_{\mathrm{om}_{\mathrm{b}}}=3$ for a mirror reflecting bourdary. 
FIGURE 4 (continued)

\section{REGION DESCRIPTION}

\begin{tabular}{|c|c|c|c|c|c|c|c|c|c|c|c|c|c|}
\hline $\mathrm{I}_{\mathrm{h}}$ & $\mathrm{N}_{\mathrm{b}}$ & & & $\mathrm{M}_{\mathrm{h}}$ & $f_{h}$ & IGD & $I_{b}$ & $\mathrm{Q}_{1}$ & $Q_{2}$ & IGD & $I_{b}$ & $\mathrm{Q}_{1}$ & $Q_{2}$ \\
\hline \multirow[t]{2}{*}{1} & . 3 & 0 & & 1 & 1 & 1 & 4 & 2 & 0 & -1 & 1 & 0 & 0 \\
\hline & & & & & & 1 & 2 & 0 & 0 & & & & \\
\hline \multirow[t]{2}{*}{2} & 4 & 0 & & -1 & 2 & -1 & 4 & 1 & 0 & 1 & 5 & 3 & 4 \\
\hline & & & & & & -1 & 1 & 0 & 0 & 1 & 2 & 0 & 0 \\
\hline \multirow[t]{2}{*}{3} & 4 & 0 & & 2 & 3 & -1 & 5 & 2 & 0 & 1 & 6 & 5 & 0 \\
\hline & & & & & & -1 & 1 & 0 & 0 & 1 & 3 & 4 & 0 \\
\hline \multirow[t]{2}{*}{4} & 4 & 0 & & 3 & 4 & -1 & 5 & 2 & 0 & 1 & 6 & 5 & 0 \\
\hline & & & & & & -1 & 3 & 3 & 0 & 1 & 2 & 0 & 0 \\
\hline \multirow[t]{2}{*}{5} & 4 & 0 & & -1 & 5 & -1 & 6 & 3 & 4 & 1 & 7 & 6 & 7 \\
\hline & & & & & & -1 & 1 & 0 & 0 & 1 & 2 & 0 & 0 \\
\hline \multirow[t]{2}{*}{6} & 4 & 0 & $\therefore$ & 3 & 6 & -1 & 7 & 5 & 0 & $2 *$ & 8 & 0 & 0 \\
\hline & & & & & & -1 & 1 & 0 & 0 & 1 & 3 & 7 & 0 \\
\hline \multirow[t]{2}{*}{7} & 4 & 0 & & 2 & 7 & -1 & 7 & 5 & 0 & $2^{\star}$ & 8 & 0 & 0 \\
\hline & & & & & & -1 & 3 & 6 & 0 & 1 & 2 & 0 & 0 \\
\hline
\end{tabular}

* Kill the neutron-beam as it crosses boundary 8 and tally the leakage into leakage tally block 1 . 
The signs for the Sturm's functions at 0 and so are the signs of the following values.

$\begin{array}{cccc}s_{b} & f\left(s_{b}\right) & f^{\prime}\left(s_{b}\right) & f_{1}\left(s_{b}\right) \\ 0 & r & e & \frac{e^{2}}{h}-r \\ \infty & h & h & \frac{e^{2}}{h}-r\end{array}$

Use is made of these equations to reduce the number of times the square root of $e^{2}-h r$ must be determined.

The boundary with the smallest $S_{b}>0$ is identified as the next boundary to be crossed.

\section{Boundary Reflection Routine}

There are two types of reflection presently allowed in the BMC code. They are isotropic and mirror reflection. The isotropic reflection is performed by selecting a set of isotropic random cosines and requiring the signs of the new $\alpha, \beta$ and $\gamma$ to be correct for a reflection back into the region. The equations for a mirror reflection are derived from the vector equation for a mirror reflection. 
TABLE III

EQUATIONS FOR CALCULATING DISTANCE TO BOUNDARY

$I_{\mathrm{d}}$ Equations for distance to boundary

$1 \quad S_{b}=-r / \alpha=-\left(x-x_{0}\right) / \alpha$

$2 \quad S_{b}=-r / B$

$3 \quad \mathrm{~S}_{\mathrm{b}}=-\mathrm{r} / \mathrm{\gamma}$

$4 \quad S_{b}=-r /(\beta-\alpha \cdot a)$

For boundaries $5,6,7$, and 8 the equation becomes

$$
S_{b}=\frac{-e+G_{b} \sqrt{e^{2}-h r}}{h}
$$

where $e$ and $h$ are

5

$$
\begin{aligned}
& e=a x+B y \\
& h=\alpha^{2}+\beta^{2}
\end{aligned}
$$

6

$$
\begin{aligned}
& \mathrm{e}=\alpha\left(\mathrm{x}-\mathrm{x}_{0}\right)+\beta\left(y-y_{0}\right) \\
& \mathrm{h}=\alpha^{2}+\beta^{2}
\end{aligned}
$$

$e=\alpha x+\beta y+\gamma z$

$h=\alpha^{2}+\beta^{2}+\gamma^{2}=1$

8

$$
\begin{aligned}
& \mathrm{e}=\mathrm{A} \alpha\left(\mathrm{x}-\mathrm{x}_{0}\right)+\mathrm{BB}\left(y-y_{0}\right)+\mathrm{C \gamma}_{\gamma}\left(z-z_{0}\right) \\
& \mathrm{h}=A \alpha^{2}+B \beta^{2}+\mathrm{C} \gamma^{2}
\end{aligned}
$$

$$
\vec{n}-\vec{n}^{-}=2\left(\vec{n} \cdot \vec{n}_{b}\right) \vec{n}_{b}
$$

where $\vec{n}=\alpha \hat{i}+\beta \hat{j}+\gamma \hat{k}$ original direction vector.

$$
\vec{n}^{*}=\alpha^{-} \hat{i}+B^{-} \hat{j}+\gamma^{-} \hat{k} \text { final direction vector }
$$

and $\vec{\eta}_{b}=\frac{\partial r}{\partial x} \hat{i}+\frac{\partial r}{\partial y} \hat{j}+\frac{\partial r}{\partial z} \hat{k}$ is the normal vector to the boundary. 
Table IV gives the equation for mirror reflection.

The boundaries can have a reflection albedo associated with them so that the weight and beam strength is modified at the reflection by the albedo for that boundary. The albedos are input as a function of macro energy group.

\section{TABLE IV}

\section{EQUATIONS FOR MIRROR REFLECTION FROM A BOUNDARY}

$I_{d} \quad$ Reflection equations

$1 \quad \alpha^{\prime}=-\alpha, \beta^{\prime}=\beta, \gamma^{\prime}=\gamma$

$2 \quad \alpha^{\prime}=\alpha, \dot{B}^{\prime}=-\beta, \gamma^{\prime}=\gamma$

$3 \quad \alpha^{\prime}=\alpha, \beta^{\prime}=\beta, \gamma^{\prime}=-\gamma$

4

$$
\alpha^{\prime}=\alpha+\frac{2 a(\beta-\alpha a)}{a^{2}+1}, \beta^{\prime}=\beta-\frac{2(\beta-\alpha a)}{a^{2}+1}, \gamma^{-}=\gamma
$$

$$
\alpha^{\prime}=\alpha-2 x \frac{e}{R^{2}}, \beta^{\prime}=\beta-2 y \frac{e}{R^{2}}, \quad \gamma^{-}=\gamma
$$

6

$$
\alpha^{\prime}=\alpha-2\left(x-x_{0}\right) \frac{e}{R^{2}}, \beta^{-}=\beta-2\left(y-y_{0}\right) \frac{e}{R^{2}}, \gamma^{-}=\gamma
$$

$$
\alpha^{\prime}=\alpha-2 x \frac{e}{R^{2}}, \beta^{\prime}=\beta-2 y \frac{e}{R^{2}}, \gamma^{\prime}=\gamma-2 z \frac{e}{R^{2}}
$$

$$
\begin{aligned}
& \ell=A\left(x-x_{0}\right), m=B\left(y-y_{0}\right), n=c\left(z-z_{0}\right) \\
& G=\frac{2[\alpha \ell+8 m+\gamma n]}{\left[\ell^{2}+m^{2}+\gamma^{2}\right]} \\
& \alpha^{\prime}=\alpha-\ell G, B^{-}=\beta-m G, \quad \gamma^{\prime}=\gamma-n G
\end{aligned}
$$


7. The Spectal Square Lattice Geometry Routine

The special square lattice routine is used to locate and calculate distances to boundaries for a neutron-beam in a rectangular array of clad fue 1 rods. The cladding 15 assumed to all be made of the same material and the flux in the cladding is all tallied into the same tally region. The materials and tally regions for the fuels can all be different.

Up to four distances to boundary are calculated at a time thus saving two square root calculations for a neutron going from the moderator in through the cladding, fuel, and out through the cladding.

E. MFPTH - ROUTINE TO OBTAIN THE MATERIAL MEAN FREE PATH

The MFPTH subroutine is used to obtain the material mean free path. The mean free path $(\lambda)$ is equal to the inverse of the total macroscopic cross section and is stored for each material and multi-group.

F. COLLSN - ROUTINE TO PERFORM THE COLLISION MECHANICS

The COLLSN subroutine is used to process the results of a collision. The events which are considered are absorption, fission, and elastic scattering.

1. Neutron Absorption and Fission

The COLLSN routine first reduces the neutron weight to account for absorption. The weight is then tested to see if a scattered neutron is to be produced. The fission probability is checked to see if any fission neutrons were produced. $N$ fission neutrons are produced if $w t . P_{f}+\xi \geq N$ where $\xi$ is a random number and $P_{f}$ is the ratio of the neutron fisston production rate $\left(\nu \Sigma_{f}\right)$ to the total reaction rate $\left(\Sigma_{T}\right)$ in the material. Note that $\mathrm{N}$ will seldom be greater than 1 . The fission neutrons are stored in the fission bank. 
G. FXTLY - ROUTINE TO TALLY FLUX, LEAKAGE AND REACTION RATES

The FXTLY subroutine is used to tally the results of the calculation. The quantities that are tallied are the flux and leakage.

IV. THE BMC CODE: ORGANIZATION AND LAYOUT

The BMC code is comprised of three parts; input routines, the Monte Carlo routines, and the output routines. Each part of the code is maintained in different MAP segments (chain links) and are loaded as needed. The input routines read and process the problem input and the library section tape, preparing the information used by the Monte Carlo and output routines. This information is placed on drum and on a tape which can be used to restart the problem. The Monte Carlo routines are then loaded and executed. After the Monte Carlo calculation is complete, the output routines are loaded to process the Monte Carlo output.

A. DRUM AND TAPE USAGE

The BMC code uses four drum files labeled D1 to D4. The drum word count is used as a means of direct addressing of records. Table $V$ contains the short outline of the usage and contents of the drums during the different parts of the execution phase.

The tapes used by the BMC code are the tape containing the BMC code, the tape containing the multi-group cross section library, and the BMC code restart tape. Only the BMC code restart tape is needed for restarts.

B. RESTART CAPABILITY

The BMC code was designed so that a Monte Carlo calculdtion can be restarted and continued. The way this is done is to save the output from 
the Monte Carlo calculations on a restart tape along with the fission neutrons and the last random number used by the code. The restart tape may also contain the BMC code in absolute or symbolic form, the contents of drums $D 1$ and D2, and the geometry input.

A control word, NWRCD, is used with all but the first two records on the restart tape. NWRCD is normally the number of words in the record. The end of a section, such as the contents of drum DI, is signaled by a record with NWRCD set equal to zero. The end of the restart tape is signaled by a record with NWRCD $=0$ and the second word of the record set equal to -2 . Note that a record written or read with the 1 ist-NWRCD, (NBUF $(N), N=1, N W R C D) \longrightarrow$ will always have two words, even in NWRCD $=0$.

\section{TABLE V}

DRUM USAGE AND CONTENT

Execution Phase

Drum

$$
\text { Input }
$$

Monte Carlo

Output

D1 Cross Sections

Averaged batch cross sections

Cross section information in format and macro fluxes.

for the Monte Carlo routines

D2 Cross section information for Output routines 
The layout of the BMC restart tape is:

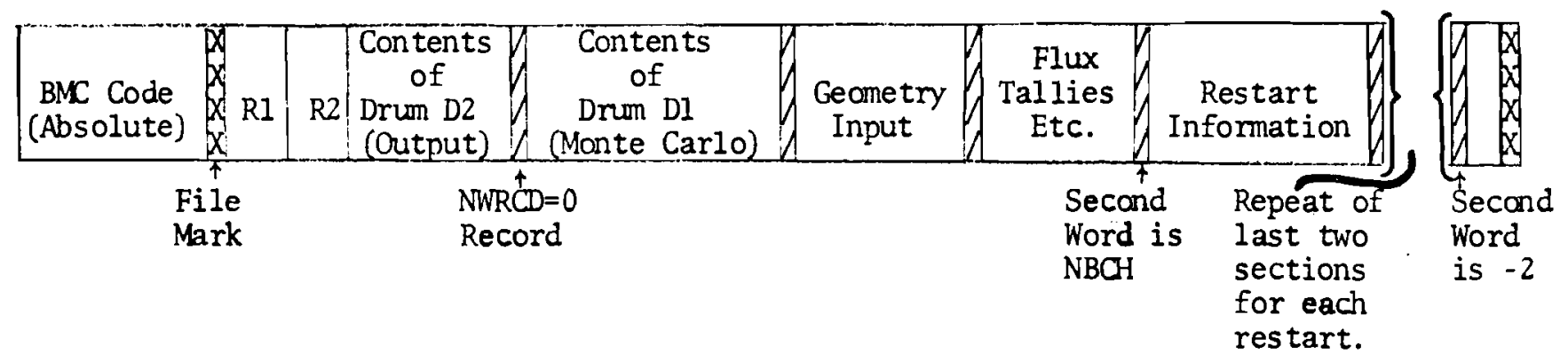

It is not necessary to have the BMC code at the beginning of the restart tape. When the $B M C$ code is given control, it is only required that the restart tape be positioned to read record R1. R1 and R2 are records which contain general information about the restart tape and about the problem. Note that the tallies and restart information are written for each restart. This is a safety factor in case there is an error. If there is a slight error in any of the sections, it is possible to redo the input phase of the BMC code and still restart using the old restart information and saving the old flux tallies.

\section{PROGRAM TIMING}

The amount of time in seconds spent in various portions of the code is clocked and printed. The times which are written are:

- The compilation and load time.

- The time required in the input routines.

- The time required to process each batch.

- The total time for the Monte Carlo calculation.

- The time required to process the output

The total time is also printed along with each of the above times. 
The clock routines are also used to check the amount of time used for a batch. If there is not enough time left to do another batch and allow 1 minute to finish the output then the Monte Carlo is terminated and the output routines are called.

\section{PROBLEM SIZE}

The BMC code was designed to be flexible as to the size of problem which can be run in terms of energy, cross section, and geometry detail. The BMC code has been used for problems which contained as little as one material in one region and for problems which contained as many as 6 materials, 43 energy groups, and 135 geometry and tally regions.

The dimensions of the storage arrays are entered using PARAMETER cards and most of the storage arrays are maintained in a data element which is placed in the required subroutines using an INCLUDE statement. This makes it easy to change the dimensions for a problem.

The dimensions which are built into the standard version allow for 50 energy multi-groups with 6 materials, 20 tally regions, 2 leakage tally set, 60 geometric regions, 60 boundaries, and 1000 fission particles in the fission bank. As long as the array does not exceed a set size determined by the computer core area the number of materials and groups are variable. This array size is calculated simply as:

$$
I G P * I H P * N * \text { NMAT }
$$

where: $\quad$ IGP $=$ the number of groups

IHP = the length of the cross-section set generally IGP +3

$\mathrm{N}$ = the Legendre expansion plus 1. (Example: for $\mathrm{P}_{3} \mathrm{~N}=4$ )

NMAT $=$ The number of materials used in the calculation 
The dimensioning parameters for the BMC code are defined in Appendix I.

\section{THE BMC OUTPUT ROUTINES}

The BMC output routines prepare the output and print it. The two main tasks in preparing the output are preparing averaged values of fluxes, cross sections, reaction rates, and leakages along with some other cell parameters and obtaining statistics for the values which are calculated.

\section{A. STATISTICS}

Statistics are obtained by processing a series of equal sized batches and averaging the results for the batches. It is possible to leave some of the first batches out of the average. This might be done to damp out the effects of the source distribution. There are two common types of average values obtained from the Monte Carlo calculation. One type is a direct answer such as flux or reaction rate and the other type is a ratio such as flux averaged cross sections. The statistical error for a single averaged value $\bar{a}$ is $S_{\bar{a}}$ where $S_{\bar{a}}$ is the error for one standard deviation. Let $N$ be the number of equal sized batches and $a_{n}$ be the value of a for the $n^{\text {th }}$ batch. The values of $\bar{a}$ and $S_{\bar{a}}$ then become:

$$
\bar{a}=\frac{1}{N} \sum_{n=1}^{N} a_{n}
$$

and

$$
\begin{aligned}
S_{-} & =\left[\left\{\sum_{n=1}^{N} a_{n}^{2}-N(\bar{a})^{2}\right\} / N(N-1)\right] 1 / 2 \\
& =\left[\left\{\sum_{n=1}^{N} a_{n}^{2}-\left(\sum_{n=1}^{N} a_{n}\right)^{2} N\right\} / N(N-1)\right] 1 / 2
\end{aligned}
$$


The statistical error for a ratio $R$ is

$$
S_{R}=\left[\sum_{n=1}^{N} a_{n}^{2}-2 R \sum_{n=1}^{N} a_{n} b_{n}+R^{2} \sum_{n=1}^{N} b_{n}^{2}\right]^{1 / 2} / \sum_{n=1}^{N} b_{n},
$$

where

$$
R=\sum_{n=1}^{N} a_{n} / \sum_{n=1}^{N} b_{n}
$$

Note that for a ratio the values of $a$ and of $b$ may have large statistical errors and the statistical error of the ratio can be very small or even zero. This is accounted for by the correlation term $\left(-2 R \sum_{n=1}^{N} a_{n} b_{n}\right)$.

The statistical error is written out in a way which reduces the printing space. The statistical error is forced to have the same exponent as the value. If a value and $i$ ts error were $1.0685 \times 10^{4} \pm 5.2 \times 10^{2}$ then the numbers would be printed out as $1.0685+040.052$.

\section{BROAD ENERGY AVERAGING GROUPS}

The output routines make use of one more type of energy group also called a broad group. These broad group energy boundaries must coincide with the multi-group boundaries. The broad groups do not need to cover the entire energy range or to have joining boundaries. In fact, the boundaries can overlap. To illustrate, suppose there were five multi-groups; then the four broad groups could contain groups $1-4,1-3,1-2$ and 4 respectively. Note that group 5 is never included, group 4 is by itself, and the groups 1-4 are overlapping.

The broad groups are used for flux and reaction rate integrals. The broad group fluxes are also used to obtain statistics on the ratios between different 
energy broad groups or between regions for a given broad group.

\section{c. PRINTED OUTPUT}

The output code prints out the results of the Monte Carlo calculation. First the averaged code check tallies are printed out. Next the multi-group and the broad group fluxes and leakages are printed. The multi-group average cross sections and the broad group reaction rates for each isotope in each region are printed along with the reaction rates for the region. The reaction rates for the entire cell along with an infinite and effective multiplication constant follow. Finally the multi-group fluxes for each region are printed and/or plotted.

D. THE DEFINITION OF FLUX

The flux as calculated by the Monte Carlo routines is really the fluxenergy volume integral, which has the units of centimeters per second. This flux integral is what is used with cross sections to obtain reaction rates and is usually more useful than true flux.

The flux is printed out in the following three forms by the output routines:

1) Flux-volume-energy integral - Flux-cm/xec.

2) Flux-energy integral $\mathrm{Flux} / \mathrm{cm}^{2} / \mathrm{sec}$.

3) Flux $\mathrm{Flux} / \mathrm{eV} / \mathrm{cm}^{2} / \mathrm{sec}$.

\section{E. PLOTTING THE MULTI-GROUP FLUXES}

The multi-group region and/or region averaged fluxes may be plotted. The plotting can either be a rough plot on the printed output or a neat plot using the Calcomp plotter. If the printer is used, only the flux 
points are plotted. If the Calcomp plotter is used, the flux points along with their error bars can be plotted.

The plots of the fluxes serve mostly as visual checks against gross errors or to observe how the flux spectrum changes from region to region. The fluxes can be normalized over part or all of the energy range and plotted on the same curve so that changes in spectral shape are readily visible.

F. SPECIAL OUTPUT

There may be special values which are desired which are not part of the regular output. These special values can often be obtained by simple changes to the BMC output routines. For some problems it might be better to rewrite the output routines.

\section{THE BMC-MG INPUT ROUTINES}

The BMC input routines are used to prepare the data needed by the BMC Monte Carlo and output routines. They also prepare much of the restart tape. The input routines receive information from two sources; card input and the cross-section tape. The input routines also print the input information.

The card input is read by two different types of formats. The first type uses standard FORTRAN formats. The second type, used mainly for the geometry input, requires a special card reading routine. 
A. SPECIAL CARD INPUT ROUTINE, CRDRD

The CRDRD* routines read cards of two format types. They are called $P$ or $U$. The $P$ or $U$ is read in column $I$ on the first card of a section of input called a record. The record is ended with an end of record marker, R. Cards with an asterisk in the first column can be used before the $P$ or $U$ type card as comment cards.

The $P$ card indicates that the data will be integers using 3 column fields (13). As many as 24 or as few as one data word can be on one card. Each field of three columns is checked. If a slash, /, is in any one of the three columns, that field along with the rest of the card is skipped. The record is ended with a $R$ card.

The $U$ card indicates that the input will be a mixture of integer and floating point numbers. A number is considered to be floating point if it contains a decimal point and/or an exponent. Otherwise it is considered to be an integer. The data word must be contained in a field of not more than 12 columns. The data words do not have to be right adjusted. Fields of less than 12 columns can be used by placing a comma after the data word. The next field would then start right after the comma. Note, the routine assumes that the maximum field length is 12 columns so if a number uses more than 12 columns, it will be split into two numbers. If the columns are left blank, a zero will be entered.

Again, as with the $P$ card, a slash in any column of a field will cause that field and the rest of the card to be skipped. The space after the slash may be used for comments as it is not examined.

*Written and coded by Kent B. Stewart, Battelle-Northwest Laboratory. 
Several identical words can be entered simply by putting the number of words followed by an asterisk. For example, 11*4.0 would enter the number 4.0 into 11 consecutive words of the record. The field of 12 columns starts right after the * when the * is used.

B. DESCRIPTION OF THE BMC-MG CODE PROBLEM INPUT

The problem input numbers for each input card will be described along with their names and format. It will also be indicated when the input card is needed.

The first two input cards are always needed. The first card contains a $B C D$ title which is printed at the top of each page of the output along with the date and page number. The second card contains execution control information. Card 3 and the input to the source routine are always required if a Monte Carlo calculation is done. The input for the output routines is always required if the output calculations are done. The energy-material-geometry description input is not needed for a normal restart.

The restart tape is called IMCT when it is being written. It is called INC $\theta$ when it is being read. IMC $\theta$ will always be on logical tape unit $A$ while IMCT can be on unit $A$ or $B$.

C. CROSS-SECTION INPUT

The cross-sections available with the code are in the same format as ANISN uses. The following description is taken directly from the ANISN USER document. (Reference 5 of the main document)

ANISN expects a table of cross sections for each group, $g$, of each material in the following format: 
Position

1

-

IH $\dot{T}-2$

IHT - 1

IHT

$\mathrm{IHT}+1$

IHS-I

IHS

IHS+1

$\dot{\cdot}$

\section{Cross Section Type}

activity

"

"

absorption

nu $x$ fission

total

$\left.\begin{array}{c}\sigma_{g+N U S \rightarrow g} \\ \vdots \\ \sigma_{g+l \rightarrow g}\end{array}\right\}$ upscatter 5

$\sigma_{g \rightarrow g}$

$\left.\begin{array}{c}\sigma_{g-l \rightarrow g} \\ \cdot \\ \sigma_{g-N D S} \rightarrow g\end{array}\right\}$ downscatter 5

Thus the parameters IHT, IHS, and IHM completely describe the format of the cross sections. If there are no activity cross sections, IHT $=3$. If there is no upscatter IHS $=$ IHT +1 . If there is no downscatter IHM $=$ IHS (i.e. a one group problem). If there is upscatter ANISN will compute a total upscatter cross section for each group of each material and place that cross section in position $I H M+1$. The activity cross sections are used only for activities $(22 \$, 23 \$)$.

\footnotetext{
${ }^{5}$ US is the number of groups of upscatter. NDS is the number of groups of downscatter.
} 
The $P_{L}$ cross section tables must correspond in format to the $P_{0}$ tables eyen though the transfer coefficients are the only numbers used. Note that the $P_{L}$ cross sections must contain a $(2 L+1)$ term. Some $S_{n}$ codes supplied this term internally. (e.g. DTF-11 multiplied the $P_{1}$ cross sections by $3.0)$.

\section{FIDO FORMAT}

Several arrays including the cross-sections are input in FIDO format. FIDO is a data reading subroutine which we have obtained from the ANISN code. The following description of this input is taken from reference 6 of the main body of the report.

All numerical data is written in the same format. Each data card consists of six twelve-column data fields. Each data field consists of three subfields of two, one, and nine columns respectively. The contents of the first two subfields define the operation to be performed on the data contained in the third subfield. The second subfield may contain either a blank or one of the following characters: $\$, \star, R, I, T, S, F, A,+,-$. The first subfield may contain either a blank or an integer from 1 to 99.

$\$$ indicates the beginning of an integer array. The first subfield identifies the array. 3rd subfield is blank.

* indicates the beginning of a floating point array. The first subfield identifies the array. 3rd subfield is blank.

$R$ indicates that the data contained in the third subfield is to be entered several times in succession. The first subfield defines the number of successive entries or Repeats. 
I indicates linear Interpolation between the data in the associated third subfield and the following third subfield. The first subfield defines the number of interpolations between the two data entries.

$T$ indicates Iermination of data reading. All following data and data in the associated first and third subfields is ignored.

$S$ indicates skip. The first subfield defines the number of entires to be skipped. The third subfield may contain the first entry following the skips.

$F$ indicates that the remainder of the present array is to be Filled with the data entry in the third subfield. Any entry in the first subfield is ignored.

A indicates Address modification. The next non-blank data entry is entered in the Nth location of the present array where $N$ is an integer entry in the third subfield associated with the A. Any entry in the first subfield is ignored.

+ or - indicates exponentiation. The data entry in the third subfield is multiplied by $10^{ \pm N}$ where $N$ is the entry in the first subfield. This option allows more significant digits if necessary.

Integer data in the third subfield must be right justified. Floating point data may be written with or without an exponent and with or without a decimal point. If the decimal point is not included, it is assumed to be immediately to the left of the exponent field. If there is no exponent, the decimal point is assumed to be at the extreme right of the nine column subfield. 
The following restrictions must be observed when writing data for ANISN:

1) Floating point zeroes must be written as 0 . or 0.0 . A .0 is read as a blank. (-zero)

2) Blanks are ignored.

3) If an I is specified in any data field, the next data field may not be either blank or an A entry.

4) The third subfield of a data field containing a \$ or a * may contain any integer, $N$. The next data entry is assumed to be the $(\mathrm{N}+1)$ th member of the array. Normally this third subfield is blank and is interpreted as zero. 
VII. DESCRIPTION OF CARD INPUT TO THE BMC-MG CODE

Card 1 Format (12A6) BCD title card

Card 2 Format (11 I5, 5X, 2A6, 7X I1) program contro1

NPRBTP

Identification number of the Problem tape. If this

is a restart, the number must match the number on the restart tape.

LSTRT

0, Initial start. IMCT $=B$.

1, Restart, no changes, tape to be written to IMCT $=A$.

2, Restart but do input calculations over.

3 , Restart but do the geometry input calculations over.

(For LSTRT $=2$ or 3, IMCO $=A$, IMCT $=B$ )

IMCTSV

0 , Write and save the restart tape

66 , Do not write a restart tape

LCONT

0 or 1, Do a Monte Carlo calculation

2 , Go to the output routines after the input. Do

not do a Monte Carlo Calculation

LSTOP

0 , Do complete problem.

1, Do input calculations only.

2, Do input and Monte Carlo calculations but not the output processing.

LCOPY

If 1, copy the restart tape IMCO to IMCT and use IMCT.

IMCO $=A$, IMCT $=$ B. $\quad($ Active only if LSTRT $=1$.

LSKNS

LSKNS is used when LSTRT > 0 .

0 , Position restart tape to the normal (last) restart.

If LSKNS > 0, then LSKSV must equal 77 or calculation will not be made. 
$\begin{array}{ll}\text { LSKNS (cont) } & 1, \text { Start the tallies anew. } \\ & 2, \text { Use the restart dated (DLSKA, DLSKB). } \\ & \text { Safety factor for use of LSKNS }>0 \text {. Must equal } 77 \\ \text { LSKSV } & \text { when LSKNS }>0 . \\ & \text { Number of recoverable errors before termination of } \\ \text { NERCV } & \text { program execution. } \\ & 0, \text { Use previous set of importance and roulette } \\ \text { IMPRD } & \text { parameters for restart. } \\ & 55, \text { Read a new set of importance and roulette } \\ & \text { parameters with normal restart. }\end{array}$

Card 3 Format (215, E10.0, 2I5, 5X, 015) Monte Carlo batch control. (Card 3 not needed if LCONT $=2$ ).

NBTCH The number of batches for this case. (Not total number of batches for the calculation.

NPTPB The number of particles (source or fission) to follow in each batch.

The next three input numbers are used only if the thermal tally option is used (see Card 4, LTALY $=2$ ).

RTHRT

The ratio of the weight leaving to that entering the thermal tally range.

JTHRT The energy group to which particles must be slowed down before they are used in (enter) the thermal tally range.

JTHRTX The maximum energy group a particle may achieve in the thermal tally range. 
RND

RND can be used to change the initial random number. If RND is left blank, the octal number 343277244615 or the random number at the end of the last batch will be used. Otherwise, the octal number RND read in will be used.

Card 4 Format (14I5) Problem description parameters. (Card 4 not needed if LSTRT $=1)$.

\begin{tabular}{|c|c|}
\hline LPRB & $\begin{array}{l}\text { Problem type control. } \\
0 \text {, Fission descendant problem. } \\
\text { 1, Direct source problem. } \\
2 \text {, Direct source plus fission descendant. } \\
\text { If LPRB is less then } 0 \text { the fission spectrum must be } \\
\text { input from cards (card } 7 \text { ), otherwise both neutron } \\
\text { and/or gamma fission spectra built into code is used } \\
\text { depending on problem type (i.e. neutron or neutron- } \\
\text { gamma coupled) }\end{array}$ \\
\hline JMAXX & Total number of energy groups. \\
\hline JGAMX & Number of gamma energy groups. \\
\hline MMAX & Number of materials. (Not including voids.) \\
\hline NBNDX & $\begin{array}{l}\text { Number of boundaries used in the geometrical } \\
\text { description. }\end{array}$ \\
\hline NHMAX & Number of geometrical regions. \\
\hline NSPRG & Number of "Special" geometrical regions. \\
\hline NFMAX & Number of tally regions. \\
\hline NLKTLY & Number of leakage tally sets. \\
\hline
\end{tabular}


LTALY

0 or 1; Use only one tally set for each tally region.

2

2; use two tally sets (make special thermal tallies) for each tally region.

NOMGX

Number of boundary reflection albedo sets. Read in with CRDRD Record 3.

Card 5 Format (1415) Cross-section input control

NICRD

NITAP

IHTX

IHSX

IHMX

NANRX

LANRX

LDKRN

NCXPR
Number of isotope cross-section sets read from cards. Number of isotope cross-section sets read from tape. Position of total cross-section.

Position of self-scatter cross-section term.

Length of cross-section table.

Order of scattering $n$ where $P_{n}$ is the highest order of scatter

Type of scattering technique to be used

0 or 1 - polynomial rejection technique

2 - polynomial rejection technique with point detector

3 - discrete angle technique

4 - discrete angle technique with point detector

Diffusion kernel control (presently not available).

0 - Use normal transport only

1 - Use diffusion kernel in specified groups.

Cross-section print option

1 - print cross-section

2 - no cross-sections printed

-1 - print angular and transfer data 
INPUN

NDTC

LRAD
Print options for discrete angle technique

0 - no printing

1 - errors only

2 - also moments

3 - also information from subroutine GETMUS

Number of point detectors (if negative the units

are in inches)

Number of conversion tables for region averaged

activities (this includes flux to dose conversion

factor tables). A gamma and neutron flux to dose

conversion table is built into the code and con-

trolled by card $0-5$ (page 65). If LRAD is less

than 0 , the conversion tables are input by card

set $\theta-4$.

Card 6 Format (6E12.0)

EJG(JMAXX)

The upper energy limit for each group starting with

the highest energy neutron group. (JMAXX +1

entries needed.)

Card 7 Format $(I 2, A 1, E 9.0)$ Needed if LPRB $<0$ as input. This is FIDO Format described on page 39.
FSPEC (JMAXX)
A $1^{*}$ in columns 2 and 3 must be on the first card followed by the fission spectrum starting with the highest energy neutron group. A $T$ in the third column of a 12 column field must end the array. 


\section{CROSS SECTION INPUT}

Material Description and Isotopic Mix Instructions

Repeat Card 8 and accompanying Card 9's for each material

Card 8 Format $(15,5 X, 3 A 6)$

NISO

Number of isotopes in this material

$\operatorname{BCDM}(3)$

Alpha numeric information describing material

Card Set 9 Format (I5, E15.0) Need NISO Card 9's for each Card 8

ISOM

Identification number for isotope

CONM Density of isotope in material of Card 8.

Cross Sections

Card A Format (AI, I4, 412, I3, I1, 9A6) Cross section set description

$\$$

MATID

IFRT

NLMBI

IHT I
Dollar sign

Isotope ID Number

1. DTF IV format cross sections

$(2 \ell+1$ factor must not be included in legendre expansions)

2. ANISN format cross sections

$(2 \ell+1$ factors must be included in legendre expansions)

3. Multiplies ANISN format cross-sections by $2 \imath+1$ factor.

Order of scatter $n$ where $P_{n}$ is the highest order of scatter.

Position of tutal cross section. 
IHSI

Position of self-scatter cross section

IHMI

Table length of cross section set

IFSPI

Do not use this.

HOLN

Alpha-neumeric information describing isotope.

Card B ANISN Format-14* in the first three columns, rest of card blank

DTF IV Format - Title card (16A4)

Card set $\mathrm{C}$

Cross sections in format defined by IFRT of Card $A$

Card D

ANISN Format - "T" in column three - rest of card blank

(follows highest order of scatter cross sections for each isotope just before next card A).

DTF IV Format -- Leave this card out.

The next section of records contains the geometry and region data. A set of data which is read by the CRDRD routines will be designated as CRDRD Record. This section is not needed if LSTRT $=1$.

CRDRD Record 1, Format $U$ (Mixed floating point and integer)

Boundary description record

The following information is repeated for each of the NBNDX boundaries. IDB

The index telling the boundary type.

If the first value of IDB is negative, all boundary

dimensions are in inches, otherwise they are in centimeters. 
Boundary description record (continued)

IEM
Albedo index. Gives type of reflection
0 , no reflection
1, mirror reflection using albedo set 1 .
2, mirror reflection using albedo set 2 .
3. mirror reflection, unit albedo.
4. isotropic reflection, unit albedo.

Boundary Parameters

The boundary parameters depend on the boundary type. The parameters and their order are:

IDB

1

2

3

4

5

6

7

8
Parameters

Xo

Yo

Zo

b, a

R

$R, X_{0}, Y_{O}$

R

$K, X_{O}, Y_{O}, Z_{O}$,

$A, B, C$
Dimensions in centimeters or inches, depending on IDB.

Plane $x=x_{0}$.

Plant $y=y_{0}$.

Plane $z=z_{0}$

Plane $y=a x+b$.

$R=$ radius of cylinder centered at origin.

Cylinder, centered at $X_{0}, Y_{0}$.

sphere, centered at origin.

General boundary 
For example, a record for 5 boundaries could be

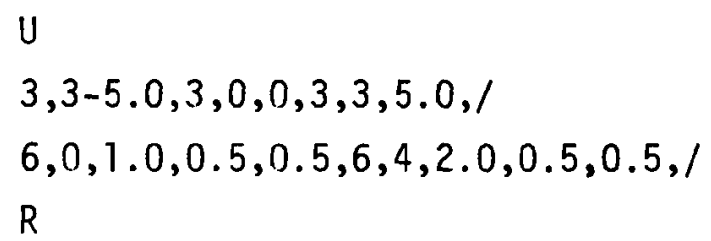

CRDRD Record 2, Format P (24I3)

Region description record.

This record contains four words for each region plus four words for each boundary in each region.

NBH Number of boundaries needed to describe this region LSPH 0 , this is a normal region. $L$, the number of the "special" region to use in this region. ( $(\leq N S P R G)$

MATH Material to use in this region. If $M A T H=0$, the region is an external void. A neutron in an external void is rejected. If $M A T H=-1$, the region is an internal void. The neutron-beam will cross the region without collisions or alteration.

IFH The tally region number for this region. If IFH $=0$, no tallies will be made for a neutron -beam in this region. Note that the flux in an internal void can be tallied by setting IFH $\neq 0$.

The following four parameters are entered for each of the NBH boundaries of the region. 
IGDH

$G$ is the sign of the boundary and

$D$ is the leakage tally set index.

$G=\operatorname{sign}$ of $I G D H$

$D=A B S(I G D H)-1$

If $D=0$, f.e., IGDH $= \pm 1$, no leakage is tallied.

If $1 \leq D \leq 50$, i.e., IGDH $= \pm 2$ to \pm 51 , the leakage will

be tallied into the leakage tally set $D$ and the

neutron-beam will be killed.

If $D \geq 51$, then the leakage across the boundary will

be tallied into leakage tally set D-50 but the neutronbeam will not be killed.

IBHH

Boundary index.

IOIH

The region the neutron will most likely enter next

if it leaves the region across this boundary.

IQ2H

The region to start the region search in if region

IQIH fails. (Usually this will be the lowest

numbered region which the neutron could enter.)

IGDH to IQ2H are repeated for all of the boundaries of the region and then the data is repeated starting with NBH for the next region.

Cards 10 to 14 not needed if NSPRG $=0$

Card 10 Format (14I5)

LTSPS(NSPRG)

LTSPS is the type of special region. Enter a value for each of the NSPRG special regions. Currently the only type of spectal region available is the square lattice of clad fuel rods $($ LTSPS $=1)$. 
Cards 11 to 14 contain input for the special square lattice geometry routine. If a different special geometry routine were being used, the input would be different but the input cards would be in the same position.

Cards 11 to 14 are repeated for each special region.

Card 11 Format (14I5)

NRX

NRY

MATCL

IFTCL

MATFL

IFTFL

$\mathrm{XC}$

YC

DXC

DYC

RDF

$\mathrm{RDC}$
Number of rows of rods in the $x$ direction.

Number of rows of rods in the $Y$ direction.

Material number of the cladding.

Tally region of cladding.

Material number of the fuel in the rods. If

MATFL $=0$, the material numbers for each rod will

be read using Card 13-

Tally region of the fuel rods. If IFTFL $=0$, the

tally region numbers will be read using Card 14.

Card 12 Format (7E10.0) (A11 dimensions in centimeters or inches, depending on IDB.)

\begin{tabular}{|c|c|}
\hline$X C$ & $\begin{array}{l}X \text { coordinate of the center of the lower left hand } \\
\text { corner rod, rod }(1,1) \text {. }\end{array}$ \\
\hline$Y C$ & $\begin{array}{l}Y \text { coordinate of the center of the lower left hand } \\
\text { corner rod. }\end{array}$ \\
\hline $\mathrm{DXC}$ & Lattice spacing in the $x$ direction. \\
\hline DYC & Lattice spacing in the $Y$ direction. \\
\hline $\mathrm{F}$ & Radius of the fuel rod. \\
\hline & Radius of outer edge of cladding. \\
\hline
\end{tabular}


Card 13 Format (1415) Needed only if MATFL $=0$.

$\operatorname{MATXYS(NRX,J)\quad The~material~numbers~of~all~the~rods~in~the~} J$ 'th

row.

Repeat card 13 for $\mathrm{J}=1,2-\cdots, \mathrm{NRY}$.

Card 14 Format (14I5) Needed only if IFTFL $=0$.

IFTXYS(NRX,J) The tally region number of all the rods in the $J$ 'th

row.

Repeat card 14 for $\mathrm{J}=1,2, \ldots$, NRY.

CRDRD Record 3 Format $U$ (Not needed if NOMGX $=0$ ).

OMGAB (JMAXX, NOMGX)
The boundary reflection albedos. Enter a value for each macro-energy group for the first albedo set. Then repeat for the next set until NOMGX sets have been entered.

Card 15 Format (215) Energy and zone weighting control.

NERGX Number of energy weighting zones

NWTZX Number of geometrical weighting zones

CRDRD Record 4 Format U Russian Roulette and importance weight data IXWZ (NHMAX) Weighting zone for each geometrical region (not needed if NWTZX $=1$ ).

IVW(NERGX) The upper group for each energy weighting range $(I V W=1$ if NERGX $=1)$. The ranges must be continuous, i.e., the upper energy group of one range except the first is the lower energy group of the range above it. 
Repeat the following six parameters for each energy weiahting zone and geometrical weighting zone. The WTX parameters are read for each geometrical weighting zone, then the WTXH etc. for each energy weighting zone until NERGX energy weighting zones are completed.

$\begin{array}{ll}\text { WTX (NWTZX) } & \text { Minimum neutron weight before Russian Roulette is } \\ \text { played. } & \text { Neutron survival weight in each geometrical zone. } \\ \text { WTXH(NWTZX) } & \text { Maximum weight of particle before splitting in } \\ \text { WTMH(NWTZX) } & \text { each geometrical zone. } \\ \text { BEMNH(NWTZX) } & \text { Minimum beam strength before Russian Roulette is } \\ \text { played in each geometrical zone. } \\ \text { BEMXH(NWTZX) } & \text { Beam survival strength in each geometrical zone. } \\ \text { ZWTH(NWTZX) } & \text { Importance weight for each geometrical zone. }\end{array}$

Card set 16 Format (4I5, 3E10.0) Point detector description

Repeat card 16 NDTC times (Card 5)

LEDT

Type of detector; only type 1 allowed at this time

If LEDT is negative the dimensions are in

inches, otherwise centimeters.

IHDT

Region the point detector is in.

IFDT

Tally region for point detector.

MIDT

Material number of point detector (used only to give

reaction rates at the detector)

-1 if void

XDT

$X$ coordinate point detector location

YDT

$Y$ coordinate point detector location

ZDT

$Z$ coordinate point detector location 
Source Routine Input

The source routine input is needod whenever a Monte Carlo calculation is made. It is not put on the restart tape. The input described here is for the $B M C-M G$ general source routine. If a different source routine is used, the input would go here.

Card S-0 Format (3I5) Needed for any Monte Carlo Calculation

NREG

The number of source regions.

Cards S-1 through S-6 are repeated NREG times

INCH

0 - dimensions in centimeters

-1 - dimensions in inches

NEGM

The number of source energy spectra

Cards S-7 through S-9 are repeated NEGM times

after all sets of cards S-1 through S-6.

Card S-1 Format (4I2, 2X, 2E10.0) LRGGS Type of geometrical coordinate system to use in this source region.

1, Rectangular

2, Cylindrical

3, Spherical

4, General Geometry

NEGS

1 , not used; must equal 1 .

LANGS

Angular biasing option for source

o or 1 - Isotopic

2 - Mono-directional beam

Supply the direction cosines on Card S-6 
3 - Isotropic on X half-plane depending on sign of ALS

4 - Isotropic on $Y$ half-plane depending on sign of BTS

5 - Isotropic on $Z$ half-plane depending on sign of GMS.

ALS, BTS, and GMS are input on Card S-6.

6 - Particles selected only in the solid angle defined by direction cosines UMIS $\leq \mu \leq$ uM2S (Card S-6)

7 - Particles selected from distribution $P=1 /(1-Q U M S * U)$ with biasing

8 - Particles selected from distribution $P=1+$ QUMS*U without biasing (an anisotropic distribution)

9 - Particles selected from distribution $P=1 / 2\left(1+Q U M S^{*} U\right)$ with biasing QUMS is set on Card S-6

IQRGS The geometrical region to look in first to locate a neutron born in this source region. If the neutron is not in region IQRGS or if IQRGS $=0$, a region search will start with region IWRGS +1 .

PRBRS The probability that a neutron is born in this source region relative to the other regions. PRBRS does not have to be normalized. 
WTPRS

Weight of a neutron born in this source region.

1.0. If WTPRS $=0$, it will be set to 1.0. )

Card S-2 Format $(4 \mathrm{I} 2,2 \mathrm{X}, 6 \mathrm{E} 10.0)$ Needed on 1 y for rectangular geometry, LRGGS $=1$.

LXD

Type of spacial distribution in the $X$ direction.

1, Point at $x_{1}$

2, Uniform between $x_{1}$ and $x_{2}$.

3 , Cosine $\frac{\pi}{2} \cdot \frac{\left(x-X_{0}\right)}{H X}$ between $x_{1}$ and $x_{2}$.

LYD

Spatial distribution in the $Y$ direction. (Same

distributions as under LXD.)

LZD

Spatial distribution for the $Z$ direction.

LHOL

The number of cylindrical holes to include in this

source region. (It is often necessary to exclude a

cylindrical region such as a fuel rod from a rectan-

gular source region. This may be done by using "holes",

which are infinite cylinders in the $Z$ direction. The

hole parameters are input on Card S-2B.

$\times 1$

Spatial coordinate 1 imits

Y1

Z1

$\times 2$

$Y 2$

22 
Card S-2A Format (6E 70.0 ) Need if LXD, LYD, or LZD $=3$.

$x Z$

The values for $x_{0}$ and $H_{x}$ used in the cosine distribution

HX

$(L X D=3)$. Same for $Y$ and $Z$.

YZ

HY

$\mathrm{ZZ}$

$\mathrm{HZ}$

Card S-2B Format (6E10.0) Needed only if LHOL $>0$.

XHOL

The hole is a cylinder centered at (XHOL, YHOL)

with $X$ radius RDA. Repeat as many cards as necessary, two sets of parameters per card.

YHOL

ROA

Card S-3 Format $(5 \mathrm{I} 2,6 \mathrm{E} 10.0)$ Needed only for cylindrical geometry, (LRGGS = 2).

LIB

$0, Z$ cylinder

1 , X cylinder

2, Y cylinder

LRD

The type of spatial distribution in the radial

direction.

1 , fixed radius, $r=r$.

2, uniform in volume between $r_{1}$ and $r_{2}$.

$3, J_{0}\left(2.405 * r / r_{c}\right)$ between $r_{1}$ and $r_{2}$.

The radius is centered at $\left(X_{0}, Y_{0}\right),\left(Y_{0}, Z_{0}\right)$, or $\left(x_{0}, z_{0}\right)$ 
LZD

The type of spatial distribution in the axial

direction.

1, plane at $z=z_{1}, x=x_{1}, y=y_{1}$

2, uniform between $z_{1}$ and $z_{2}, x_{1}$ and $x_{2}$, or $y_{1}$ and $y_{2}$.

3 , cosine $\frac{\pi}{2} \frac{\left(z-z_{0}\right)}{H_{2}}$ between $z_{1}$ and $z_{2}, x_{1}$ and $x_{2}$, or $y_{1}$ and $y_{2}$.

IXPM

IXPM and IYPM tel1 which quadrants the source neutrons may fall in. If IXPM $=0$, then $X$ can be plus or minus.

IYPM If $\operatorname{IXPM}=+1$, then $X$ can only be plus. If IXPM $=$ -1 , then $X$ can only be negative. IYPM controls $Y$ in the same way.

$x Z$

$Y Z$ Center line of the cylinder.

RI Spatial coordinate limits

$\mathrm{R} 2$

21

Z2

Card S-3A Format (3E10.0) Needed if LRD or $L Z D=3$.

$\mathrm{RC}$ Extrapolation radius, $r_{c}$.

ZZ

$\mathrm{Z}_{0}$

$\mathrm{HZ}$

$\mathrm{H}_{\mathbf{Z}}$

Card S-4 Format (4I2, 2X, 6E10.0) Needed for spherical geometry, LRGGS - 3. LRD Type of spatial distribution

1 , fixed radius, $r-r_{1}$. 
2, uniform in volume between $r_{1}$ and $r_{2}$.

3 , $(\sin U) / U$ where $U=\pi r / r$. Between $r_{1}$ and $r_{2}$.

The radius is centered at $X_{0}, Y_{0}, Z_{0}$.

IXPM Quadrant definition. See IXPM under Card S-3.

IYPM

IZPM

$X Z$

Coordinate definitions: Center of the sphere relative to the origin used to describe the geometrical boundaries in CRDRD record 1 on page 46 .

YZ

22

R1

R2

RC

Ird S-5 Format $(4 \mathrm{I} 2,2 \mathrm{X}, 6 \mathrm{E} 10.0)$ Needed for general geometry, LRGGS - 4. LRD Type of spatial distribution

2, Uniform in volume between two surfaces (Tested for two ellipsoids; one wholly inside the other)

IXPM Quadrant definition. See IXMP

IYPM Under card S-3

IZPM

$X Z$

$\mathrm{YZ}$

Coordinate definitions as above

$\mathrm{ZZ}$ 
AG1

Parameters in the equation $A G * x^{2}+B G^{*} y^{2}+C G * Z^{2}=$ $\mathrm{RG}^{2}$

$B G 1$

CGI (The number 2 equation represents the outer surface)

Card S-5A Format (7E10.0)

$A C 2$

$B G 2$

CG2

RG1

RG2

Card S-5 Format (6E10.0) Direction angles and biasing factor, refer to card S-1. (Needed if LANGS greater than 1.)

ALS

BTS

GMS

Direction cosines of the solid angle

UMIS

UM2S

Cosines of the solid angle limits

QUMS

Biasing factor

Card S-7 Format (3I2, 4X, 6E.10.0) Source strength and spectra control. LETS Type of source

0 , Enter spectrum by cards

1, Pu metal (spontaneous fission)

2, $\mathrm{PuO}_{2}$ (spontaneous fission and alpha, neutron)

$3, \mathrm{PuF}_{4}$ (spontaneous fission and alphd, neutron)

4, PuBe (spontaneous fission and alpha, neutron)

5, PuC (spontaneous fission and alpha, neutron)

6, $\mathrm{Pu}_{n}\left(\mathrm{NO}_{3}\right)_{n}$ (spontaneous fission and alpha, neutron) 
NGAZ

Type of calculation

1, Neutron only

2, Gamma only

3, Coupled neutron gamma

KWSP

Spectrum weighting

0 - Read from cards. Card S-9.

1 - Internally set equal to 1.0 for each group.

-1 - Inverse of the spectrum.

For LETS equal 0 must read weights by group.

SOR1 Blank

SOR2 Source strength (particles/sec) when input by cards

AGE Time in years since isotopic composition was known

GRAMS Weight in grams of this source

Card S-7a Format (7E10.4) Source spectra (needed only if LETS $=0$ )

$\operatorname{KSP}(\operatorname{JMAXX}) \quad$ Source spectra by group

Card S-8 Format (7E10.0) Plutonium Isotopic Composition (not used if LETS $=0$ )

WTO(13) Isotopic weight fractions in the following order:

1. $P u-236$

2. $\mathrm{Pu}-238$

3. $\mathrm{Pu}-239$

4. $\mathrm{Pu}-240$

5. Pu-241

6. $\mathrm{Pu}-242$

7. Am-241

8. U-237 
9. U-232

10. Th-228

11. $\mathrm{Ra}-244$

12. $\mathrm{Pb}-212$ includes $\mathrm{Rn}-220$ and $\mathrm{Po}-216$

13. Bi-212 includes $\mathrm{T} 1-208$ and Po-212

Card S-9 Format (7E10.4) Spectrum weighting (needed if KWSP $=0$ or LETS $=0$ ). WSP (JMAXX) Source weighting by group.

The following input is read by the output routines and is not included on the restart tape so must be used with each restart.

Card $\theta-1$ Format (6I5)

NBSHSK

Number of batches to skip before starting output

processing. This could be used, for example to get

several generations away from the source distribution

when making a fission descendant calculation.

LCXST

0 - do not process or print cross sections or

reaction rates.

1 - print a11 reaction rates and cross sections

2 - print reaction rates only

3 - print region reaction rates only

LFXST

0 - print all fluxes

1 - suppress group flux ratio matrix

2 - suppress group and region flux ratio matrix

3 - print region flux ratio matrix only

NEBG

Number of output broad energy groups (Maximum of 4). 
Card $\theta-2$ Format $(6 E 12.0)$

VOL (NFMAX) Tally region volumes or volume ratios. Output is by tally region and is based on these volumes.

Card $\theta-3$ Format (14I5) Needed if NEBG $>0$.

JEBGT (NEBG) Lowest and highest numbered energy group contained

JEGB2 (NEBG) In each of four broad groups. The order would be $\operatorname{JEBG1}(1), \operatorname{JEBG} 2(1), \operatorname{JEBG1}(2), \ldots, \operatorname{JEBGI}(\mathrm{NEBG})$, JEBG2 (NEBG) .

Card $\theta-4$ Format (7E10.4) Not needed if LRAD is positive or 0 . FTDC (JMAXX) Conversion table for region averaged activities. LRAD sets of FTDC (JMAXX) needed. Flux to dose conversion factors go here now, but may be built in. (JMAXX is set on Card 4 and LRAD on Card 5.)

Card $\theta-5$ Format (14I5) Not used if LRAD is negative. IDRAD Control card for built in flux to dose conversion factors

1. Gamma flux to dose conversion factors

2. Neutron flux to dose conversion factors

3. Coupled neutron-gamma flux to dose conversion factors. 
APPENDIX B 


\section{PUSHLD}

PUSHLD is a point-kernel code written under the sponsorship of HEDL by H. H. Van Tuyl and J. N. Strode. This code was written specifically to calculate low energy gamma and $x$-ray dose rates through thin shields such as gloves.

This appendix contains an input description of the code only. A more detailed description of the code is available in the document HEDLTME 73-89, see reference 4 in the references section of the document. For this calculational system much of the input referred to in the document described above has been built into the code. Except for this, the codes described in the HEDL document and this document are the same. 
Card 1

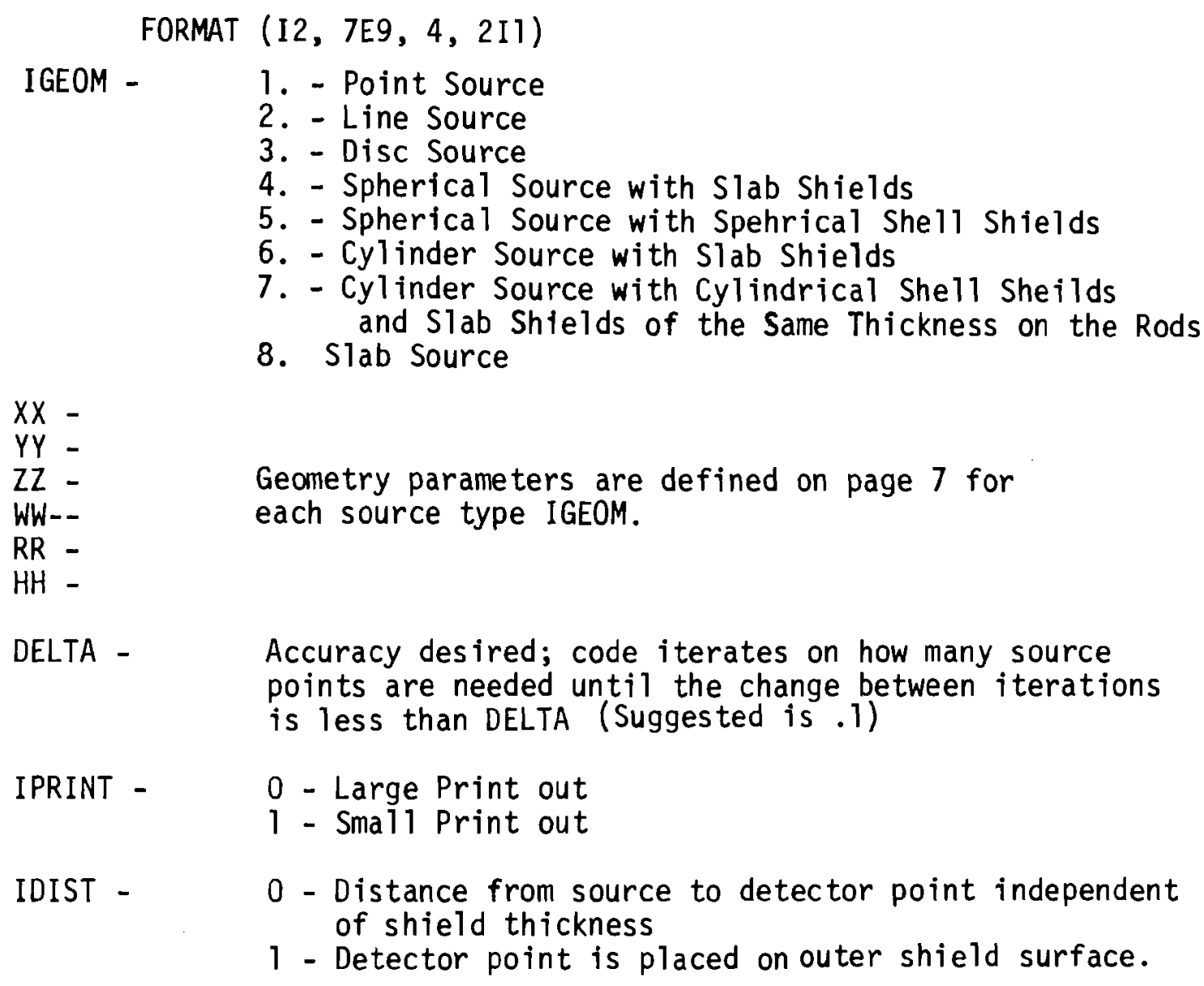
points are needed until the change between iterations is less than DELTA (Suggested is .1)

IPRINT - $\quad 0$ - Large Print out

1 - Sma11 Print out

IDIST - $\quad 0$ - Distance from source to detector point independent of shield thickness

1 - Detector point is placed on outer shield surface.

Card 2

FORMAT (E5.2)

WEIGHT - Weight of Pu in grams.

Card 3

FORMAT (I4 E5.2)

Elemental material compositions in the following order:

\begin{aligned} Card No. & Material \\ \hline 3 & Pu source \\ 4 & PVC \\ 5 & Lucite \\ 6 & Lead Glove \\ 7 & Steel \\ 8 & Lead \\ 9 & Lead glass \\ 10 & High density lead glove \\ 11 & Lead apron \\ 12 & Safety glass \\ 12 & Dun \end{aligned}


On each card are placed the elemental densities in the following order in grams/cc for each material above.

\begin{tabular}{cl} 
Order & Element \\
\cline { 3 - 3 } 1 & Pu \\
2 & Hydrogen \\
3 & Carbon \\
4 & Oxygen \\
5 & Clorine \\
6 & Iron \\
7 & Lead \\
8 & Calcium
\end{tabular}

Card 14

FORMAT (2I2)

NSHLD - Number of shields desired

NSHLD] - $\quad$ Number of shields remaining fixed

The code calculates dose rate through the fixed shields plus each of the other shields separately. One shield must remain fixed.

Card 15

FORMAT (14 E5.2)

T(NSHLD) - Thickness of each shield in centimeters. Fixed shields only must be ordered with increasing distance from the source. Does not include source.

Card 16

FORMAT (36I2)

IBLD(NCOMP) - Number designating the buildup factor to be used for each material in the order they are given on Cards $3-13$. 


\begin{tabular}{cl}
$\begin{array}{c}\text { Number } \\
\text { Designation }\end{array}$ & $\begin{array}{c}\text { Buildup Factor } \\
\text { Data }\end{array}$ \\
\cline { 2 - 2 } 1 & Water \\
2 & Aluminum \\
3 & Iron \\
4 & Tin \\
5 & Tungsten \\
6 & Lead \\
7 & Uranium
\end{tabular}

NCOMP is the number of materials specified on cards 3-13, now fixed at 11 .

Card 17

FORMAT (12A6)

TAG(NCOMP) - Alpha-numeric description of each shield material in the order given on Cards 3-13.

Card 18

FORMAT (36I2)

KSHLD (NSHLD) - Numerical index identifying the proper shield material for each thickness in the order given on card 15.

Numerical Index

1
2
3
4
5
6
7

8

9

10

11
Material

$\mathrm{Pu}$

PVC

Lucite Lead glove Steel Lead

Lead glass

High density lead glove Lead apron Safety Glass $\mathrm{PuO}_{2}$ 
Card 18

FORMAT (12A6)

TAGBLD(NBLD) - Alpha numeric description of buildup factors. Presently only those buildup factors in the order given below are available.

\author{
Water \\ A) uminum \\ Iron \\ Tin \\ Tungsten \\ Lead \\ Uranium
}

NBLD is the number of buildup factor sets available, now set at 7 .

Cards 20-32

FORMAT (A6-E9.2, 2I3, F4.0)

Cards describing source isotopes

SYMBOL(NUCL) - Alpha-numeric designation of the isotope (i.e. Pu-238)

HALF (NUCL) - Half-life in time units specified by

NUNIT(NUCL) - Numerical index designating time units of halflife

\begin{tabular}{c}
$\begin{array}{c}\text { Numerical } \\
\text { Index }\end{array}$ \\
\hline 1 \\
2 \\
3 \\
4 \\
5
\end{tabular}

Time

Units

Seconds

Minutes

Hours

Days

Years

NG(NUCL) - Number of energies with associated abundances built into code (not used).

AN(NUCL) - $\quad$ Atomic weight of isotope

NUCL is the number of source isotopes and is presently set at 13. The cards generally used are listed on the following page. The energies and associated abundance data for these isotopes are built into the code. 


\begin{tabular}{|c|c|c|c|c|c|}
\hline & Symbol & Half & NUNIT & $N G$ & AN \\
\hline 1 & $\mathrm{Pu}-238$ & 86.0 & 5 & 20 & 238 \\
\hline 2 & $\mathrm{Pu}-239$ & 24360 . & 5 & 56 & 239 \\
\hline 3 & $\mathrm{Pu}-240$ & 6760. & 5 & 16 & 240 \\
\hline 4 & $\mathrm{Pu}-241$ & 14.3 & 5 & 12 & 241 \\
\hline 5 & Pu-242 & $3.79 E+5$ & 5 & 12 & 242 \\
\hline 6 & Am-241 & 458. & 5 & 54 & 241 \\
\hline 7 & $\mathrm{U}-237$ & 6.75 & 4 & 29 & 237 \\
\hline 8 & $\mathrm{Pu}-236$ & 2.85 & 5 & 8 & 236 \\
\hline 9 & $U-232$ & 72. & 5 & 5 & 232 \\
\hline 10 & Th-228 & 1.913 & 5 & 5 & 228 \\
\hline 11 & RA-224 & 3.64 & 4 & 1 & 224 \\
\hline 12 & PB-212 & 10.64 & 3 & 6 & 212 \\
\hline 13 & BI -212 & 60.6 & 2 & 24 & 212 \\
\hline
\end{tabular}

Card 33

FORMAT (36I2)

NTM - $\quad$ Number of time steps

Card 34 .

FORMAT (12A6)

TIME(NTM) - Alpha numeric description of time step for print out.

Card 35.

FORMAT (14E5.2)

RTIME(NTM) - Time in ascending order for each time step in units determined by next card. 
Card 36

FORMAT (38I2)

ITUNIT(NTM) - Numerical index specifying time units for time steps specified on card 35.

\begin{tabular}{|c|c|}
\hline $\begin{array}{l}\text { Numerical } \\
\text { Index } \\
\end{array}$ & $\begin{array}{l}\text { Time } \\
\text { Units }\end{array}$ \\
\hline 1 & Seconds \\
\hline 2 & Minutes \\
\hline 3 & Hours \\
\hline $\begin{array}{l}4 \\
5\end{array}$ & Days \\
\hline
\end{tabular}

Card pairs 37 and 38.

\begin{abstract}
Card 37
FORMAT (14E5.2)

Weight percent of each isotope in the source in the same order as the isotopes listed on cards 20-32.
\end{abstract}

Card 38

FORMAT (12A6)

TITLE Card.

Any number of card pairs 37 and 38 are allowed. 
Definition of Geometry Parameters for Card 1

1. Point Source

$X X$ - Perpendicular distance* from the detector to a plane passing through the source and parallel to the slab shield.

$Y Y$ - Perpendicular distance from the source to the line defined by $x X$.

$X X$, WW, RR, HH - Not used.

2. Line Source

$X X$ - Perpendicular distance defined by the line from the detector point to the plane $P$ parallel to the shield and passing through the end of the line nearest the shield.

$Y Y$ - This distance is defined by a line in plane $P$ and parallel to the plane $Q$ formed by the source and its projection on the shield. The $Y Y$ entry is the distance from the 1 ine $X X$ perpendicular to the $p l a n e ~ Q$ where it contains the point defining the end of the source nearest the shield.

$Z Z$ - The perpendicular distance from the line YY to the $p l a n e Q$

WW - Similar to YY except that it applies to the end of the source farthest from the shield.

RR - Not used.

$H H$ - Not used.

3. Disc Source (No Self-Shielding)

General - Locate the center of the disc and the point on the edge nearest the shield. The center of the disc is the midpoint of a line $D$ formed by the intersection of the disc with a plane parallel to the shield and passing through the center of the disc.

$X X$ - The distance from the midpoint of the line $D$ (center of disc), to the detector point.

$Y Y$ - The perpendicular distance from the line $D$ to the 1 ine $X X$.

$Z Z$ - The distance along the line $D$ from the center of the disc to the $X Y$ plane.

*A11 distances are in centimeters. 
WW - The perpendicular distance to the line $x X$ from the point on the edge of the disc nearest the shield and contained in a line parallel to $D$.

$H H$ - Not used.

RR - Radius of the disc in centimeters.

4 and 5 . Spherical Source $\begin{aligned} \text { General - Similar to a point source, Either spherical shields } & \text { (5) or } \\ & \text { slab shields are possible } 4 \text { ? }\end{aligned}$

$X X$ - Perpendicular distance from the detector to a plane passing through the center of the spherical source and parallel to the slab shields. For spherical shell shields $X X$ is the distance from the center of the source to the detector point.

$Y Y$ - Perpendicular distance from the source center to the 1 ine defined by $X X$. Not needed for spherical shell shields.

$X X$ - Not used.

WW - Not used.

RR - Radius of spherical source in centimeters.

$H H$ - Not used.

6 and 7 . Cylindrical Source

General - Either slab shields ${ }^{(6)}$ or cylindrical shell shields with slab shields of the same thickness on the ends(7) are possible. The same type of coordinate system is used as with the line source.

$X X$ - Perpendicular distance defined by the line from the detector point to the plane $P$ parallel to the shield and passing through the end of the centerline nearest the shield. For cylindrical shell shields $X X$ is the perpendicular distance from the detector point to the centerline.

$Y Y$ - This distance is defined by a line in plane $P$ and parallel to the plane $Q$ formed by the centerline and $i$ ts projection on the shield. The $Y Y$ entry is the distance from the 1 ine $X X$ perpendicular to the plane $Q$ where it contains the point defining the end of the centerline nearest the shield. For cylindrical shell shields YY is the distance from the detector point to the plane of the nearest end of the cylinder. 
$Z Z$ - The perpendicular distance from the line $Y Y$ to the $P l a n e Q$.

WW - Similar to YY except that it applies to the end of the centerline farthest from the shield. For cylindrical shell shields WW is the distance from the detector point to the plane of the farthest end of the cylinder.

RR - Radius of the cylinder.

$\mathrm{HH}$ - Height of the cylinder.

8. Slab Source

$X X$ - The perpendicular distance from the detector point to the plane of the front of the slab source.

$Y Y$ - The perpendicular distance from the line $X X$ to the $P l a n e$ of the nearest side.

$Z Z$ - The perpendicular distance from the line $X X$ to the nearer plane of the top or bottom.

WW - Thickness of the slab source in the same direction as XX.

RR - Dimension of the slab in the same direction as $Y Y$.

$\mathrm{HH}$ - Dimension of the slab in the same direction as $\mathrm{ZZ}$. 
APPENDIX C 


\section{A Sample Simple Coupling Code*}

A coupling code is used for coupling $P_{N}$ neutron cross section sets, $P_{N}$ gamma-ray cross section sets, and multigroup secondary gamma-ray production cross sections. The neutron cross sections for ING neutron groups, the gamma-ray cross sections for IGG gamma groups, and the secondary gamma-ray production cross sections for ING neutron groups, IGG gamma groups are coupled into a $P_{N}$ cross section set of ING plus IGG groups. The neutron and gamma-ray cross section sets are input to the coupling code in ANISN structure. This is the same structure used in the BMC-MG code and explained in Appendix A, page 39. The secondary gamma-ray production cross sections must be input in the format which $\mathrm{POPOP} 4^{9}$ punches the cross sections. (This is the format used for cross sections available with the calculational system.)

A11 numerical input data are written in FIDO format. The characteristics of this subroutine as used in the BMC-MG code are described on pages 40-42 of Appendix $A$. The position of $\sigma_{\text {total }}$ and $\sigma_{g g}$ must be as shown on page 39 of Appendix $A$ for the neutron and gamma-ray $P_{N}$ cross section sets. A11 data sets presently in the library are for isotropically emitted gamma-rays and the coupling code therefore puts the secondary gamma-ray production cross sections in the coupled $P_{0}$ downscatter matrix and fills the corresponding matrix positions in the $P_{n}$ $(n>0)$ sets with zeros. The code includes an option to print and/or punch the MT coupled $P_{N}$ cross section sets. The first card of the punched output for each of the MT coupled sets will be the title card. The coupled sets are punched in FIDO format.

\footnotetext{
* Written by F. R. Mynatt and W. E. Ford, III. This appendix was taken
} directly from reference 12 with few changes. 
The variable dimensioning technique used in the Sample Simple Coupling Code requires an allocation of core in the blank common. Note that DUMY has been dimensioned by 40,000 (words). For a coupling problem to "fit" in the program, LAST must be less than or equal to the dimenston of DUMY, where

$$
\begin{aligned}
L A S T & =(I L G+I N G) \cdot(I N G+I G G)+I N G \cdot I G G(I P N+1) \\
& +I N G \cdot I G G+I G G \cdot I L G \cdot(I P N+1)+I N G \cdot I L N \cdot(I P N+1) \cdot
\end{aligned}
$$

The Sample Simple Coupling Code Input

1. $1 \$$ array - integer parameters in FIDO format (7 entries):

a. ING Number of neutron energy groups.

b. IGG Number of gamma energy groups.

c. ILN Length of the neutron cross section table.

d. ILG Length of the gamma-ray cross section table.

e. MT Number of $P_{N}$ coupled cross section sets expected.

f. IPO $0 / 1 / 2$ print/print and punch/punch the coupled $P_{N}$ cross section set(s).

g. IPN Order of scatter of the cross section sets $\left(N\right.$ in $\left.P_{N}\right)$.

End the $1 \$$ array with a $T$. 
The following data are repeated MT times:

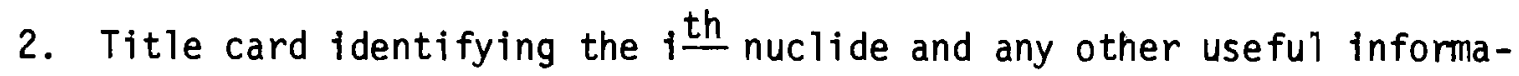
tion (format 20A4). If IPO is greater than zero, this title card is punched as the first card of the punched $P_{N}$ coupled cross section set.

3. $2^{\star}$ array - floating point parameters in FIDO format (ING $\cdot$ ILN $\cdot($ IPN+1) entries):

The $P_{N}$ neutron cross section sets for the $i \frac{\text { th }}{\text { nuclide. The } P_{0}}$ set is entered first, then the $P_{1}$ set, etc.

4. $3^{*}$ array - floating point parameters in FIDO format (IGG $\cdot$ ILG. (IPN+1) entries):

The $P_{N}$ gamma-ray cross section sets for the $i$ th nuclide. The $P_{0}$ set is entered first, then the $P_{1}$ set, etc.

5. $4^{\star}$ array - floating point parameters in FIDO format (ING.IGG entries):

The $P_{0}$ multigroup secondary gamma-ray production cross sections for the $i$ th nuclide. The cross sections for the highest neutron group are entered first - one value for each gamma group (highest ganma group first).

End the $4^{*}$ array with a $T$.

This concludes the input for the $i \frac{\text { th }}{\text { nuclide. If } i<M T, ~ r e t u r n ~ t o ~} 2$ above and enter the title card, $2^{\star}, 3^{\star}$, and $4^{*}$ arrays for the $i+1$ th nuclide. If $i=M T$, this concludes the input for the coupling code. 
No. of

Copies

\section{OFFSITE}

1

ERDA Chicago Patent Group

Argonne, Illinots 60439

A. A. Churm

3

ERDA Division of Military Applications Washington, DC 20545

D. D. Davis

F. X. Murray

T. R. Clark

27

2

Rockwe11 International

Rocky Flats Plant

P.0. Box 464

Golden, Colorado 80401

D. Coonfield

E. A. Putzier

1 Union Carbide Corporation

Nuclear Division - AECOP

Federal Office Building

P.0. Box S

Oak Ridge, Tennessee 37830

J. M. Morrison

2 University of California

Lawrence Livermore Laboratory

P.0. Box 808

Livermore, Californta 94550

D. L. Goldman

J. L. Robbins 
No. of

Copies

ONSITE

1

ERDA Richland Operations Office

B. J. Melton/P. F. X. Dunigan, Jr.

4

Atlantic Richfield Hanford Company

R. D. Carter

D. E. Larson

A. E. Smith

R. W. Szempruch

4

Hanford Engineering Development Laboratory

D. R. Marr-

R. C. Smith

J. N. Strode

W. L. Bunch

29 Battelle-Northwest

L. W. Brackenbush

C. L. Brown

N. E. Carter

L. G. Faust

R. R. King

R. A. Libby

A. M. Platt

R. W. Stewart

D. H. Thomsen

H. H. Van Tuyl

M. G. Zimmerman (15)

Techntcal Information Library (3)

Technical Publications 